\title{
Molecularly Imprinted Electroimpedance Sensor for Detection of 8-isoprostane in Exhaled Breath Condensate
}

\author{
by
}

Bruno Gamero

A thesis submitted to the Faculty of Graduate and Postdoctoral Affairs in partial fulfillment of the requirements for the degree of

\author{
Master of Applied Science \\ in \\ Electrical and Computer Engineering
}

Carleton University

Ottawa, Ontario

(C) 2021, Bruno Gamero 


\begin{abstract}
Development of low-cost, rapid response time capacitive sensors have a valuable role in the creation of point-of-care systems. A novel approach in the materials and application of Molecularly Imprinted Polymers was investigated for the detection of 8isoprostane in exhaled breath condensate. The detection method is based on the quantifiable capacitance change that occurs between two electrodes as the target molecule binds on the MIPs surface, which is detected through electrochemical impedance spectroscopy. This work focuses on the use of a generic polymer material for the sensing layer, as opposed to a traditional synthesized polymer material. PVA-SbQ was spun onto a custom IDE and then imprinted to detect 8-isoprostance. With aerosolized samples, the sensor was proven to detect a physiologically relevant concentration of 1 to $100 \mathrm{pg} / \mathrm{mL}$. A fully integrated multiplexed system was then developed for point-of-care health monitoring.
\end{abstract}




\section{Co-Authorships}

The work described in the report was developed in the Organic Sensor and Devices Laboratory at Carleton University in 2019-2021. The work described has led to a journal (published) and a conference paper (submitted). Further work was done on a project investigating a fluorinated graphene oxide (FGO) based chemiresistive gas sensor, which led to the co-authorship of a published conference paper.

The reported Capacitive MIPs sensos was able to quantify concentrations of 8isoprostane in aqueous and aerosolized samples with high sensitivity and selectivity. A complete impedimetric analysis of the sensor output data is used to analyze the sensor response with a custom analysis algorithm. The paper focuses on the synthesis of the sensor, with an emphasis on the use of a generic polymer imprinting material to simplify the fabrication of the sensor. The properties of the MIPs surface are analyzed and comparable molecules to 8 -isoprostane are used to quantify the sensitivity and specificity of the sensor. The second in progress paper has been submitted for a conference presentation, with a focus on sensor integration.

The publications on the MIPs capacitive sensor, along with the work presented in this thesis, was conducted under the supervision of Prof. Ravi Prakash, and in collaboration with research associate Dr. Siziwe Bebe in the Organic Sensors and Devices Laboratory (OSDL) at Carleton University. The electrode patterning was performed by Mr. Rob Vandusen of the Carleton University Microfabrication team. AFM imaging was performed with the assistance of Mr. Rob Vandusen and Dr. Siziwe Bebe. The FTIR measurements were provided by Dr. Siziwe Bebe, and SEM imaging was performed at NRC Ottawa. The deposition and imprinting techniques were developed and performed with the assistance of 
Dr. Siziwe Bebe. The test setup, data acquisition, modeling, data analysis, and PCB design were performed solely by the author.

The FGO chemiresistive gas sensor project, referenced as $\mathrm{C}$, was performed in collaboration with the same group and the main author, Mr. Ivan Amor. For this report, the author assisted in the development of the readout circuitry, measurements, and data analysis of initial tests. This conference paper describes the sensors' ability to detect ammonia and acetone at ultra-low concentrations in gaseous forms. The paper provides an analysis of the functionalization process of the graphene-oxide, the readout system, and the usability of the sensor for point-of-care health monitoring.

Authorship References:

A. Bruno Gamero, Siziwe Bebe, Ravi Prakash, "Molecularly Imprinted Electroimpedance Sensor for Detection of 8-isoprostane in Exhaled Breath Condensate," in IEEE Sensors Letters, Vol. 5, No 9, DOI: 10.1109/LSENS.2021.3103433, pp. 1-4, September 2021

B. B. Gamero, S. Bebe and R. Prakash, "Integrated Molecularly Imprinted Electroimpedance Sensor for Detection of 8-isoprostane in Exhaled Breath Condensate," in Micro and Nanotechnology in Medicine conference, (in progress)

C. Ivan Amor, Bruno Gamero, Siziwe Bebe, Ravi Prakash, "Hydrothermally Fluorinated Graphene Oxide Chemiresistive Sensor for Detecting NH3 and Acetone under Atmospheric Conditions," In Proceedings of the 14th International Joint Conference on Biomedical Engineering Systems and Technologies, Vol. 1 BIODEVICES, ISBN 978-989-758-490-9, pp. 99-106, February 2021 


\section{Acknowledgements}

I would like to thank my research supervisor, Prof. Ravi Prakash, for all his support and guidance through this project. His enthusiasm towards biosensors and his dedication towards research has been a constant inspiration for me throughout the duration of this research project. He was always available for discussions about project activates, providing suggestions for ways of incorporating new methods and technologies, and discussing life goals. This work was only made possible thanks to his assistance and belief in my work,

I would also like to thank the research team at the Carleton OSDL lab, especially Dr. Siziwe Bebe. Her chemical expertise and advice were instrumental in the development of the project, especially for the polymer fabrication process. Her kind words were always a joy to have in the lab. I would also like to thank Mr. Ivan Amor, Ms. Roslyn Massey, and Ms. Mounia Chakik for their friendship and advice throughout the project.

From the Carleton University Microfabrication Lab, I would like to thank Mr. Rob Vandusen for doing the electrode patterning, as well for all his support with performing the AFM and FTIR analysis for the project. I would also like to thank NRC for performing SEM imaging on the sensor surface, as well as NSERC for the assistance through the NSERC Discovery grant.

Finally, I would thank all my friends and family, for their unconditional love and support throughout this process. Their constant support has helped me remain motivated and push myself to perform at my best. 


\section{Table of Contents}

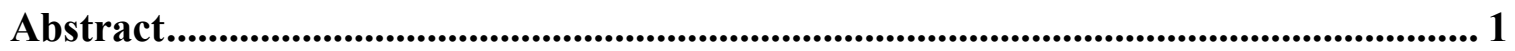

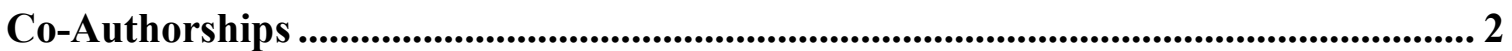

Acknowledgements ................................................................................................................. 4

Table of Contents ......................................................................................................................... 5

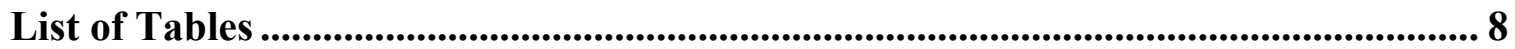

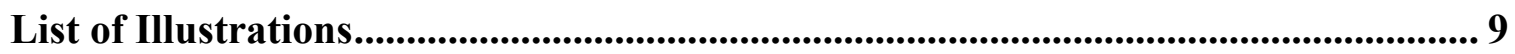

List of Abbreviations and Symbol .......................................................................................... 12

Chapter 1: Introduction ........................................................................................................ 13

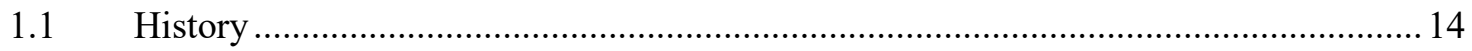

1.2 Introduction to Molecularly Imprinted Polymers ...........................................................16

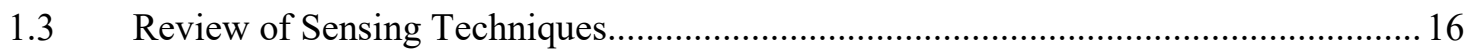

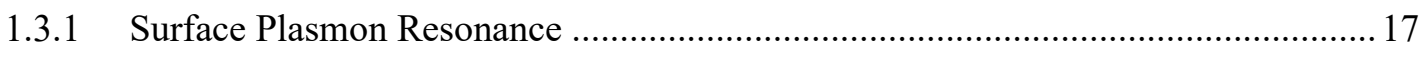

1.3.2 Resonance Light Scattering .................................................................................. 19

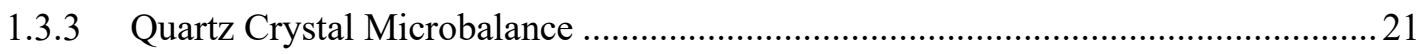

1.3.4 Surface Wave Acoustic .........................................................................................22

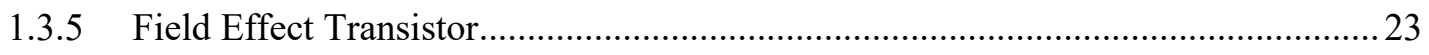

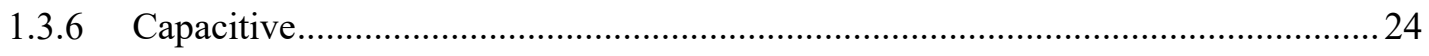

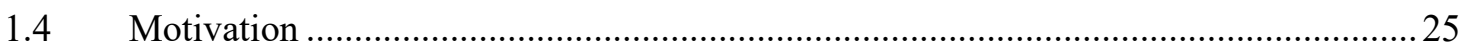

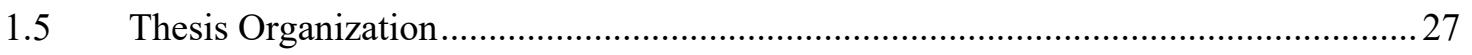

Chapter 2: Literature Review............................................................................................. 28

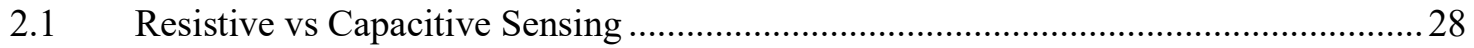

2.2 Materials for Molecularly Imprinted Polymers …………………………………….... 32

2.3 Molecularly Imprinted Polymers Fabrication Methods............................................... 33 


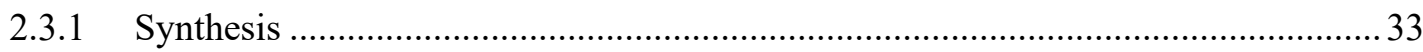

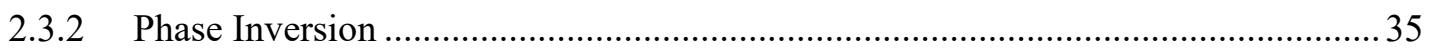

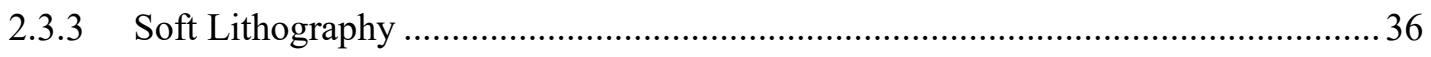

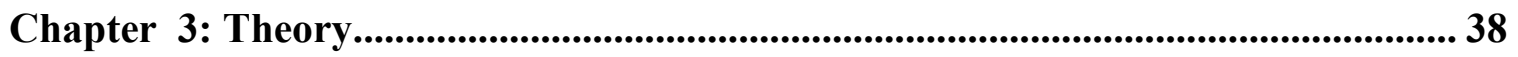

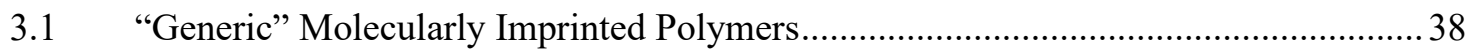

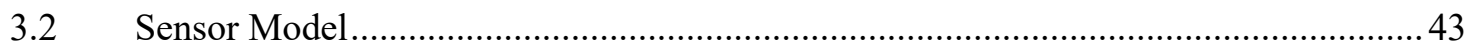

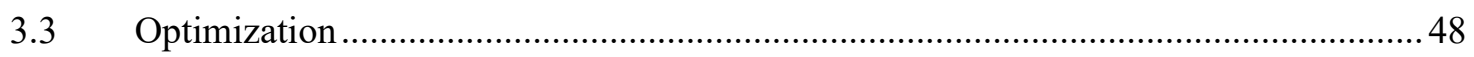

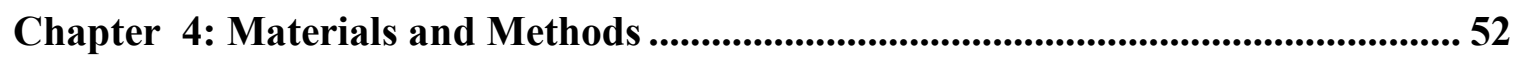

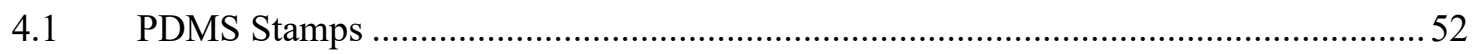

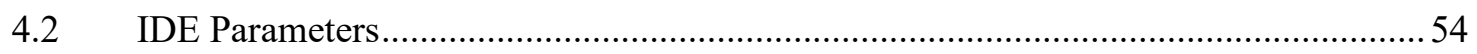

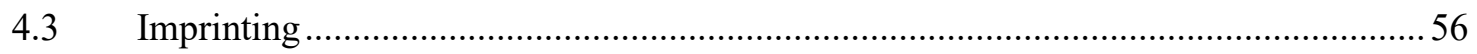

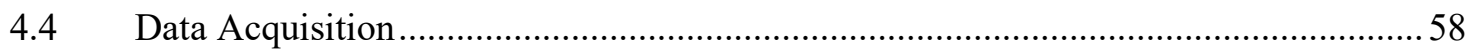

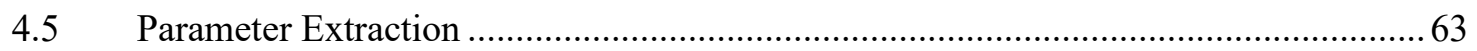

Chapter 5: Experimental Results and Discussion................................................... 69

5.1 Physical and Chemical Characterization of the MIP sensing Layer............................69

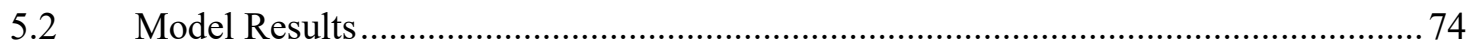

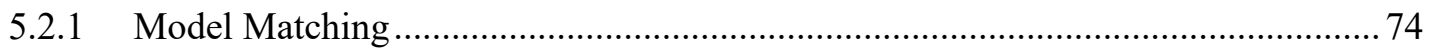

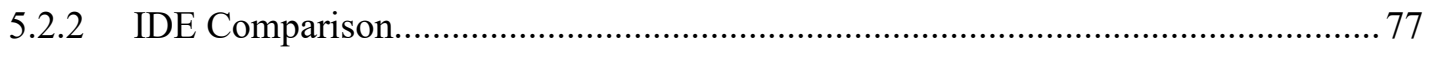

5.3 Sensor Performance in Aqueous and Aerosolized Sample Testing............................. 79

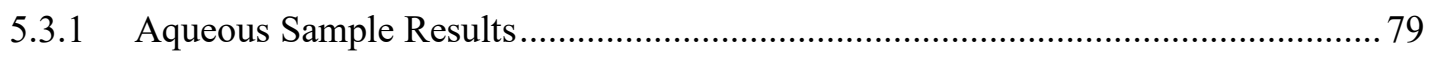

5.3.2 Cross-sensitivity Validation of the MIP-EIS Sensor........................................... 81

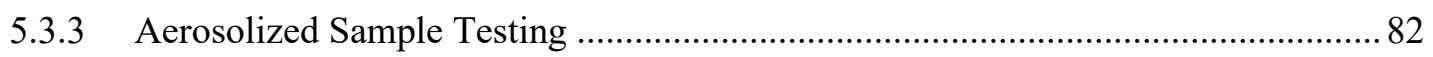

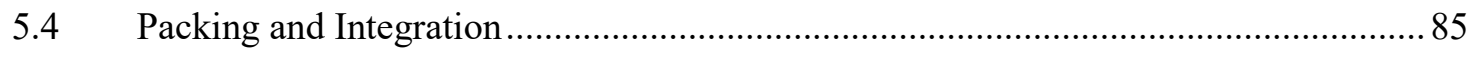

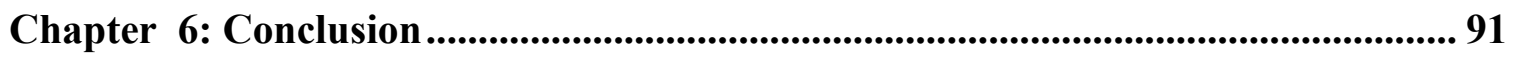

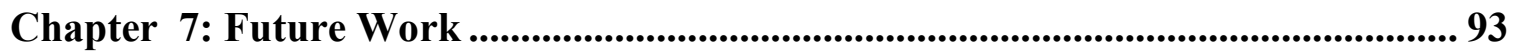


Appendices

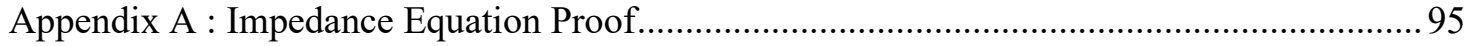

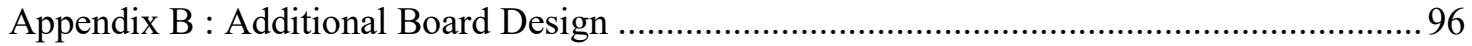

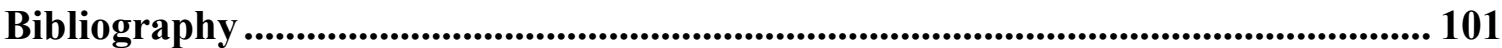




\section{List of Tables}

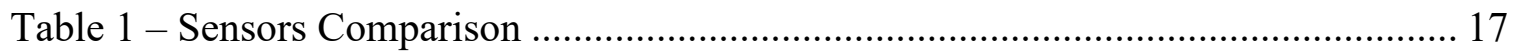

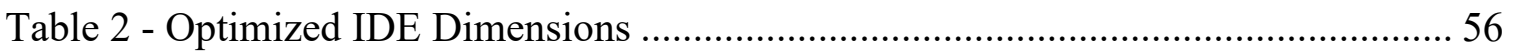

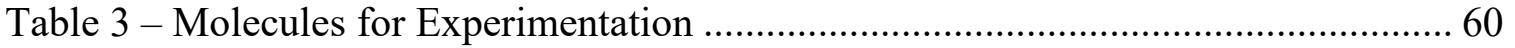




\section{List of Illustrations}

Figure 1 - Basic Sensor Architecture …………………..................................... 14

Figure 2 - SPR Sensor Components (reproduced from [21]) …………........................ 18

Figure 3 - RLS Sensor Design (reproduced from [100]) ………….............................. 20

Figure 4 - QCM sensor and equivalent circuit model (reproduced from [29]) ................ 21

Figure 5 - SAW sensor design and wave propagation (reproduced from [34]) …........... 23

Figure 6 - CHEMFET sensor Design (reproduced from [99]) ………………………..... 24

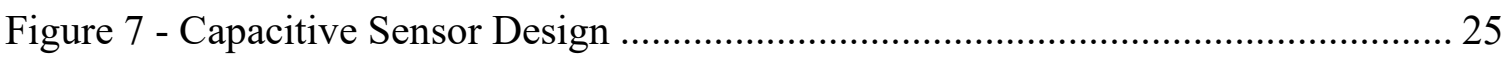

Figure 8 - (a) Example of a Multi-Layer Resistive sensor for $\mathrm{NH}_{3}$ Sensing (reproduced from [47]) (b) Basic IDE structure with Important Dimensions..................................... 30

Figure 9 - MIPs synthesis technique (reproduced from [53]) ........................................ 35

Figure 10 - MIPs Phase Inversion technique (reproduced from [54]) ……………......... 36

Figure 11 - MIPs Soft Lithography Technique ……………………………………....... 37

Figure 12 - Molecular structure of: (a) PVA-SbQ (reproduced from [59]) (b) 8isoprostane (reproduced from [60]) (c) PDMS (reproduced from [61]) ........................... 40

Figure 13 - PVA-SbQ crosslinking structural change (reproduced from [68]) ……......... 41 Figure 14 - (a) Full Equivalent Circuit model (b) Simplified Equivalent Circuit Model 44 Figure 15 - Impedance Magnitude and Phase from model (reproduced from [71]) ......... 47 Figure 16 - (a) Patterned PDMS Stamps (b) AFM of patterned PDMS Stamp ............... 53 Figure 17 - Optimized IDE Layouts used for Aerosolized Sample Testing ………….... 55 Figure 18 - (a) Protected IDE with deposited PVA-SbQ (b) IDE spun, tape removed, and stamp placed (c) IDE clamped with glass slide for UV exposure (d) Final imprinted

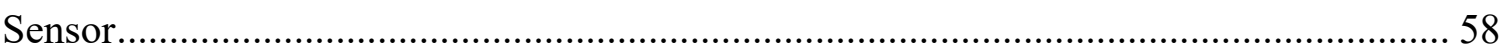


Figure 19 - Aerosolized Sample Setup ………………….......................................... 59

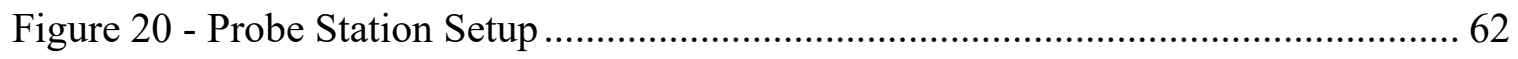

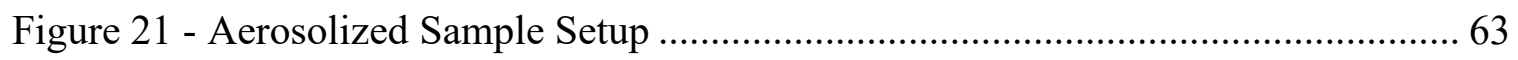

Figure 22 - Representative Nyquist plot of impedance ............................................... 65

Figure 23 - Measured impedance example of no clear corner ......................................... 66

Figure 24 - Comparison of Stamps 1-4 (a-d) using AFM .............................................. 69

Figure 25 - (a) AFM of Stamp 1 surface roughness (b) Image of Stamps 0-4................ 70

Figure 26 - Stamp Reusability, AFM image of Stamp 4 after creating the stamp and after

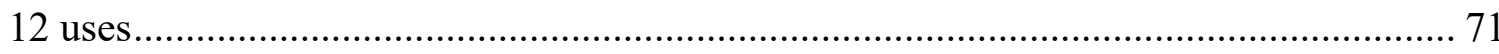

Figure 27 - (a) Cross section of sensor (b) Close up of polymer layer (c) Stamp 1 surface

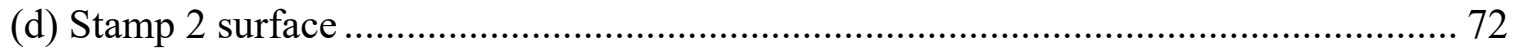

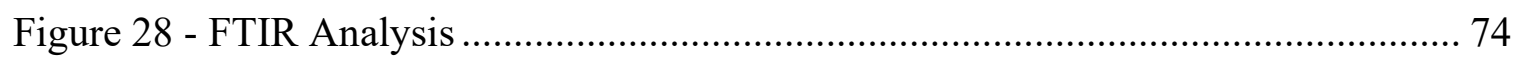

Figure 29 - Ideal Impedance Model vs Measurement with two approaches (a) Nyquist

Plot (b) Impedance Magnitude Plot (c) Impedance Phase Plot .......................................... 75

Figure 30 - Non ideal Impedance Model vs Measurement Magnitude and Phase ........... 77

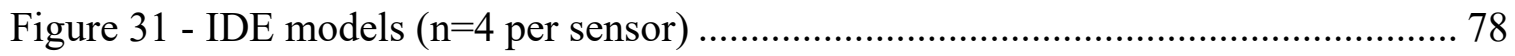

Figure 32 - Comparison of Sensor Sensitivity at $100 \mathrm{pg} / \mathrm{mL}$ ( $\mathrm{n}=6$ per sensor) ................ 78

Figure 33 - Sensor Response Time for Aqueous Samples............................................ 80

Figure 34 - Stamp 1 and 2 Sensor Comparison ( $\mathrm{n}=7$ per sensor) ………....................... 81

Figure 35 - Aqueous Solution Selectivity ( $\mathrm{n}=7$ per sensor).......................................... 82

Figure 36 - Aerosolizes Sensor Time response............................................................. 84

Figure 37 - Aerosolized Sensor CG Response (n=6 per sensor) ……………………..... 84

Figure 38 - Aerosolized Sensor CDL Response ( $\mathrm{n}=6$ per sensor) .................................. 85 
Figure 39 - Integrated PCB-Sensor Assembly .................................................... 86

Figure 40 - Sample EIS measurement from integrated board................................... 87

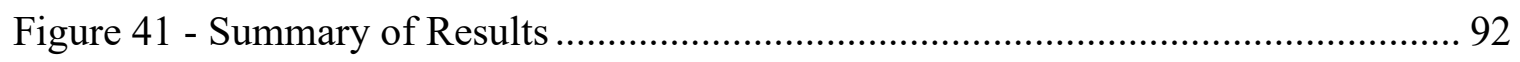

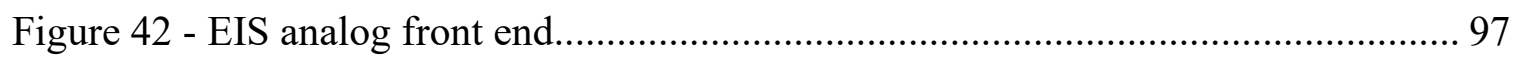

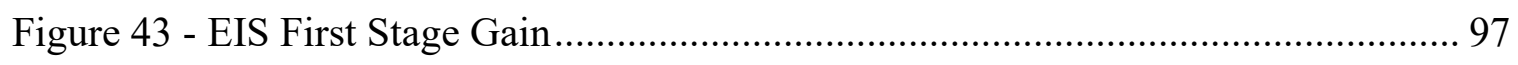

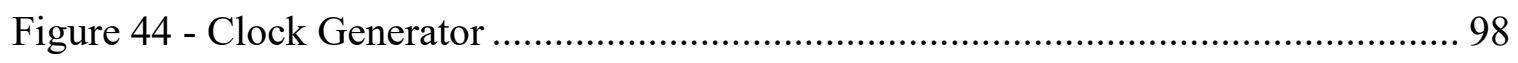

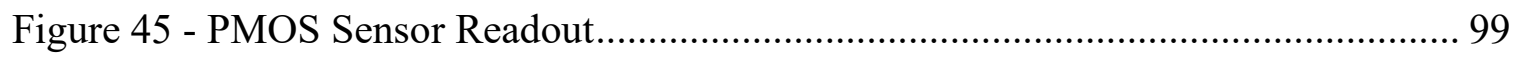

Figure 46 - Board Layout (without copper pours) ................................................. 100 
List of Abbreviations and Symbol

\begin{tabular}{|c|c|c|c|}
\hline Abbreviation & Meaning & Symbol & Meaning \\
\hline 8-iso/8-isoprostane & 8-iso Prostaglandin F2 $\alpha$ & A & IDE-Dielectric interface area \\
\hline ADC & Analog to Digital Converter & $\alpha$ & CPE phase \\
\hline AFM & Atomic Force Microscope & $\mathrm{C}$ & Speed of Light \\
\hline CHEMFET & Chemical FET & $C_{\alpha}$ & CPE unit \\
\hline CPE & Constant Phase Element & $C_{C}$ & Mass sensitivity constant \\
\hline DAC & Digital to Analog Converter & $\mathrm{C}_{\mathrm{DL}}$ & Double layer capacitance \\
\hline DI & Deionized & $\mathrm{C}_{\mathrm{DL}, \text { surface }}$ & Double layer surface constant \\
\hline ECM & Equivalent Circuit Model & $\mathrm{C}_{\mathrm{G}}$ & Geometry Capacitance \\
\hline EIS & Electroimpedance Spectroscopy & $\mathrm{CP}_{\mathrm{P}}$ & Parasitic Capacitance \\
\hline EM & Electromagnetic & $\epsilon_{0}$ & Dielectric constant \\
\hline FET & Field Effect Transistor & $\epsilon_{r}$ & Relative dielectric \\
\hline FGO & Fluorinated Graphene Oxide & $\epsilon_{s}$ & $\begin{array}{l}\text { Coating-Sample equivalent } \\
\text { dielectric }\end{array}$ \\
\hline FTIR & Fourier Transform Infrared & $F$ & Frequency in hertz \\
\hline GO & Graphene Oxide & $f_{\mathrm{HIGH}}$ & ECM high corner frequency \\
\hline IDE & Interdigitated Electrode & $f_{\text {LOW }}$ & ECM low corner frequency \\
\hline IDT & Interdigitated Transducer & $\mathrm{Hz}$ & Hertz \\
\hline LOD & Limit of detection & $I$ & current \\
\hline MEMS & Microelectrochemical systems & $\mathrm{I}_{\mathrm{RLS}}$ & Resonant Light Scattering Intensity \\
\hline MIP & Molecularly Imprinted Polymer & $\mathrm{J}$ & Imaginary component \\
\hline NRC & National Research Council & $\mathrm{K}$ & Wavevector \\
\hline NSERC & $\begin{array}{l}\text { Natural Sciences and Engineering } \\
\text { Research Council of Canada }\end{array}$ & $K_{\text {CELL }}$ & Cell constant \\
\hline OEGFET & Organic electrolyte-gated FET & $\mathrm{k}_{\mathrm{sp}}$ & Wavevector surface plasmon \\
\hline OpAmp & Operational Amplifier & $\mathrm{L}$ & IDE finger length \\
\hline OSDL & Organic Sensors and Devices Laboratory & $\mathrm{N}$ & IDE finger number \\
\hline PCB & Printed Circuit Board & $\Omega$ & Resistance \\
\hline PDMS & Polydimethylsiloxane & $\mathrm{R}_{\mathrm{C}}$ & Contact Resistance \\
\hline PEDOT:PSS & $\begin{array}{l}\text { Poly(3,4-ethylenedioxythiophene) } \\
\text { Polystyrene Sulfonate }\end{array}$ & $\mathrm{R}_{\mathrm{S}}$ & Coating Resistance \\
\hline PMMA & Polymethyl Methacrylate & $\mathrm{S}$ & IDE finger spacing \\
\hline PSI & Pounds per square Inch & $\mathrm{V}$ & Volts \\
\hline PVA & Polyvinyl Alcohol & $\mathrm{W}$ & IDE finger width \\
\hline PVA-SbQ & $\begin{array}{l}\text { Poly(Vinyl Alcohol), N-Methyl-4(4'- } \\
\text { formylstyryl)Pyridinium Methosulfate } \\
\text { Acetal }\end{array}$ & $\omega$ & Frequency in radians \\
\hline QCM & Quartz Crystal Microbalance & $\mathrm{Z}_{\mathrm{CPE}}$ & Constant Phase element impedance \\
\hline RLS & Resonance Light Scattering & $\sigma_{\mathrm{S}}$ & coating conductivity \\
\hline RPM & Rotation Per Minute & & \\
\hline SAW & Surface Wave Acoustic & & \\
\hline SEM & Scanning Electron Microscope & & \\
\hline SPR & Surface Plasmon Resonance & & \\
\hline $\mathbf{U V}$ & Ultraviolet & & \\
\hline VOC & Volatile Organic Compound & & \\
\hline
\end{tabular}




\section{Chapter 1: Introduction}

The analysis of the contents of exhaled breath condensate has been well understood to be a valuable marker of health, especially in the detection of pulmonary diseases [1]. Exhaled breath sensors can provide a rapid analysis for certain analytes in a patient's breath, which can be a valuable method for early detection of certain diseases [2]. These sensors can employ a wide variety of sensing mechanisms, whether label-based or labelfree, and both types can utilize a wide range of technologies to generate a response [3]. One such method of label-free detection is through the use of thin-film capacitive sensors, which consists of a high dielectric material deposited over an electrode structure. The deposited material is then modified through either a chemical or physical process to interact with a certain target analyte, leading a change in the effective dielectric seen by the electrode structure when exposed to said analyte. As the analyte binds to the sensitive coating material, the change in equivalent dielectric leads a change in the capacitance, which is used to determine the analyte concentration [4].

These sensors have been used for a wide variety of applications, with many different methods to functionalize the deposited material. One method of functionalization is to use Molecularly Imprinted Polymers (MIPs), where the sensor coating is a polymer that is imprinted at a molecular level to create binding sites that are sensitive to a specific target [5]. This technology has been demonstrated to function with analytes ranging from small molecules in the nm range to complex biological samples such as viruses in the $\mu \mathrm{m}$ range [6]. The advantage of these sensors is that they can achieve performance comparable to antibody-based sensors, which have the same sensing mechanism, while having improved durability and shelf life. MIPs sensor have seen use in point-of-care applications 
such as gas sensing of volatile organic compounds (VOCs) [7] and bacteria sensing in aqueous solutions [8]. The basic functionality of these sensors is demonstrated in Figure 1, showing that target molecules bind to the sensing surface which can be detected by a variety of methods and turned into a measurable electrical signal.

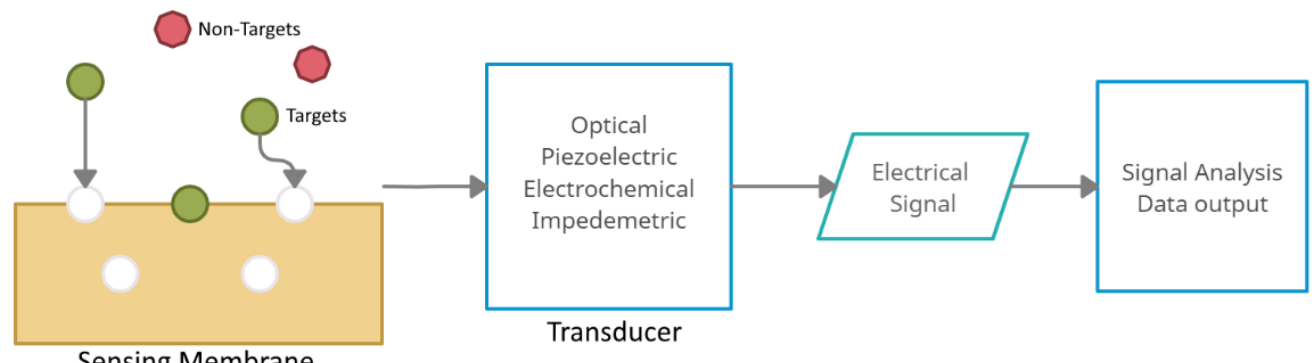

Figure 1 - Basic Sensor Architecture

The polymer material is carefully curated for most applications, with considerations towards the cross-linking properties, the dielectric, the ability to bond the substrate, and its susceptibility to damage when washing out bound molecules. The method of imprinting must be carefully considered and optimized for different applications.

\subsection{History}

The study of molecularly imprinted polymers dates back to the early 1930's with a paper by M.V. Polyakov analyzing the effects of different solvents on a silica gel pore structure, demonstrating that the absorption and structure were modified depending on the solvent [9]. Although not yet referred to as "Molecularly Imprinted Polymers," further research was conducted over the next several years regarding the properties of silica gel and the tailoring of its absorption. In 1949 it was predicted that polymers with tailored absorptions would soon be available as the understanding of molecular structures improved [10], and that same year Frank H. Dickey published a method for the preparation of adsorptions with affinities to selected substances [11]. Similar to how the imprinted process 
is done today, this method involved having the desired substance within the adsorbent while its structure is formed, creating binding sites that would allow the desired substance to bind to the adsorbent. Over the next 35 years as the understanding of the imprinted process improved, new methods of adsorbent production were developed, mostly focusing on silica gel as the adsorbent material [12]. The first mention of "molecular imprinting" does not appear in research papers until 1984, in a paper by Günter Wulff simply titled "Molecular Imprinting," focusing on the use of covalent bonding in imprinting [13]. During this period, research was focused on the use of covalent bonding or noncovalent interactions in the creation of the imprints, with the covalent binding creating more homogenous and more sensitive MIPs. The first reported MIPs sensor was presented in the early 1990s, and since then the research has expanded into developing new MIPs production techniques, analysis and design of MIPs materials, and MIPs based sensors.

To this the day commercialization of MIPs has been progressing at a slow rate [14]. Due the nature of the MIPs imprinting methods, the distribution of binding sites and their affinities is difficult to keep constant between batches, leading to a difficulty in ensuring predictable and constant sensor performance. While no MIPs based sensor has been publicly commercialized, recent reports of commercial-ready sensors indicate that is likely these sensors will become commercial products within the next few years. Currently, only a few companies exist that work with MIPs technologies, the fist being MIP Technologies in Sweden, founded in 2000 and later acquired by Biotage AB. These companies focus on the synthesis of the molecularly imprinted polymers, and not on their uses for sensors. 


\subsection{Introduction to Molecularly Imprinted Polymers}

As the name suggests, a molecularly imprinted polymer is a polymer that has been imprinted at a molecular level in order to have nanocavities that act as binding sites towards a template. Also referred to as "plastic antibodies," these polymers work in the same way, as the high affinity binding sites act as receptors, only binding to the molecular structure to which it has been imprinted. Although most molecularly imprinted polymers do not achieve the same sensitivity and selectivity compared to antibody-based sensors, MIPs sensors can achieve comparable performance while having a much longer shelf due to its synthetic nature. Theses sensors are also much more robust, being much less sensitive to environmental factors such as temperature, humidity, and acidity. The characteristics of the MIPs sensor is dictated by the imprinting process, the selection and ratio of materials in the imprinting process, and the sensor design. The advantages of the MIPs sensors over antibody sensors and its quick label free sensing have led this technology to be used in a wide variety of sensor types.

\subsection{Review of Sensing Techniques}

Along with a wide variety of thin-film functionalization methods, a wide variety of sensors have been developed using the thin-film technique, and some of the more common methods are highlighted in the following section. These methods can be classified into three main categories: optical, piezoelectric, and impedimetric. Although any thin film sensing layer can be used in these sensors, the examples provided are all based on MIPs based sensing. Most of these sensors have demonstrated detection of ultra low concentrations. The following sections briefly describe the most common types of sensors 
that utilize the different sensing methods and their uses. Table 1 provides a summary of the sample types commonly used for each sensor, as well as a comparison of their respective limits of detection based on recent work.

Table 1 - Sensors Comparison

\begin{tabular}{|l|l|l|}
\hline Sensor Type & Typical Applications & Limit of Detection \\
\hline Surface Plasmon Resonance & Solution & $\sim 0.1 \mathrm{pg} / \mathrm{mL}[15]$ \\
\hline Resonance Light Scattering & Solution & $\sim 1.1 \mathrm{pmol} / \mathrm{mL}[16]$ \\
\hline Quartz Crystal Microbalance & Gas & $\sim 0.3 \mu \mathrm{M} \mathrm{[17]}$ \\
\hline Surface Acoustic Wave & Gas & $\sim 0.4 \mathrm{nM} \mathrm{[18]}$ \\
\hline Field-Effect Transistor & Solution & $\sim 0.1 \mathrm{pM} \mathrm{[19]}$ \\
\hline Capacitive & Gas, Solution & $<1 \mathrm{pM} \mathrm{[20]}$ \\
\hline
\end{tabular}

\subsubsection{Surface Plasmon Resonance}

The MIPs-Surface Plasmon Resonance (SPR) is optical sensor that relies of electromagnetic waves propagating along the surface of a thin metal or nanoparticle layer [21]. The surface plasmons can be described as the cumulative oscillation of conduction electrons in metallic nanoparticles close to the nanoparticle - dielectric interface and is characterized by the enhancement of the electromagnetic field, the propagation length, and the penetration depth [22]. The resonance angle is the angle the intensity of the electric field at the interface between the nanoparticles and dielectric is larger on one side of the nanoparticles compared to the other side. This is caused by the smaller complex permittivity of the dielectric compared to the metal nanoparticle, and this phenomenon is called enhancement. The propagation length is a characteristic of how far the EM wave 
propagates parallel to the plane of propagation (at the nanoparticle - dielectric interface) and will depend on the dielectric value. As described by Abbas et al. [23], for an electromagnetic wave in a medium, the wavevector is given as:

$$
k=\frac{\omega}{c} \sqrt{\epsilon_{i}}
$$

In the equation above, $\omega$ is the angular frequency, $\mathrm{c}$ is the speed of light, and $\epsilon_{i}$ is the relative permittivity of the medium. Since the EM wave occurs at the interface of the two mediums, both dielectrics are part of the effective dielectric, which gives an in-plane wavevector of:

$$
k_{S P}=\frac{\omega}{c} \sqrt{\frac{\epsilon_{m} \epsilon_{d}}{\epsilon_{m}+\epsilon_{d}}}
$$

The SP refers to the fact that this is the surface plasmon as the wavevector radiates along the interface surface, whereas $\epsilon_{m}$ and $\epsilon_{d}$ are the dielectrics of the metal and dielectric sensing layer. The generated EM wave also has a perpendicular component, and the penetration depth characterizes how far this wave propagates perpendicular to the surface, which is a function of the metal and dielectric sensing layers dielectric constants. Gold nanoparticles are commonly embedded into a molecularly imprinted polymer layer, and as targets bind to the MIPs layer the effective dielectric will be affected. As shown in

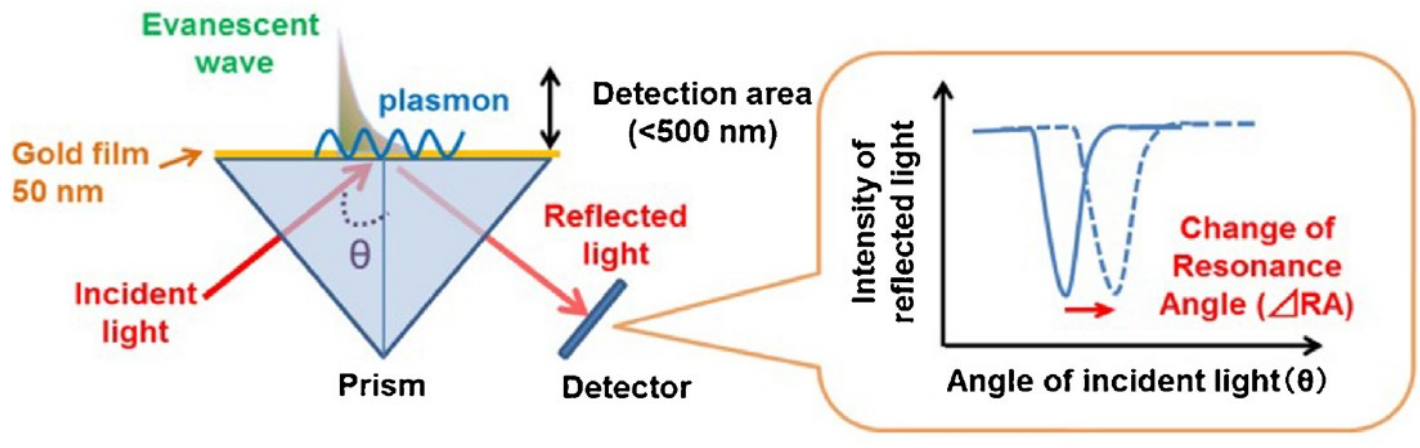

Figure 2 - SPR Sensor Components (reproduced from [21]) 
equations 1.1 and 1.2 , this change in dielectric will change the surface plasmon properties, which is detected and correlated to the concentration of analyte.

These sensors require a light source to generate polarized light that passes through a prism and hits the sensing surface, as shown in Figure 2. The light leaks in electric field called the evanescent wave on the lower refractive index medium. Some of the photons from applied light will excite the electrons at the nanoparticle - dielectric interface causing them to start oscillating and generating the surface plasmon. Another portion of the light is reflected through the prism to an optical detection unit. The refractive index of the light will depend on the surface plasmon, and the reflected light intensity, angle, and wavelength are measured and characterized, and is proportional to the mass of the bound molecules. Due to enhancement depending on the nanoparticle size, a well-designed sensor can achieve subwavelength sensitivity, meaning that wavelengths shorter than surface plasmon frequency can be analyzed. Akgönüllü et al. have recently demonstrated a MIPs based SPR sensor utilizing gold particles for the detection of Aflatoxins [15], with a sensing range of $0.1 \mathrm{pg} / \mathrm{mL}$ to $10 \mathrm{ng} / \mathrm{mL}$, while other MIPs SPR sensors have achieved sensing ranges under $1 \mathrm{nM}[24]$.

\subsubsection{Resonance Light Scattering}

The resonance light scattering sensor is another optical sensor that requires a spectrofluorometer to measure the fluorescent properties of a sample. As shown in Figure 3 , the basic components of these sensors are a light source, an excitation monochromator, the sample, an emission monochromator, and a detector [25]. Normally a xenon lamp, the light source applies a continuous wide band white light. The monochromator is a device 
that allows only a single wavelength with a short bandpass of the light to pass through, which is applied onto the sensing surface. The light is reflected off the sensing surface and the emitted light will have longer wavelength than the emission, so another monochromator is set to filter out all light except for the return wavelength. This change in wavelength is due to the constructive interference caused by Rayleigh scattering. The returned light is then detected, and the intensity of the signal is proportional to the concentration of scattering particles and particle size. The synchronous luminance equation is used to characterize the intensity of the scattered light is given by:

$$
I_{R L S}=k c b E_{e x} \lambda_{e x} E_{e m}\left(\lambda_{e x}+\Delta \lambda\right)
$$

where $\mathrm{k}$ is a constant of the material, $\mathrm{c}$ is the concentration of fluorescent material, $\mathrm{E}_{\mathrm{ex}}$ and $E_{e m}$ are the intensities of the emission spectrums of the excitation and emission, $\lambda_{e x}$ is the wavelength of the excitation and $\Delta \lambda$ is the shift in wavelength between the emission and the excitation. For a constant size of sample, an increase in the concentration of the sample will increase the c factor, increasing the intensity of the resonant lights [26].

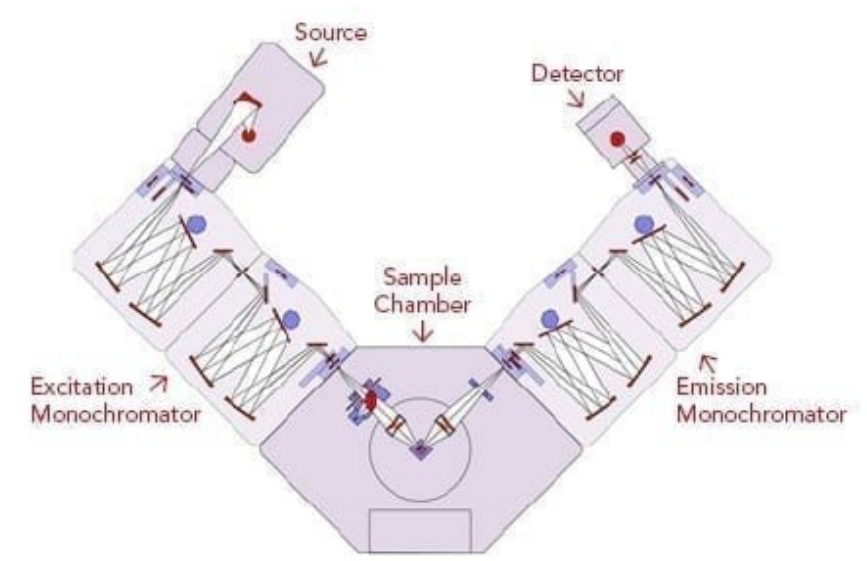

Figure 3 - RLS Sensor Design (reproduced from [100]) 
Works by Zhang et al. have achieved a limit of detection $6.2 \mathrm{pmol} / \mathrm{mL}$ utilizing a magnetic MIPs structure for the detection of the hepatitis A virus [27], while Liu et al. utilized a thermosensitive imprinted polymer to obtain a limit of detection of $1.1 \mathrm{pmol} / \mathrm{mL}$ [16].

\subsubsection{Quartz Crystal Microbalance}

While the optical sensors are better suited for liquid-phase testing, the Quartz Crystal Microbalance (QCM) sensor is a piezoelectric sensor that is designed to work with gaseous samples [28]. The basic structure of a QCM sensor is shown in Figure 4, where a thin piezoelectric quartz crystal is placed between two metal electrodes, and an alternating current is applied between the electrodes. This excitation signal will cause the crystal to vibrate at its resonant frequency, which can be modeled with an equivalent circuit.

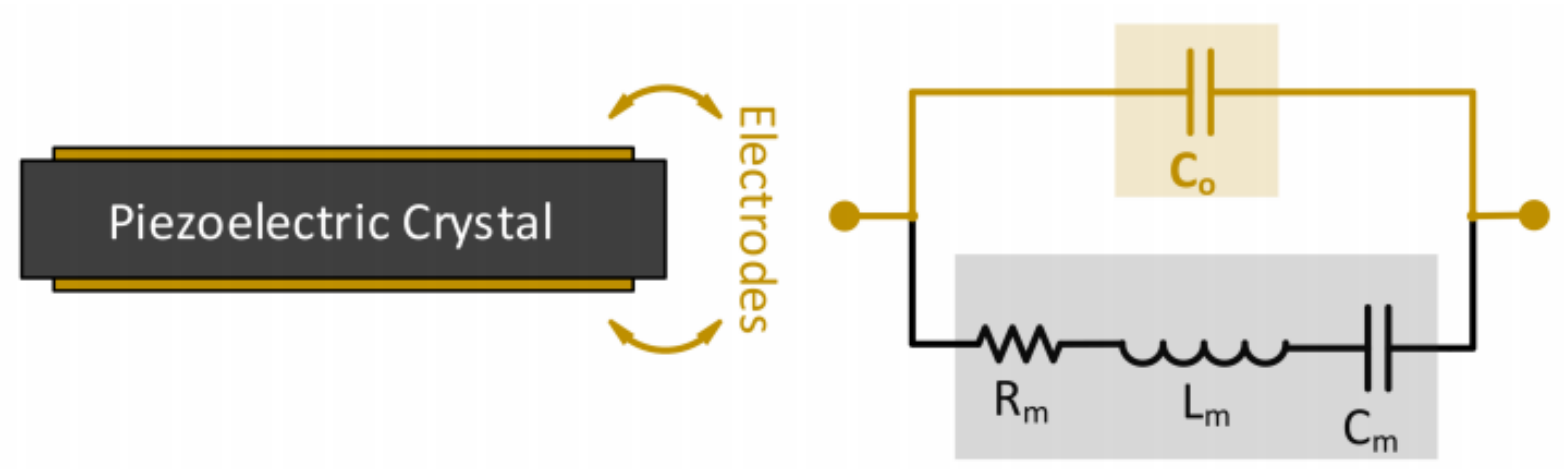

Figure 4 - QCM sensor and equivalent circuit model (reproduced from [29])

When MIPs surface is added to the crystal, the binding of molecules will increase the mass of the crystal, which changes the resonant frequency from equation 1.4, where $\Delta m$ is the change in mass, $C_{c}$ is the mass sensitivity constant of the crystal, and $\Delta f$ is the change in resonant frequency [30]. 


$$
\Delta m=-C_{c} \Delta f
$$

This requires much less components than the optical sensors and is easier to integrate into a complete system as a simple frequency counter can be used to monitor the frequency change. A molecularly imprinted polymer can be deposited over the piezoelectric crystal form the sensing element [31]. Due to the mass added by the bound molecules being extremely small and the mass sensitivity constant normally being relatively small, these sensors normally have a worse limit of detection than other types of sensors, normally in the $\mu \mathrm{M}$ range. As the transducer element can only detect the addition of mass to the crystal, these sensors require very high selectivity in order to function properly [32] [32]. Jha et al. have achieved a limit of detection of $0.3 \mu \mathrm{M}$ in gas samples for the detection of aldehydes [17], while Mustafa et al. have achieved a limit of detection of $46 \mu \mathrm{M}$ for the detection of VOCs [33].

\subsubsection{Surface Wave Acoustic}

Surface acoustic wave (SAW) sensors are another form of piezoelectric sensors, relying on microelectrochemical systems (MEMS). The SAW sensor consists of an input transducer, an output transducer, and a piezoelectric substrate. An electrical signal is applied to the input transducer, which gets converted to a mechanical wave along the surface of the substrate [34]. The mechanical wave is then sensed by the output transducer, measuring the amplitude, frequency, phase, and time-delay between the input and output. For MIPs based sensors, the imprinted polymer is placed over the piezoelectric substrate, 
and as analytes bind the surface characteristics will change, leading to a detectable change in the output wave properties [35].

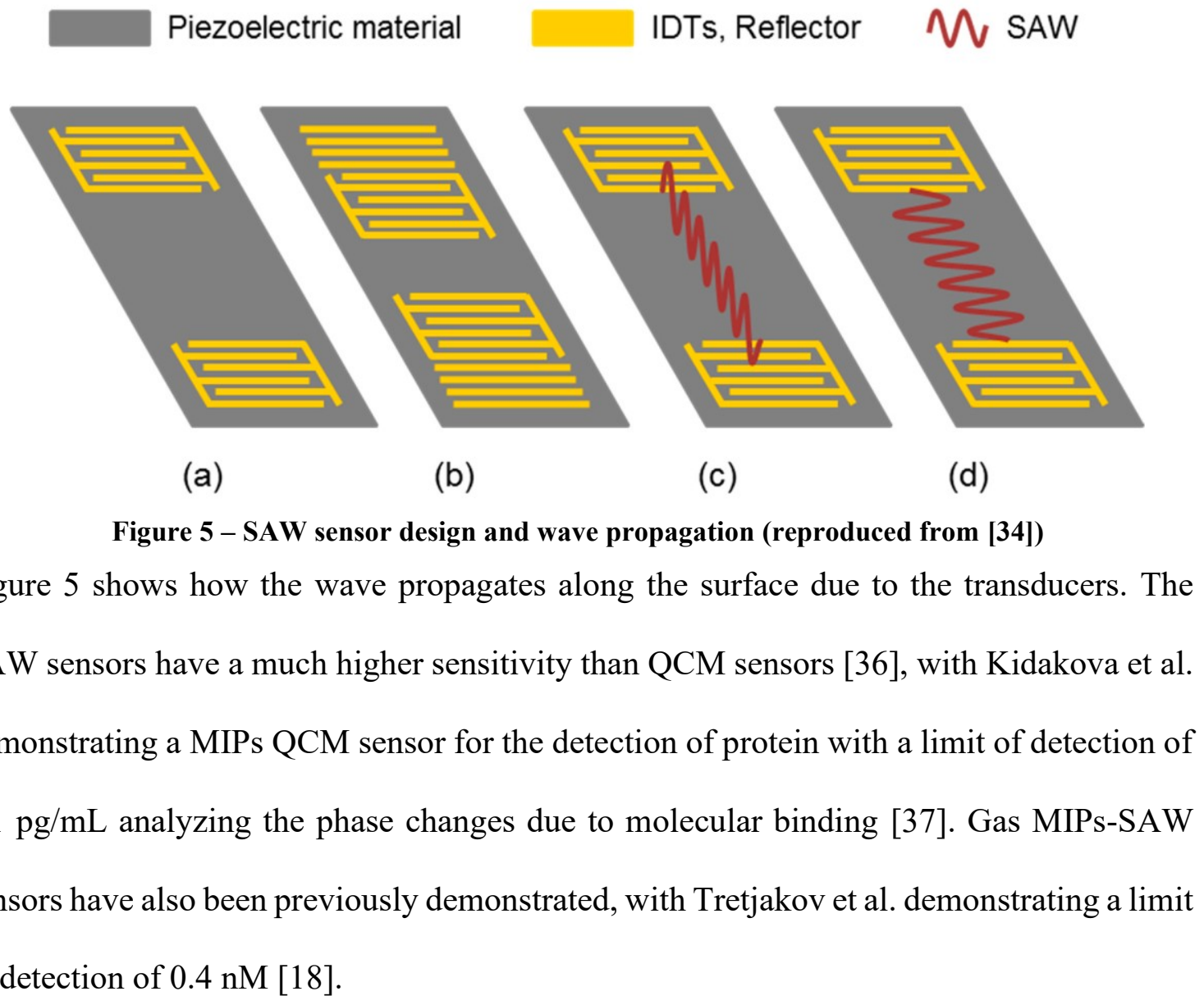

\subsubsection{Field Effect Transistor}

With a chemical field effect transistor, the transistor is constructed with the gate, drain, and source; then a sensing surface is coated onto the gate [38]. The transistor is biased, and the source-drain current is measured. As analytes bind to the sensing layer, the added molecules will affect the net gate voltage and thereby affect the electrical conduction through the transistor leading to a change in the source-drain current. The change in current can then be correlated to the concentration of analytes bound to the 
sensing surface. Once the target has bound to the polymer membrane, the change in effective gate voltage will depend on the conductivity of the polymer. A high conductivity polymer will create a larger effect on the gate voltage, increasing sensor sensitivity.

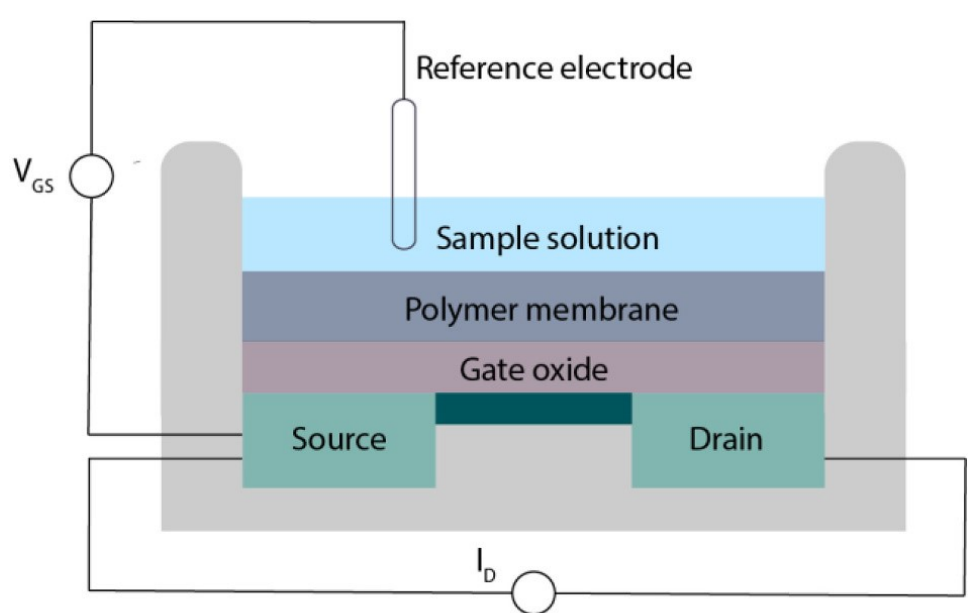

Figure 6 - CHEMFET sensor Design (reproduced from [99])

The sensor design is shown in Figure 6, demonstrating their typical use with solution samples. The polymer membrane can be a MIPs layer and can achieve very high sensitivity and targe a wide range of analytes. These MIPs-Sensors have mostly been used for liquid-phase sensing and are better suited for larger structures such as proteins. Previous works have demonstrated limits of detection down to the $0.1 \mathrm{pM}$ range [19].

\subsubsection{Capacitive}

The capacitive MIPs sensor is an impedance based electrochemical sensor. A high dielectric imprinted polymer is coated over a capacitive structure, either parallel plate or and IDE [39]. When an electrical signal is applied across the electrodes, the electric field will extend out from the fingers and cross through the polymer layer [40]. As shown in Figure 7, as analytes bind the MIPs surface, the effective dielectric seen by the electric 
field will change, creating a detectable change in the capacitance between the electrodes. This change in capacitance can then be correlated to the concentration of analytes bound to the MIPs coating.

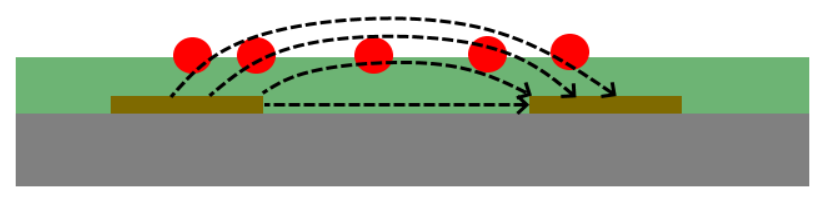

Figure 7 - Capacitive Sensor Design

Capacitive sensing has a wide variety of previous MIPs works, with both gas and liquid phase sensing [20]. The thickness of the polymer layer is essential as the magnitude of the electric field lines will rapidly decay the further it is from the electrodes and if the layer is too thick, there may be no significant electric field at the binding sites. The readout system of this system can be a simple one data point collection to determine its capacitance, or a frequency sweep of the impedance can be done to generate a complete impedance model and extract the capacitance value. These sensors have been shown to have very low limits of detection however tend to have worse sensitivities over other sensor options [28]. Previous works have shown limits of detections under $1 \mathrm{pM}[4]$.

\subsection{Motivation}

Despite the vast number of MIPs based sensors research reported [41], the application of the technology towards exhaled breath condensate sensing has remained limited despite its promising characteristics. Exhaled breath is a non-invasive method for monitoring health [3], especially pulmonary health, the development of a low-cost, long 
shelf-life, and rapid response sensor can have a large impact on health monitoring technology. These devices can provide real time feedback for rapid diagnosis or can also be used to analyze many samples faster than currently used sensors. One important biomarker in exhaled breath condensate is 8 -iso Prostaglandin F2 $\alpha$, which is a marker of oxidative stress [2]. Oxidative stress is caused by an imbalance of oxygen-containing freeradicals and antioxidants in the body, which can be caused by a variety of dietary, lifestyle, or environmental factors. 8-isoprostane has been shown to increase in concentration in the presence of oxidate stress as a product of free-radical catalyzed peroxidation of arachidonic acid [42]. In a healthy adult, it can be found ranges of $\sim 30 \mathrm{pg} / \mathrm{mL}$, increasing up to $>80$ $\mathrm{pg} / \mathrm{mL}$ in severe cases of interstitial lung disease [43]. It can therefore be used for the detection of certain diseases such as asthma, chronic monitoring of obstructive pulmonary disease, and bronchiectasis. It has been used for early-stage detection, as oxidate stress will start to occur before a patient might notice symptoms. Current 8 -isoprostane sensing techniques require complicated lab-based detection methods such as infrared spectroscopy [44].

This work demonstrated that the generic MIPs method can work with small molecules and could therefore be applied to a wide range of biomarkers beyond 8isoprostane. A complete analysis of key exhaled breath biomarkers could be accomplished using the technique that was researched throughout the project. The application of MIPs technology towards exhaled breath monitoring has been shown to be viable, and a twopoint capacitive sensing method has been demonstrated to improve the sensor response. 


\subsection{Thesis Organization}

Chapter 2 provides an in-depth literature review on MIPs based sensors with a focus on gas sensing. Several prominent methods of detection are presented with examples of existing sensors that utilize these technologies. The advantages of each sensor type are presented, and the reasoning behind why capacitive sensing was selected is explained. In chapter 3, the theory of operation of the presented sensors is explain in-depth. The reasoning behind the material selection is presented, and the IDE geometry is analyzed and explained. Chapter 4 focuses on the materials and methods, providing details on manufacturing of the sensor stamp and the imprinting method, as well as the testing setup and methodology for aqueous and aerosolized solutions. In this chapter, the equivalent circuit model is introduced along with the governing equations. The focus of chapter 5 is on experimental results, starting with an analysis of the surfaces of the sensor stamps and the imprinted surface. The model extraction results are compared to the measured data to quantify the accuracy of the model, which is then used to quantify the sensitivity and selectivity of sensor towards 8-isoprostane. This analysis utilizes molecules of comparable molecular mass that are also found in exhaled breath condensate, as well as the time domain measurements to determine sensor response time. The conclusion and future work sections discuss the outcomes of the reported works and indicates further project objectives that are currently in progress with the objective of improving the integration of the sensor towards a point-of-care system. A more detailed explanation of the board design is provided in the appendix, however as the board contains over 120 components this section will only highlight the most important design considerations. 


\section{Chapter 2: Literature Review}

\subsection{Resistive vs Capacitive Sensing}

For thin-film impedance-based sensing, the two most common sensors are the resistance and capacitance sensors. These sensors have demonstrated low-cost sensing with both liquid and gas samples, and recent advancements in the technology has shown comparable limits of detection. Both sensors involve coating a sensing material over an interdigitated electrode (IDE) structure, which is designed differently depending on type of sensor.

The resistive sensor utilizes a conductive sensing layer, such as a modified tin or titanium oxide film, usually patterned directly over an IDE structure. When exposed to a target analyte that binds to the sensing layer, the conductivity will change, and the resistance between the electrode's changes. This resistance is the transconductance parameter of the sensor. The resistive sensors are normally classified into two groups depending on the material used for the sensing layer, which is normally either a MetalOxide Semiconductor (MOX) or a conductive polymer. While the MOX resistive sensor traditionally has a very high sensitivity and has high power consumption and is limited in sensor coatings and the possible analytes it can detect. The conductive polymer sensor is cheaper to develop and can be used for a wider range of targets, especially VOCs [45]. The advantage of the resistive sensor over the capacitance sensor is a simpler design. The IDE design is less critical for the resistive sensor as the conductivity can be measured in DC or a single frequency. A DC resistance measurement is more susceptible to noise, and the sensitivity of these sensors is normally restricted by the noise. This sensitivity can be 
improved using an ac measurement at the cost of introducing complexity to the sensor readout circuitry.

One critical aspect of the IDE design for these sensors is the finger spacing, since increasing the space between fingers lowers the current density if the voltage is kept constant at DC. Reducing the space between the fingers can be used to maintain a desired current density while operating at a lower voltage. The readout circuitry for such as sensor is also quite simplistic, with a simple voltage divider or transimpedance amplifier feeding a DAC enough to measure the resistance. One disadvantage of the resistor sensors is its dependence on temperature, MOX chemiresistors normally require a high operating temperature and therefore require a heater to function, although the conductive polymer chemiresistors do not have this disadvantage. However, the conductive polymer is more susceptible to temperature variations, so a heater is still recommended to maintain on constant temperature.

One such chemiresistive sensor was demonstrated in the co-authored paper outlined in the authorship section of this report shown in Figure 8, along with a labeled IDE structure. The sensing layer is a functionalized graphene oxide, moving away from the traditional metal-oxide sensor. Due to its porosity, graphene has a high surface to volume ratio which is ideal for gas sensing [46]. However, the conductivity is too high to use for a resistive element. As it oxidizes, the conductivity will decrease, and the functionalization process will decrease it further, allowing it to act as a resistor. To illustrate, graphene oxide (GO) can be fluorinated to create fluorinates graphene oxide (FGO) creating C-F bonds which will interact with certain analytes, specifically ammonia [47]. 


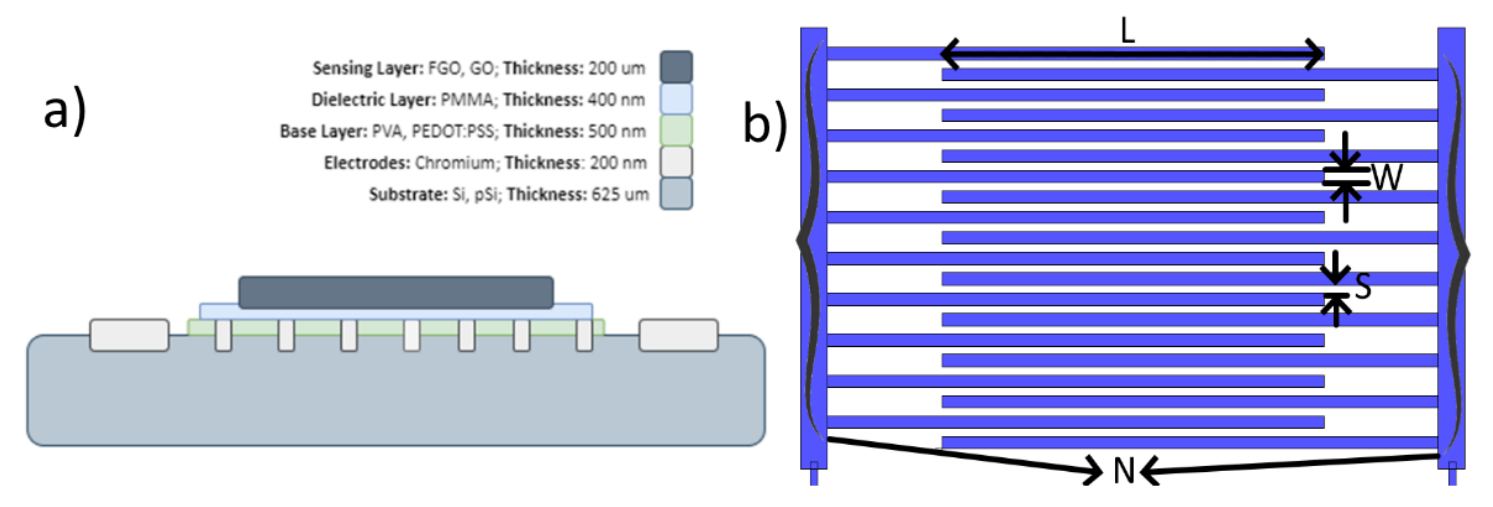

Figure 8 - (a) Example of a Multi-Layer Resistive sensor for $\mathrm{NH}_{3}$ Sensing (reproduced from [47]) (b)

Basic IDE structure with Important Dimensions

The capacitive sensor also uses a sensing layer coated over an interdigitated electrode structure; however, the properties of the sensing layer must be high dielectric to have a high capacitance [40]. The sensing mechanism is the change in the effective dielectric seen between the electrodes, as previously shown in Figure 7. The sensing material is normally composed of high surface to volume materials, such as nanofibers, nanoparticles, or porous polymers [48]. The interaction between samples and the sensing layer can be faradic or non-faradic, depending if reduction-oxidation reactions occur on the sensor surface. A key design element for capacitive sensors is to reduce the sensing layer as much as possible as the electric field from the relatively small capacitance between the electrode fingers cannot extend very far from the electrode surface.

Due to the minimal effect of temperature on both the MIPs surface and the capacitance, no heater is required for the operation of the sensors. However, the readout design is much more complicated than the resistive sensor, requiring an impedimetric measurement over a frequency range and matching of the measurement to a model to 
extract the capacitance value. The IDE parameters must be selected to set the required frequency range, and optimization of the parameters can increase sensitivity, increase nominal capacitance, and reduce the frequency range for faster analysis. With a properly optimized IDE the impedimetric measurement can be sped up by only measuring known key frequency points. The IDE design for capacitive sensors involves optimizing the ratio between the electrode-sensing layer area and the distance between the fingers to ensure the electric field going through the sample is as large as possible.

Due to generic MIPs material used in the reported works, there is little to no change in the polymer material properties as the templates become lodged in the imprinted cavities. For capacitive sensing, no chemical interaction between the template and polymer is required, as long as the templates are inside the nanocavities the change in effective dielectric can be measured. Furthermore, the selected PVA-SbQ has low conductivity and is poorly suited for resistive sensing. Finally, previously reported MIPs gas sensors have mostly used capacitive sensing to achieve very low limits of detection, so it is believed that the generic material approach is feasible. For these reasons, the capacitance sensor was selected for this research.

While the main transconductance parameter for the presented sensors is the capacitance, the complete impedimetric model (containing resistors and capacitors) is used to improve the sensor readout as well as the selectivity. As the MIPs material shows bonding between the target and the polymer, this sensor is susceptible to cross-selectivity, as molecules similar to the target may become lodged in the cavities. The complete model, along with a reference non-imprinted sensor, allows for the filtering of non-specific binding 
as it will also affect the other model parameters, while the template molecule has little effect on said parameters.

\subsection{Materials for Molecularly Imprinted Polymers}

Although there a wide variety of ways in which molecularly imprinted polymers are prepared, some of which are covered in section 2.3 , they normally consisted of functional monomers, cross-linkers, and a template [49]. When combined and polymerization is induced, the cross-linkers will link the functional monomer groups into a polymer that are bound to the templates. Once the templates are removed, nano-cavities are left in the polymer surface which have high affinities towards the templates. Determining what materials to use is often the most complicated part of developing MIPs sensors, as the materials and ratios of template/monomer/cross-linker must be carefully selected to achieve a high-quality imprinted polymer. Due to the complicated reactions that occur and the large among of variables, a rational design approach can be a long and complicated approach, with some incorporating computer simulation to rapidly analyze a multitude of monomers. These approaches can utilize a variety of analysis methods, from molecular analysis to quantum mechanics.

The main criteria for monomer selection are that functional groups of the monomers and the template must match or be complementary in order to be able to form complexes with each other. The more interactions that occur between the monomers and templates, the better the MIPs will be. The monomer size should not be larger than the template, as this reduces the interactions between the two. The cross-linker ratio should decrease with increasing template size, with small template molecules the reaction will require a large 
ratio of cross-linkers. In general, an experimental process is required to develop a highquality MIPs through trial and error.

Recent work, as well as the presented work, seeks to analyze a more generic approach to the development of MIPs based sensors. Poller et al. [8] have demonstrated the application of a "ready-to-use" material that has not been specifical tailored to the analyte. While the success of this method will vary depending on the coating material and analyte, it was shown that this proposed method can even surpass the sensitivity of conventional synthesized polymers. This method was demonstrated in the detection of bacteria, however as MIPs technology has been shown to be easily scalable for different targets, the same method was used for small molecule detection. One major advantage to this method is that the reproducibility is much better over the custom synthesis methods. With such a wide range of variables in the synthesis of a custom polymer, it is a long and difficult process to attempt to ensure good reproducibility. With the polymer already premade, partially polymerized, and combined with cross-linkers, the MIPs production time and difficulty of synthesis can be reduced.

\subsection{Molecularly Imprinted Polymers Fabrication Methods}

The fabrication techniques of MIPs are just as varied as the types of sensors, which can be divided into three main categories: direct synthesis, phase inversion, and soft lithography, however there are many more possible methods [50].

\subsubsection{Synthesis}

The most general production method, synthesis involves allowing the monomers and templates to interaction in solution, which can have either covalent or non-covalent 
interactions [51]. The monomer is selected to have functional groups that will interact with the template, such as hydrogen-bonding functionality that can form a covalent bond with a template. Common functional monomers are those that have been found to have interacted with a variety of templates, such as acrylamide, methyl methacrylate, methacrylic acid, aniline, and pyrrole [52]. After the monomers and templates are mixed, a cross-linker and a polymerization agent are added. The cross-linker, such as ethylene glycol, is used to connect the monomers in the polymer chains, and the imitator, such as azobis(isobutyronitrile), forms intermediate bonds between monomers to allow them to then link into larger polymer chains. Polymerization is then induced with normally either heat or UV light, and this process is allowed to continue until it forms into a powder. This process is normally very time consuming and may require up to 24 hours to allow for the polymerization to complete. The templates are then removed using a variety of techniques, such as washing the polymer in a solution that will dissolve the template but to which the polymer is insoluble. This process is outline in Figure 9, and can be used to form films instead of powders if the reaction is allowed to occur on a substrate, however the long production time can be impractical and difficult to generate a controlled layer that is useful for a sensor. The advantage of the powder is that it has a long shelf life and can be mixed into a variety of other materials. 

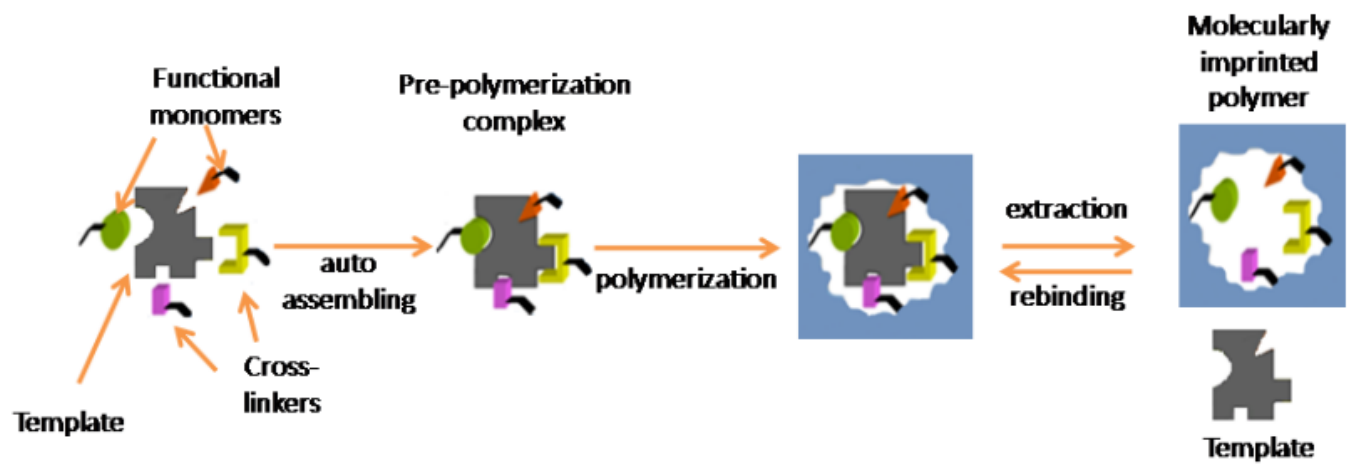

Figure 9 - MIPs synthesis technique (reproduced from [53])

Variations of this method exist, such as a solid-state synthesis which bonds the templates to a solid support structure, such as glass beads, before mixing with the monomers. After polymerization, the solid supports can be easily removed to rapidly separate the templates from the imprinted polymer. Another method is to use magnetic supports for the templates so that a magnet may be used to remove the templates [27].

\subsubsection{Phase Inversion}

A faster approach than synthesis, this method starts with polymer that has already been linked, as opposed to starting from the functional monomers. The polymer and template are mixed together, and both are dissolved together with the addition of a solvent. The polymer and template combine in this mixture, and a weak solvent is added to cause the imprinted polymer that is bound to the template to separate, leaving behind a solid polymer. 


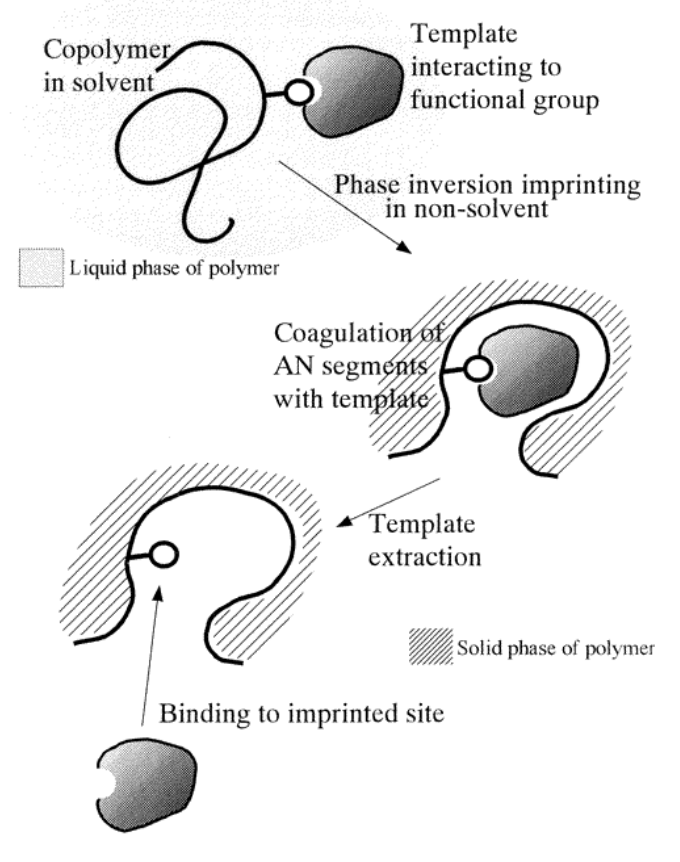

Figure 10 - MIPs Phase Inversion technique (reproduced from [54])

The imprinted polymer can also be separated from the solvent through other methods such as evaporation. Since there is no cross-linker, relative to synthesis this method will generate a less dense, less homogenous, and less sensitive binding sites. This imprint method is illustrated in Figure 10, showing the change in phase.

\subsubsection{Soft Lithography}

Ideal for thin-film production, soft lithography allows for the imprinting process to be done directly on the sensor surface. The template molecules are bound to a stamp, which is normally a transparent support such as PDMS. As shown in Figure 11, a partially polymerized film, such as PVA-SbQ, is then deposited onto the sensor surface, normally through spin-coating to control the thickness of the layer. The stamp is then pressed into the partially polymerized film, and cross-linking is imitated. Once the layer has completely polymerized, the stamp can then be removed, removing the template with it [55]. 

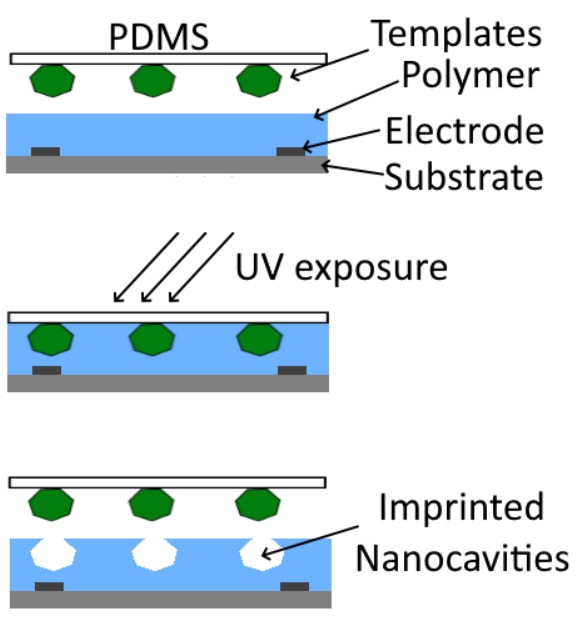

Figure 11 - MIPs Soft Lithography Technique

A major advantage of this method is the stamp, as it can be reused multiple times. This leads to lower fabrication time and will ensure better uniformity in performance between batches of sensors. Due the use a partially polymerized starting material, the imprinted polymer normally has higher sensitivity than those created from phase inversion but lower than those created from synthesis. 


\section{Chapter 3: Theory}

\section{1 "Generic" Molecularly Imprinted Polymers}

Molecularly imprinted polymers have been widely used for sensing applications due to having a large sensing area, making it ideal for low concentration detection. This large sensing area allows the material to be used for both aqueous and gas sensing containing biological samples depending on the type of sensor [41]. Molecularly imprinted polymers have often been called plastic or synthetic antibodies, whose structure is a welldefined polymer that has a very specific spatial conformation and is mostly stabilized by weak interactions [56]. The molecular recognition capability of antibodies comes from the three-dimensional structure with specifically located functional groups. A molecule will only bind to the antibody if it has compatible functional groups at the same location as the antibody and must fit within the 3D structure. All the functional groups within antibody side chains can be mimicked with monomers and using synthetic polymers additional functionality can be introduced by using functional groups not found in antibodies. The difficulty in using synthetic polymers instead of antibodies comes from radical polymerization being a random process, making reproductivity almost impossible. This difficulty is overcome by introducing the template molecule during polymerization, as the spatial configuration of the polymer is forced to conform around the template. This process is where the term "molecular imprinting" was derived. Unlike antibodies which are mostly rely of weak interactions to maintain its shape, the synthetic polymer requires a strong cross-linking to maintain its structural properties. 
Previous works have studied the comparative performance of MIPs based sensors and antibody-based sensors. When used for targeting small molecules in the enzyme-linked immunosorbent assay (ELISA) format, the MIPs sensor has achieved similar or better performance than the antibody sensor [57]. This was proven using the synthesis imprinting method, which normally has the best performance, and with carefully selected materials. The MIPs sensor was proven to detect concentrations down to the picomolar range and has better stability and shelf life. Between the use soft lithography instead of synthesis and the lack of a specifically curated polymer for the template, it is expected that sensor outlined in this research will have reduced performance as a trade off for the simplicity in design and fabrication. As outlined in the results section, this reduced performance in mostly in the selectivity of the sensor, as the selected materials are still ideal for ultra low concertation detection.

The binding process during imprinting can be classified into two groups: covalent and non-covalent [58]. The most common imprinting method is non-covalent, meaning that the interactions between the template and the polymer consist mostly of hydrogen bonding, electrostatic effects, van der Waals forces, and the hydrophobic effect [56]. The imprinting process using covalent bonds is more complex as the bonds must be reversed in order to remove the template after imprinting, but the advantage is normally an improvement in the selectivity of the sensor. With the generic polymer used in this research, noncovalent bonding is used, mostly relying on the hydrogen bonding, which has the advantages that bound molecules can be easily removed from the polymer after a test. To expand this research to molecules with different functional groups, a different polymer material will be required and tested to ensure it could work together. 


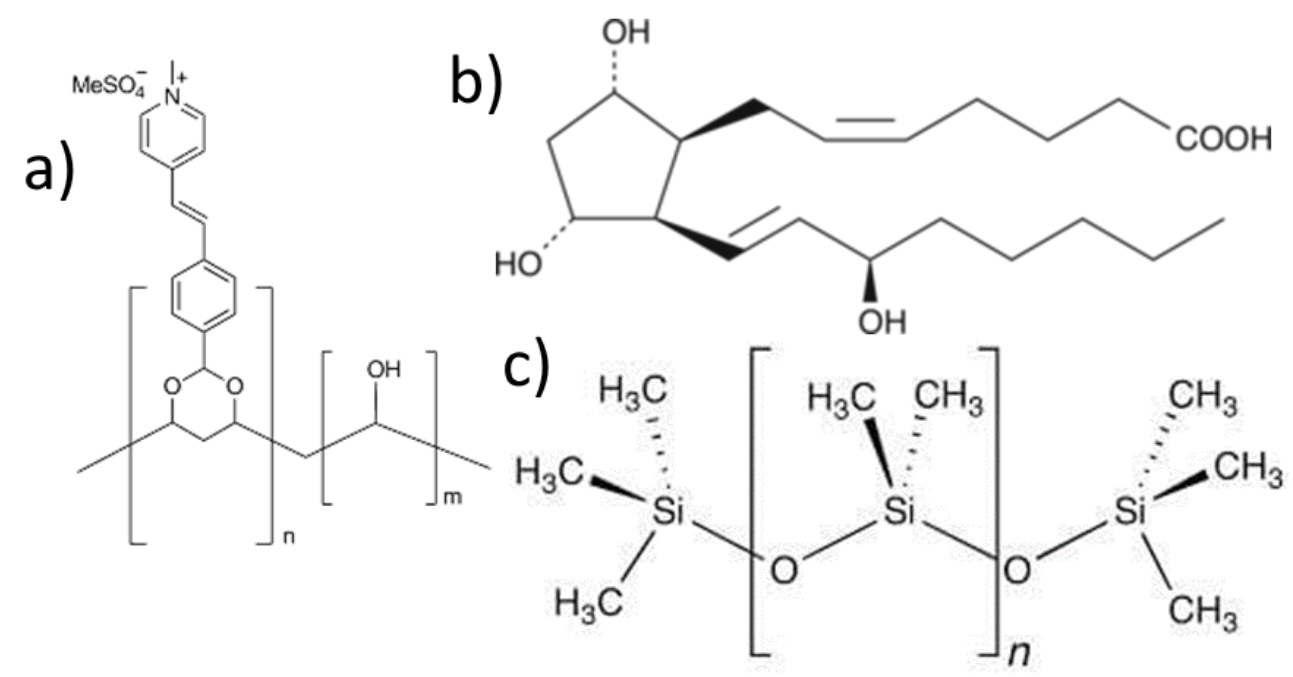

Figure 12 - Molecular structure of: (a) PVA-SbQ (reproduced from [59]) (b) 8-isoprostane (reproduced from [60]) (c) PDMS (reproduced from [61])

The molecular structures of the materials used is shown in Figure 12. Polyvinyl Alcohol (PVA) is a water-soluble synthetic polymer that has been used in a wide variety of biomedical applications due to being biocompatible and non-toxic. It has been used for cartilage replacement, contact lenses, temperature, and humidity sensors [62], and thinfilm sensors including molecularly imprinted sensors. Once polymerized, it forms a hydrogel that is highly absorbent [63], however it does not dissolve in water and will maintain its structure [64]. For sensors applications it can used to form a functionalized hydrogel, either by creating a mixture with the functional material or through molecular imprinting. PVA as been shown to be highly effective at the entrapment of biologically relevant samples from molecules to enzymes and microbial cells [65]. PVA is often photo crosslinked, developing a high durability, flexible hydrogel that can adhere to silicon wafers [66].

Poly (vinyl alcohol), N-methyl-4(4'-formylstyryl) pyridinium methosulfate acetal (PVA-SbQ) is a high dielectric photo-cross linkable polymer derived from PVA. The 
styrylpyridinium group acts as the cross-linker, which can be initiated via ultraviolet light. Only about $1 \mathrm{~mol} \%$ of SbQ has been shown to enough to link the PVA chains together, however as the PVA-SbQ solution used is a dilute solution, a $4.1 \mathrm{~mol} \% \mathrm{SbQ}$ is required to achieve cross-linking [67]. This low SbQ content means that the chemical and physical properties of the PVA-SbQ and unmodified PVA are essentially identical. Despite being around for more than 30 years, the exact photosensitive characterised of the PVA-SbQ are not well understood, especially the ability to cross-link with such a low SbQ content. A recent study has demonstrated that the structure assembly is though h-type aggregate, meaning that molecules will stack mostly face-to-face. The absorption spectra of PVA$\mathrm{SbQ}$ before and after polymerization shows a blue-shifted band at $328 \mathrm{~nm}$ which is understood to be a precursor of photodimerization in solutions and films. The PVA-SbQ is a partially polymerized material, so it does not require an initiator, making it ideal for the soft lithography MIPs production method. The formation of the PVA-SbQ structure is due to the formation of cyclobutane rings formed by the styryl conjugation due to the UV crosslinking. This well-formed structure after the crosslinking is what changes the solubility of the PVA-SbQ.

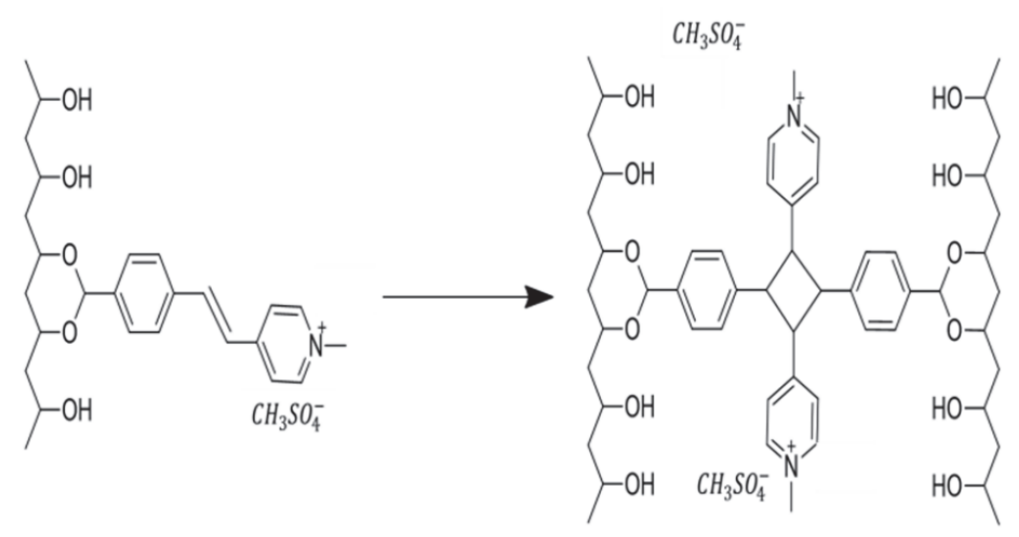

Figure 13 - PVA-SbQ crosslinking structural change (reproduced from [68]) 
Both PVA-SbQ and 8-isoprostane contain multiple -OH functional groups, which will form weak hydrogen bonds during the polymerization process. Due to the generic imprinting method, the other non-covalent interactions are not considered for this sensor as they will change from template to template while the presence of hydrogen bonds between the polymer and potential templates is easily identifiable. The PVA-SbQ has a quick polymerization time at 30 minutes under a $325 \mathrm{~nm}$ UV lamp. Due the presence of the hydrogen bonding, the highly desirable properties of PVA, and quick polymerization time, and its usability for surface imprint, the PVA-SbQ was deemed a suitable polymer.

The 8-isoprostane template will bind the PVA-SbQ through the hydrogen during the cross-linking process, however the force is weak enough that the 8 -isoprostane will be removed during the stamp lift-off process. Due to the weak force of the hydrogen bond and the ultra-low concentrations of the template during a test, it is expected that there will be little to no noticeable bonding when examined with FTIR, and that most of the interaction between the imprinted polymer and the template being due to shape of the imprints.

Polydimethylsiloxane (PDMS) was selected as the stamp material as it is commonly used for soft lithography, most often for microfluidic channels [69]. PDMS polymerizes into a hydrophobic elastomer, meaning that it won't absorb any water from the PVA-SbQ during the imprinting process [70]. The PDMS can be easily patterned by curing it over a template. The elastic properties help it retain its physical properties during repeated use of the stamp. PDMS cures transparent and can therefore be used for UV induced polymerization. This material also retains its properties over a wide range of temperatures and is low cost and easy to fabricate. 


\subsection{Sensor Model}

Once the sensor polymer has been developed, the next step is determining how to measure the sensor response. Impedance spectroscopy is used to measure the impedance between the electrodes, and the data can be used to determine the concentration of the analyte on the sensor [71]. In order to analyze how the MIPs film properties and the IDE structure effects the impedimetric response, an equivalent circuit model (ECM) is used [72]. The ECM is composed of only passive components as there is no power generated within the system. In the ECM, every component corresponds to a physical element of the sensor, and can be constructed using resistors, capacitors, inductors, and constant phase elements (CPE) [73]. The resistors, capacitors, and inductors are ideal components, which will not be able to model non-idealities (such as the double layer capacitance) in the sensor, leading to the necessity of the constant phase element. The CPE has an impedance shown in equation 3.1, where the $\alpha$ represents the phase change due the element. If $\alpha=1$, this is equivalent to an ideal capacitor, if $\alpha=0$, the CPE acts as a purely resistive element, and if $\alpha=-1$ the CPE is an ideal inductor. This element can then be separated into its real and imagery components [74].

$$
\begin{gathered}
Z_{C P E}=\frac{1}{C_{\alpha}(j \omega)^{\alpha}} \\
\operatorname{Re}\left(Z_{C P E}\right)=\omega^{-\alpha} C_{\alpha}^{-1} \cos \left(\frac{\alpha \pi}{2}\right) \\
\operatorname{Imag}\left(Z_{C P E}\right)=-\omega^{-\alpha} C_{\alpha}^{-1} \sin \left(\frac{\alpha \pi}{2}\right)
\end{gathered}
$$

The equivalent circuit model can be as simple as single component, or can contain over a dozen components, depending on the complexity of the sensor design and the 
interactions occurring on the surface. With electrochemical impedance spectroscopy, the reactions that occur on the electrode are classified as either faradic or non-faradic [75], which normally lead to different models. While a faradic and non-faradic sensor can have the same model, the physical meaning of the elements will differ. In addition to changes in the sensor resistor and capacitance while under test, there will be additional impedance changes depending on the type of sensor.

In faradic sensors, the current flows through the sensor because of reductionoxidation (redox) along the electrode surface. The reduction-oxidation reactions lead to charge-transfer along the surface, which is frequently used as the sensing element for faradic sensors. The models utilized for these sensors are normally composed of elements that represent the physical characteristics of the sensor and components that model the effects of the charge transfer. Depending on the sensor design, the resistance introduced by the charge transfer is used frequently used as the main sensing element. The non-faradic sensor has no reduction-oxidation on the surface, and the impedance change is due to the formation of a double layer along the surface and the collection of ions on the electrode interface. Depending on the sensor design, the capacitance introduced by the double layer effect may be commonly used as the main sensing element. This double layer effect is a capacitance caused by formation of layers of charge at the electrode-coating interface.

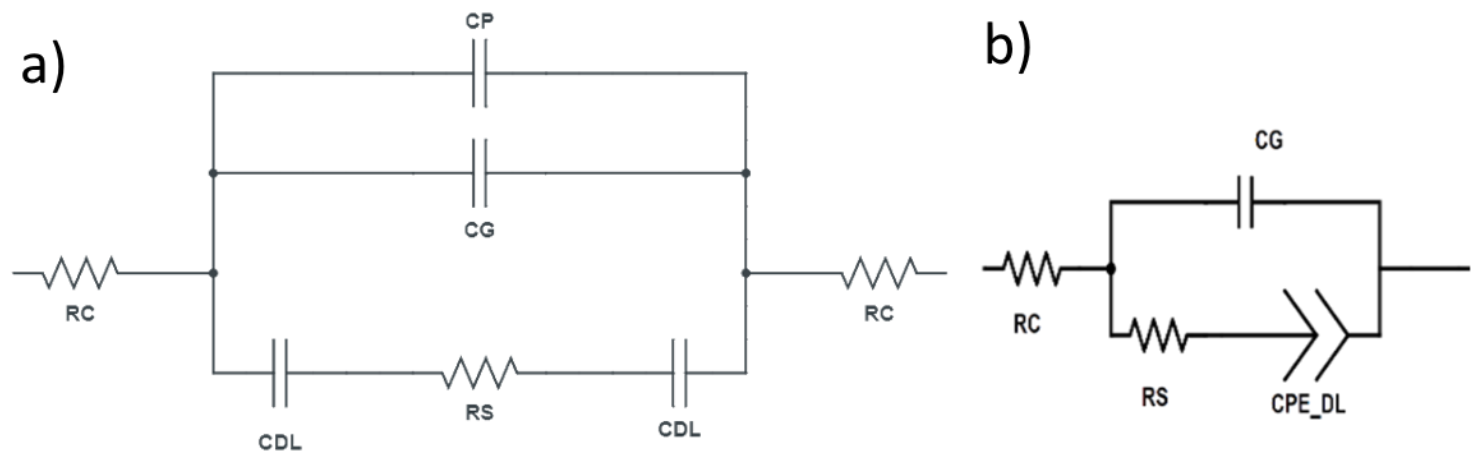

Figure 14 - (a) Full Equivalent Circuit model (b) Simplified Equivalent 
The sensor in this report is non-faradic, as no redox reactions will occur on the surface and no elements for the charge transfer [76]. There is also no diffusion occurring for this sensor, further simplifying the model. The sensing mechanism of the presented sensor is based on the change in equivalent dielectric between the electrodes. The double layer effect leads to an additional capacitance seen in the model, which is used in the detection of non-target analytes on the surface as well as the detection of sensor saturation. The initial complete model is shown in Figure 14, from which the 4-element model is derived. For each electrode there is a contact resistance $\left(R_{C}\right)$, between the electrodes there is the capacitance from the geometry of the IDEs $\left(\mathrm{C}_{\mathrm{G}}\right)$, the double layer capacitance from each electrode to the coating $\left(\mathrm{C}_{\mathrm{DL}}\right)$, and the resistance introduced by the coating between the electrodes $\left(R_{S}\right)$. All parasitic capacitances are grouped in the parasitic capacitor $\left(C_{P}\right)$. These properties were confirmed after performing physical and chemical characterization of the sensor.

To simplify the model, certain elements can be combined. The parasitic capacitance is assumed to be considerably smaller than the geometry capacitance, so the parasitic capacitance is combined into the geometry capacitance. Instead of modeling the double layer capacitance for each electrode, the two are combined into a single double layer capacitance with a series resistor for the coating resistance. The contact resistances are also combined into a single resistance. As the double layer capacitance is an imperfect capacitor, the component is then replaced with a constant phase element. As shown in the results section, the $\alpha$ was normally above 0.9 , indicating that the double layer capacitance was close to ideal. Although not a capacitor, due to the CPE representing the double layer being reasonably close to ideal and in order to keep terms consistent, the double layer 
element will continue to be referred to as the double layer capacitance. The impedance from the 4-element model is shown in equation 3.4. For simplicity, the real and imaginary components in equations 3.6 and 3.7 are derived assuming $\alpha=1$ for a frequency range of 10 $\mathrm{Hz}$ to $100 \mathrm{kHz}$.

$$
\begin{gathered}
Z=R_{C}+\frac{1+(j \omega)^{\alpha} C_{D L} R_{S}}{(j \omega)^{\alpha} C_{D L}+j \omega C_{G}+(j \omega)^{\alpha+1} R_{S} C_{G} C_{D L}} \\
Z=R_{C}+\frac{j \omega C_{D L} R_{S}+1}{j \omega\left(C_{G}+C_{D L}\right)-\omega^{2} C_{G} C_{D L}} \quad \alpha=1 \\
Z=\operatorname{Re}(Z)+\operatorname{Imag}(Z) \\
\operatorname{Re}(Z)=R_{C}+\frac{R_{S} C_{D L}^{2}}{\left(C_{G}+C_{D L}\right)^{2}+\left(\omega R_{S} C_{G} C_{D L}\right)^{2}} \\
\operatorname{Imag}(Z)=-j \frac{C_{G}+C_{D L}+C_{G}\left(\omega R_{S} C_{D L}\right)^{2}}{\omega\left[\left(C_{G}+C_{D L}\right)^{2}+\left(\omega R_{S} C_{G} C_{D L}\right)^{2}\right]}
\end{gathered}
$$

The derivation of these equations is found in the appendix. The 4-element model consists of the two current paths, one consisting of the geometry capacitance and the other consisting of the double layer capacitance and the coating resistance [71]. From the impedance equation, the shape of the impedance magnitude and phase plots are shown in Figure 15. Due to the small area between the IDE fingers and the large area for the double layer capacitance, the geometry capacitance is assumed to be smaller than the double layer capacitance by at least an order of magnitude. At low frequency, the larger double-layer capacitance will have a smaller impedance magnitude than the geometry capacitance but will have a larger impedance magnitude than the coating resistance. The current will then mostly flow through the double layer capacitance - coating resistance path, and since the double layer impedance is larger it will dominate the impedance. As the frequency 
increases, the impedance from the double layer capacitance will decrease until its magnitude is smaller than the coating resistance; this frequency is referred to as $f_{\text {LOW }}$ (divides regions 3 and 2 in Figure 15). At this point the current will still flow through the double layer capacitance - coating resistance path, but since the coating resistance is larger it will dominate the impedance. As the frequency increase further, the impedance magnitude of the geometry capacitance will have decreased until it is smaller than the coating resistance, and the current will mostly flow through the geometry capacitance path; the frequency at this point is referred to as $f_{\mathrm{HIGH}}$ (divides regions 2 and 1 in Figure 15). To be able to extract all the model parameters from the impedance data, all three regions must be properly defined. If the corner frequencies are too close together or too close the frequency sweep limits, there will not be enough data points in that section to cleanly extract the model. Examples of poorly defined regions are shown in Figure 23. In order to ensure that the corner frequencies are properly set, the IDE dimensions and coating properties are analyzed to set $f_{\text {LOw }}$ around $1 \mathrm{kHz}$ and $\mathrm{f}_{\mathrm{HIGH}}$ to around $15 \mathrm{kHz}$. With a logarithmic sweep up to $100 \mathrm{kHz}$, each section will be well defined.
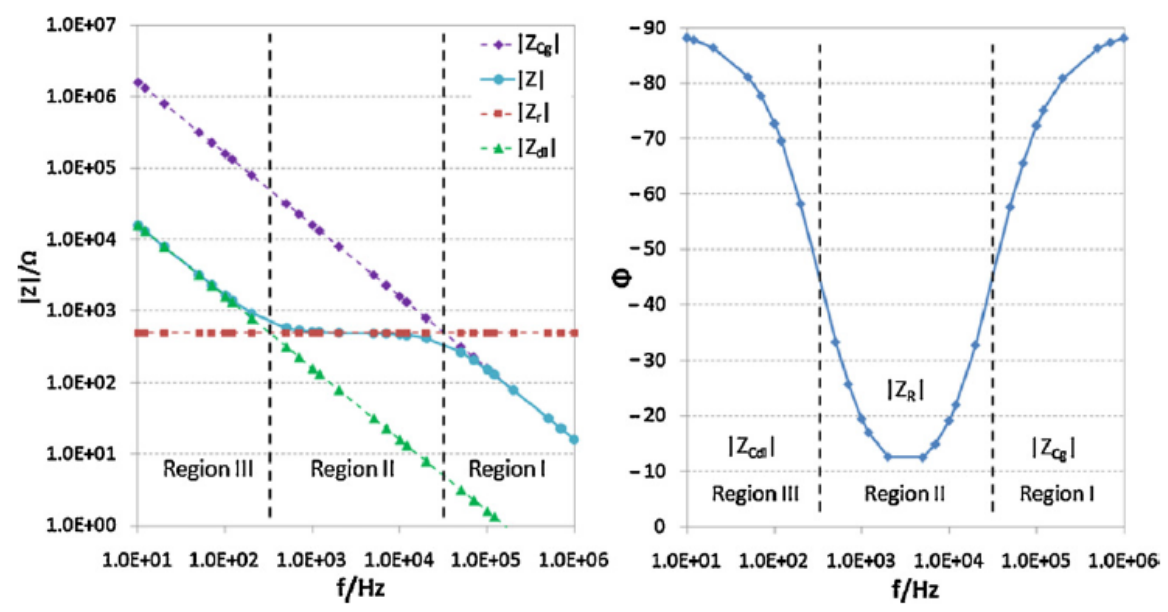

Figure 15 - Impedance Magnitude and Phase from model (reproduced from [71]) 


\subsection{Optimization}

Due to the passivation of the substrate with silicon dioxide which acts an insulator, it is assumed that the effective conductivity is solely from the coating properties. Silicon dioxide has an electrical conductivity of $10^{-12} \mathrm{~S} / \mathrm{cm}$, while the expected conductivity of the hydrogel is expected to be in order of $10^{-6} \mathrm{~S} / \mathrm{cm}[77]$. The relative dielectric constant of the silicon dioxide of $\sim 3.8$ is also expected to be at least an order of magnitude lower than the relative dielectric of the hydrogel, which is expected to be around 80 [78], and therefore it is also assumed that the effective dielectric seen between the electrodes is purely from the PVA-SbQ polymer. The thickness of the silicon dioxide is also assumed to be large enough that no electric field lines will reach the substrate, so the effects of the substrate can also safely be ignored.

Using equations 3.8 to 3.11 , the coating resistance can be calculated knowing the solution conductivity and the cell constant. The cell constant $\left(\mathrm{K}_{\mathrm{CELL}}\right)$ is a constant that depends entirely on the sensor geometry and is determined by the ratio of the distance between electrodes and area of the electrodes [79]. Between the two fingers, the geometry capacitance depends on the cell constant and the dielectric of the coating material. At the surface of the electrode and the coating, the double layer will depend on the materials at the surface and the area of the electrodes. A constant labeled $\mathrm{C}_{\mathrm{DL} \text {,Surface }}$ is a characteristic of the PVA/Sample mix and the electrode interface. The $\mathrm{K}_{\mathrm{CELL}}$ constant has been defined by Olthuis et al. [80], and the IDE parameters are given by S, W, L, and N, being the finger spacing, width, length, and number of fingers. The cell constant is the effective ratio of the gap between the electrodes and the area of the electrodes. 


$$
\begin{gathered}
R_{S}=\frac{K_{C E L L}}{\sigma_{S}} \\
K_{C E L L}=\frac{2}{L(N-1)} \frac{K(k)}{K\left(\sqrt{1-k^{2}}\right)} \\
K(k)=\int_{0}^{1} \frac{1}{\left(1-t^{2}\right)\left(1-k^{2} t^{2}\right)} d t \\
k=\cos \left(\frac{\pi}{2} \frac{W}{S+W}\right)
\end{gathered}
$$

For the interdigitated electrodes, the capacitance from the geometry and the double layer capacitance are given in equations 3.12 and 3.13 [79]. The geometry capacitance depends on the dielectric constant of the coating and the cell constant. The double layer capacitance is given as the product of $\mathrm{C}_{\mathrm{DL} \text {,Surface }}$ and area. Since this is the double layer constant for each electrode and the simplified model adds the two series capacitors, the capacitance is halved.

$$
\begin{gathered}
C_{G}=\frac{\epsilon_{0} \epsilon_{S}}{K_{C E L L}} \\
C_{D L}=0.5 \cdot A \cdot C_{D L, \text { surface }}=0.5 \cdot \mathrm{W} \cdot L \cdot N \cdot C_{D L \text {,surface }}
\end{gathered}
$$

As previously explained, at low frequency the double-layer capacitance - coating resistance branch dominates the impedance. It is assumed that the contact resistance is much smaller than the coating resistance and can therefore be ignored. The impedance around the low corner frequency given in equation 3.14, and solved for the corner frequency in equation 3.15, which is the frequency where the impedance of $R_{S}$ and $C_{D L}$ are equal.

$$
Z=R_{S}+\frac{1}{j \omega C_{D L}}=\frac{1+j \omega \mathrm{R}_{\mathrm{s}} C_{D L}}{j \omega C_{D L}}
$$




$$
f_{\text {LOW }}=\frac{1}{2 \pi R_{S} C_{D L}}
$$

By combining equation 3.13 and equation 3.15 , the low corner frequency can be determined as a function of the IDE geometry and the coating properties:

$$
f_{\text {LOW }}=\frac{\sigma_{S}}{\pi \cdot W \cdot L \cdot N \cdot C_{D L \text { surface }} \cdot K_{C E L L}}
$$

Around the high corner frequency, the principal contributors to the impedance are the geometry capacitance and the coating resistance. Since these elements are in parallel, the impedance is given in equation 3.17 with the corner frequency solved in equation 3.18, which is the frequency where the impedance of $\mathrm{RS}_{\mathrm{S}}$ and $\mathrm{C}_{\mathrm{G}}$ are equal.

$$
\begin{gathered}
Z=\frac{R_{S}}{1+j \omega R_{S} C_{G}} \\
f_{H I G H}=\frac{1}{2 \pi R_{S} C_{G}}
\end{gathered}
$$

Combining equations 3.8 and 3.12 with equation 3.18 shows that $f_{\mathrm{HIGH}}$ depends only on the coating properties. While there is more control over the low corner frequency, the high corner frequency can only be set by selecting a material with appropriate conductivity and dielectric.

$$
f_{H I G H}=\frac{\sigma_{S}}{2 \pi \epsilon_{0} \epsilon_{S}}
$$

During testing, when a sample is applied the molecules in the sample and the DI water will affect the equivalent dielectric and conductivity seen by the sensor. Due to the highly absorbent nature of the PVA-SbQ hydrogel, it will rapidly absorb any water on the sample, therefore the change in corner frequency is proportional to the change in the conductivity. The change in equivalent dielectric of the coating, water, and sample will 
inversely affect the high corner frequency. The change in the double layer interaction when

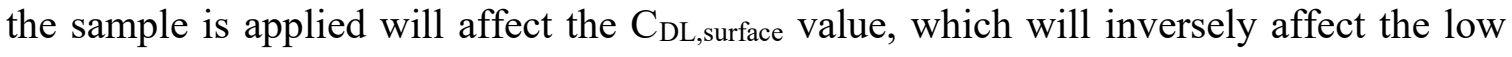
corner frequency. At the low concentration of analyte, $\sim 100 \mathrm{pg} / \mathrm{mL}$ maximum, and with the low amount of water on the sensor during the aerosolized tests, it is expected that the shifts in corner frequencies will be limited. Furthermore, the frequency shifts are used to detect the presence of a sample on the sensor, as the change in conductivity can be used to indicate the presence of a sample. A rapid change in the coating resistance can be easily detected and a microcontroller monitoring this resistance can use this parameter to detect the applied sample.

From testing of the aerosolized solutions, the corner low corner frequency would shift between $800 \mathrm{~Hz}$ to $3 \mathrm{kHz}$ and the high corner frequency up from $8 \mathrm{kHz}$ to $20 \mathrm{kHz}$, depending on the amount of water on the sensor. This was determined by analyzing the impedance data from various measurements and detecting where the three impedance regions begin. While this is an increase up to $\sim 3$ times the initial values, these corner frequencies are well within the scan range of the impedance analyzer and still create the three distinctive regions required to be able to extract the model parameters. By analyzing the complete model, the sensor is still functional even in the presence of these frequency shifts. The corner frequencies are still easily detectable, and the geometry capacitance, the main transconductance parameter, is not affected by the frequency shifts.

This was more of an issue in initial testing, as higher quantities of water and higher concentrations of analytes were used. The change in conductivity due the presence of the aqueous sample would increase both corner frequencies, leading to the requirement of higher frequency sweeps. 


\section{Chapter 4: Materials and Methods}

The fabrication process of the MIPs sensor consisted of two main components, the fabrication of the PDMS stamp, and the imprinting of the stamp onto the sensor. Once imprinted, the sensor was experimented first with aqueous solutions as a proof of concept and data collection, then the IDE was optimized, and the test setup was modified to use spiked aerosolized samples. Finally, a custom multiplexed sensor PCB was developed for small footprint, rapid response, point of care measurements.

\subsection{PDMS Stamps}

To create the MIP nanoimprints, the stamp must first be prepared. PDMS was selected as the stamp material as it is transparent and allows for UV to be used to induce polymerization, has no interaction with PVA-SbQ so it can be cleanly removed, and has good durability allowing the stamps to safely used for multiple sensors. PDMS was prepared by first mixing Sylgard 184 silicone elastomer (purchased from Dow Chemicals) and curing agent $1: 10$ for 1 hour at $60^{\circ} \mathrm{C}$, then cutting into the desired IDE structure size. The IDE sensors have a $5 \mathrm{~mm}$ by $5 \mathrm{~mm}$ sensing area, so the stamps were cut to $1 \mathrm{~cm} \mathrm{x} 1 \mathrm{~cm}$ squares. $1 \mathrm{mg}$ of 8 -isoprostane (purchased from Cayman Chemical) was dissolved in $1 \mathrm{ml}$

of ethanol, then an aliquot of $5 \mu 1$ was evaporated. The solute was resuspended in $50 \mu 1$ of DI water and a $25 \mu \mathrm{L}$ aliquot was subsequently dropped onto the PDMS film, which was dried for 45 minutes at $37^{\circ} \mathrm{C}$. This would attach the 8 -isoprostane to the stamp, which was confirmed through AFM imaging. In order to ensure the reusability of the stamps, AFM imaging was repeated after using the stamp over10 times. 
Both patterned and non-patterned PDMS stamps were tested to determine what method would have a better distribution of molecules on the surface. The patterned PDMS stamp is shown in Figure 16, consisted of $1 \mu \mathrm{m} \times 1 \mu \mathrm{m} \times 1 \mathrm{um}$ wells, spaced out by $1 \mu \mathrm{m}$. The purpose of the patterned stamp was to have better control over where the imprint sites would be, as well as create sites with high imprint density to theoretically improve the sensitivity of the device. It was observed that the deposited molecules would sink too deep into the wells and would not be contacting the PVA-SbQ during polymerization. For all sensors in this report, the stamps utilized were therefore non-patterned. To test the effects of the molecule packing density, additional stamps were produced by diluting the stock solution of $(1 \mathrm{mg} / \mathrm{ml})$. The different stamps from the different concentration of 8 isoprostane were labelled from $1 \mathrm{mg} / \mathrm{mL}$ for stamp $1,0.5 \mathrm{mg} / \mathrm{mL}$ for stamp 2, $0.25 \mathrm{mg} / \mathrm{mL}$ for stamp 3, and $0.125 \mathrm{mg} / \mathrm{mL}$ for stamp 4 . The stamps were stored in wafer boxes and kept on a wafer, as the solid support of the wafer would simplify the use of the AFM machine. The PDMS should not be left out if its case for extended periods of time unless it is inside a clean room, as it can rapidly collect dust and particulates on the surface. The PDMS stamps were replaced if these particulates were noticed, as they can prevent the template from contacting the polymer.
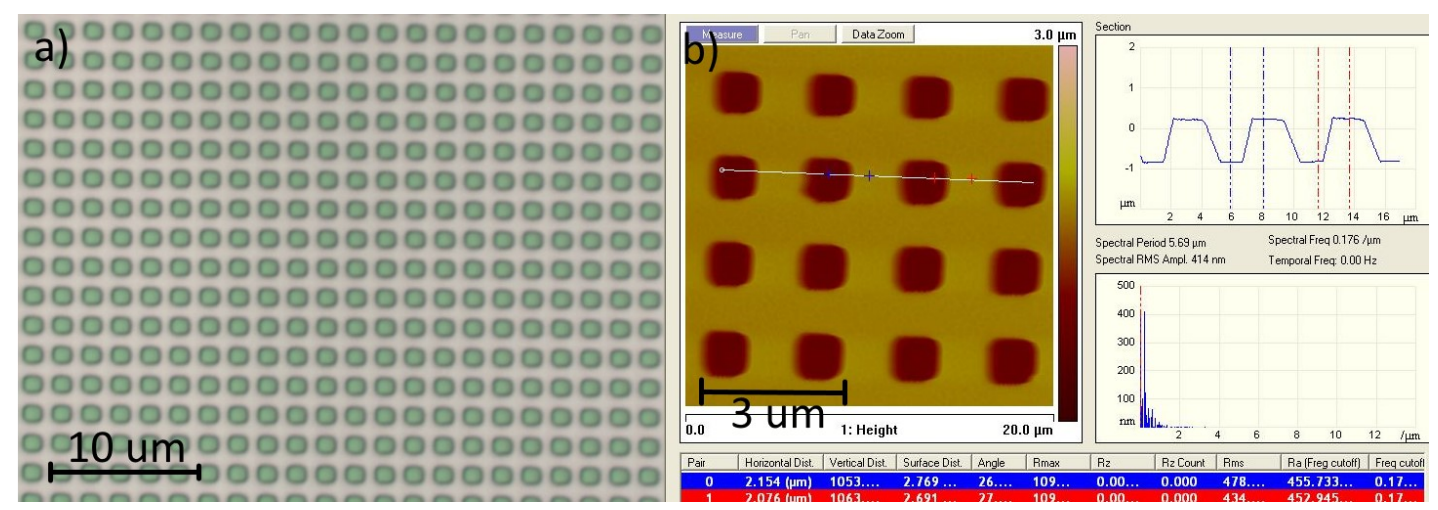

Figure 16 - (a) Patterned PDMS Stamps (b) AFM of patterned PDMS Stamp 
To improve the distribution of the template on the stamp, after dropping the 8isoprostane on the stamp, the stamp was spun at 100 to $1000 \mathrm{rpm}$. It was noticed that even at $100 \mathrm{rpm}$ for 3 seconds, the surface energy (the excess energy on the surface that determines the adhesion properties) on the PDMS stamp was too low for the 8-isoprostane solution to spread out properly. Therefore, this step was removed from the final stamp preparation method.

\subsection{IDE Parameters}

The IDEs used in this experiment were micropatterned using standard photolithography of $200 \mathrm{~nm}$ chrome layer, deposited on passivated silicon substrates. The IDE dimensions were set utilizing the equations outlined in section 3.3. To optimize the IDEs, the PVA-SbQ film conductivity and dielectric must be known for the high corner frequency, and the $\mathrm{C}_{\mathrm{DL} \text {,surface }}$ characteristic value is also required for the low corner frequency. These parameters are dependant on the thickness of the PVA-SbQ layer and the ratio of water to PVA-SbQ and were determined experimentally. Averaging the measurements from 10 sensors, the conductivity was found to be approximately $76 \mathrm{uS} / \mathrm{m}$, with an average relative dielectric constant of 75 . Using equation 3.19, the high corner frequency is around $16 \mathrm{kHz}$. The $\mathrm{C}_{\mathrm{DL} \text {,surface }}$ characteristic value was found to around 1.3 $\mathrm{F} / \mathrm{cm}^{2}$.

To simplify the analysis of the low corner frequency the IDE sensor is set to a square area so that $\mathrm{L}=\mathrm{N} \cdot(\mathrm{W}+\mathrm{S})$ as shown in Figure 8 . The sensitivity of the sensor increases with the contact area between the electrodes and the sensing coating, which Ibrahim et al. have demonstrated to be optimal when $\frac{W}{S}=0.66$ [79]. With the IDE finger 
ratios fixed, the cell constant vs N relationship is an exponential decay. Since the double layer capacitance is inherently larger than the geometry capacitance, the cell constant can be set to small number to ensure that the region 1 impedance will not dominate too much of the impedance spectrum. Above 16 fingers, adding more fingers has minimal effect of the cell constant, so a range of fingers between 16 and 20 is optimal.

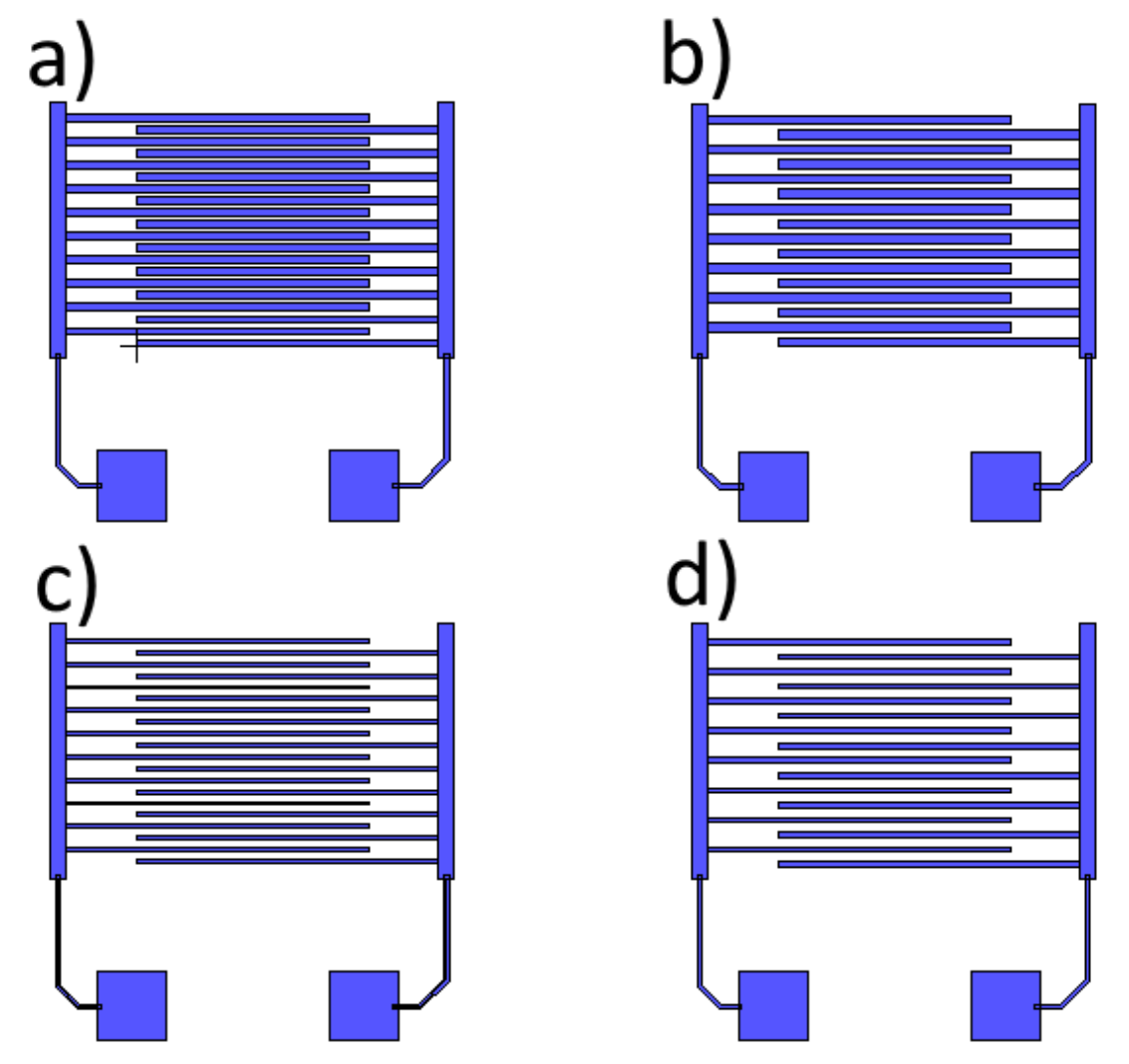

Figure 17 - Optimized IDE Layouts used for Aerosolized Sample Testing

The final sensing area was set to $5 \mathrm{~mm} \times 5 \mathrm{~mm}$ to have a small-footprint sensor. To have a square sensing area using the equations above, this sets the finger length to $5 \mathrm{~mm}$. The number of fingers was set to 20 , giving a spacing of $102 \mu \mathrm{m}$ and a width of $153 \mu \mathrm{m}$; using equation 3.16 gives a low corner frequency around $1.3 \mathrm{kHz}$. Evaluating the cell constant in MATLAB gives a value of $18.2 \mathrm{~m}^{-1}$. For evaluation purposes, 3 additional sensors with different finger numbers and dimensions but the same sensing area (constant 
length) were constructed. These two corner frequencies were each approximately a third of the logarithmic frequency sweep. A modified version of equation 3.12 is used to provide a more complete estimate of the initial geometry capacitance which incorporates the capacitance due the electric field between the sides of the fingers, while the earlier equation assumed the thickness of the IDE fingers could be ignored [80]. The additional $\mathrm{T}$ term is the thickness of the electrodes, which is $200 \mathrm{~nm}$. As the fingers are completely covered by the coating, the dielectric constant between them is due to the dielectric constant of the coating [81]. Table 2 shows the dimensions of the four IDEs used and the estimated nominal capacitance, and Figure 17 shows the layout for the four IDEs.

$$
C_{G, \text { nom }}=L(N-1) \epsilon_{0} \epsilon_{S}\left(\frac{1}{2} \frac{K\left(\sqrt{1-k^{2}}\right)}{K(k)}+\frac{2 T}{S}\right)
$$

Table 2 - Optimized IDE Dimensions

\begin{tabular}{|c|c|c|c|c|c|}
\hline Sensor & $\mathrm{N}$ & W & $\mathrm{S}$ & $\mathrm{L}$ & $\mathrm{C}_{\mathrm{G}, \mathrm{nom}}$ \\
\hline A & 20 & $153 \mu \mathrm{m}$ & $102 \mu \mathrm{m}$ & \multirow[t]{4}{*}{$5 \mathrm{~mm}$} & $36.7 \mathrm{pF}$ \\
\hline B & 16 & $192 \mu \mathrm{m}$ & $128 \mu \mathrm{m}$ & & $28.9 \mathrm{pF}$ \\
\hline C & 20 & $83 \mu \mathrm{m}$ & $167 \mu \mathrm{m}$ & & $26.6 \mathrm{pF}$ \\
\hline $\mathrm{D}$ & 16 & $104 \mu \mathrm{m}$ & $208 \mu \mathrm{m}$ & & $19.6 \mathrm{pF}$ \\
\hline
\end{tabular}

\subsection{Imprinting}

The imprinting process is shown in Figure 18. From experimental tests it was determined that $10 \mu \mathrm{L}$ of PVA-SbQ solution (purchase from Polysciences Inc.) could be used to obtain an appropriate film thickness of $250 \mathrm{~nm}$. To ensure the hydrogel would only form over the IDE structure and not the contact bars or contact pads, everything except the $5 \mathrm{~mm} \times 5 \mathrm{~mm}$ sensing was taped over. As the model only expects contact between the electrode fingers and the sensing material, it was important to ensure it would no spill over 
onto the contact pads. The solution was $13.3 \%$ PVA-SbQ with $4.1 \mathrm{~mol} \%$ being the SbQ and was dropped onto the surface then any bubbles were carefully removed. The IDE was then spun in a centrifuge at $2000 \mathrm{rpm}$ for 3 seconds to achieve the desired thickness. The tape was removed, and a stamp was put on the coating for imprinting. A glass slide is then placed on top to clamp down the stamp. This was done in a dark room to avoid having any UV exposure that would start to polymerize the solution before the stamp is placed. If a dark room is not accessible, the stamp must be placed over the PVA-SbQ solution immediately. The glass slide weights just enough to apply some pressure on the solution and ensure good contact between the templates and the PVA-SbQ but not enough to spread out the solution further. The sensor was then exposed to a UV lamp with a $365 \mathrm{~nm}$ wavelength for 30 minutes at room temperature. The PDMS will stick to the glass since it is hydrophobic and won't stick to the polymer, so when the glass slide is lifted the stamp comes off with it. The sensor was then be washed with DI water to remove away any unpolymerized PVA-SbQ which is still soluble in water while the polymerized PVA-SbQ has become insoluble in water. Before the first measurement an ethanol wash was done to dissolve any 8-isoprotstane that may have been left on the sensor after polymerization, although as shown in the results section no noticeable loss of template on the stamp was detected. 


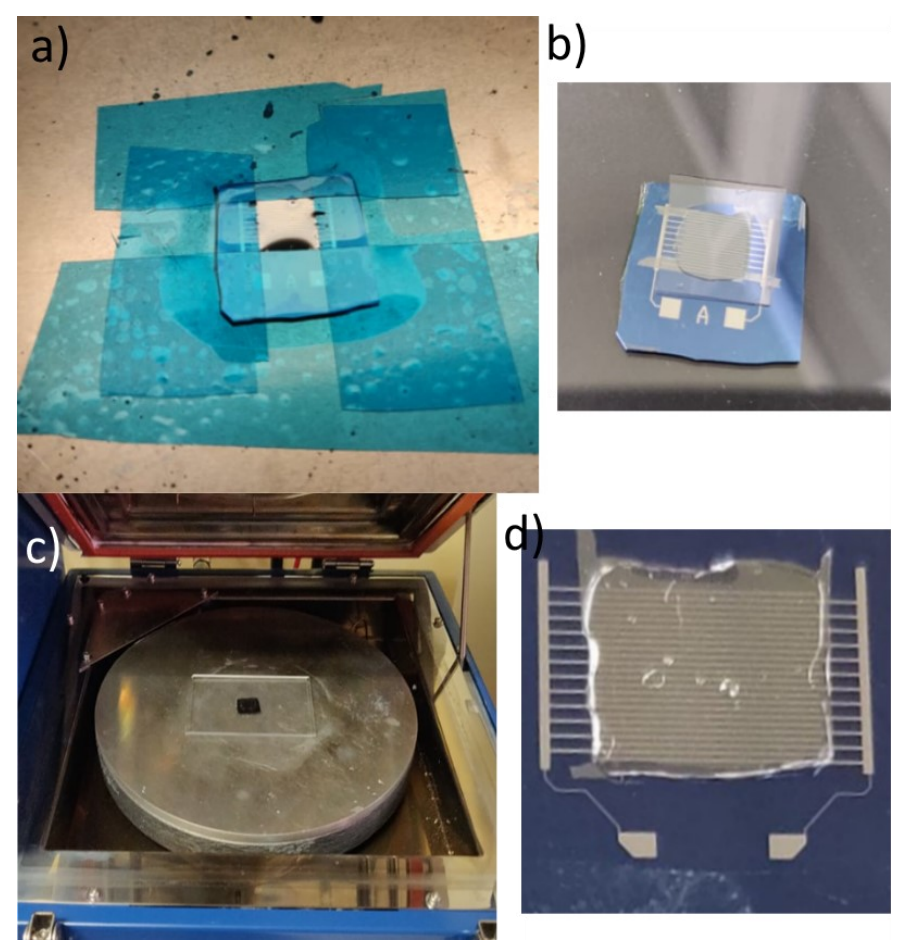

Figure 18 - (a) Protected IDE with deposited PVA-SbQ (b) IDE spun, tape removed, and stamp placed (c) IDE clamped with glass slide for UV exposure (d) Final imprinted Sensor

\subsection{Data Acquisition}

Before all sets of measurements, the impedance analyzer was recalibrated using the Agilent 42941A impedance probe. The impedance is then logarithmically swept across the desired the frequency range, which was $40 \mathrm{~Hz}$ to $1 \mathrm{MHz}$ for initial aqueous sample testing, and then reduced to $40 \mathrm{~Hz}$ to $100 \mathrm{kHz}$ for the optimized sensors with aerosolized samples. The lower limit of the impedance analyzer is $40 \mathrm{~Hz}$, and since commercial EIS chips are limited to $100 \mathrm{kHz}$, we selected these values for the optimized sensors. The Agilent 4294A impedance analyzer was set to capture the impedance phase and magnitude, which would be exported to an excel spreadsheet.

The probes of the impedance analyzer were connected to a probe station to allow for finer control of the probe contact. For the spikes aerosolized tests shown in Figure 19, 
an aerosolization chamber was prepared by curing PDMS around a $2 \mathrm{~mm}$ diameter polymer tube which doubles as the air intake channel. A small hole was drilled on top to allow for the sample to be mixed with the elevated pressure air flow (exhaled breath) used for aerosolization in the sensing chamber. For an exhaled breath test, as a subject blows into the intake channel some water from the test sample micro-well is aerosolized and transferred into the test chamber, which will help the particles in the exhaled breath to spread out more. To simulate this, the solution was already spiked with the sample, and the process is reversed. As pressured air was blown into the channel a small amount of the spiked solution would be sucked out of its container and would be blown onto the sensing surface.

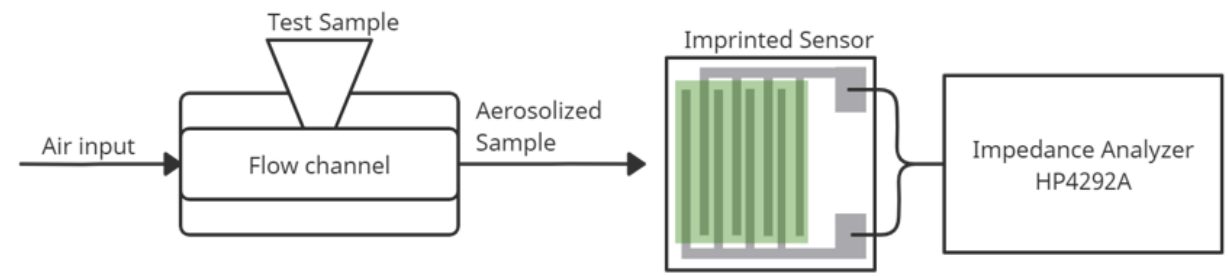

Figure 19 - Aerosolized Sample Setup

After connecting the probes to the sensor, a series of baseline measurements are taken to model the device characteristics without any sample. To analyze the response time of the sensor, measurements were taken every 10 seconds for 2 minutes once the sample was introduced onto the sensor. The response is the time required between the sample application and the output signal stabilizing. Once the sensor settling time was discovered, samples required only one measurement taken 60 seconds after the sample was applied. After a measurement set was complete, the sensor was washed with ethanol to dissolve any bound template. The ethanol was then gently blown off using pressurized air, 
then placed in an oven at $35^{\circ} \mathrm{C}$ for 1 minute to dry. The drying time is critical as leaving the sensor in the oven for too long will over dry the hydrogel which will start to peel-off from the sensor surface.

To test to the solution selectivity, the measurements were taken with both an imprinted and a non-imprinted sensor with DI water solutions containing no sample, 8isoprostane, Cortisol, Cortisone, and Progesterone purchased from Sigma Aldrich. The additional three molecules are all molecules that are also found in exhaled breath in similar concentrations to 8-isoprostane and have similar molecular masses to 8-isoprostane [82]. The chemical formulas and molar masses of the molecules are shown in Table 3 to demonstrate their similarity. Due to the different shapes of the molecules, mostly in the locations of any -OH functional groups, the imprinted cavities should only be able to capture 8-isoprostane. The solutions were serially diluted from a starting stock solution of $1 \mu \mathrm{g} / \mathrm{mL}$ by pipetting $5 \mu \mathrm{L}$ of the solution into a test tube and adding an extra $45 \mu \mathrm{L}$ of DI water. This was repeated 9 times for each solution to reach solutions with $1 \mathrm{pg} / \mathrm{mL}$. The stock solution would be frozen for reuse, and the serial dilution would be redone if the existing diluted solutions were more than a few days old.

Table 3 - Molecules for Experimentation

\begin{tabular}{|l|l|l|}
\hline \multicolumn{1}{|c|}{ Molecule } & Chemical Formula & \multicolumn{1}{|c|}{$\begin{array}{c}\text { Molar Mass } \\
(\mathbf{g} / \mathbf{m o l})\end{array}$} \\
\hline 8-isoprostane & $\mathrm{C}_{20} \mathrm{H}_{34} \mathrm{O}_{5}$ & 354.5 \\
\hline Cortisol & $\mathrm{C}_{21} \mathrm{H}_{30} \mathrm{O}_{5}$ & 362.46 \\
\hline Cortisone & $\mathrm{C}_{21} \mathrm{H}_{28} \mathrm{O}_{5}$ & 360.45 \\
\hline Progesterone & $\mathrm{C}_{21} \mathrm{H}_{30} \mathrm{O}_{2}$ & 314.5 \\
\hline
\end{tabular}


For the aqueous solution experiments, the liquid solutions were dropped on the sensor surface using a pipette. $5 \mu \mathrm{L}$ of the solution was used, and the solution was left on the sensor for 2 minutes before being removed manually. The aqueous solution was not left on for any longer as there was a risk of water getting trapped underneath the polymer layer along the edges, causing some undesired lift-off. For the aerosolized sample testing, the same volume of sample was placed in the sample holder micro-well, and low-pressure air (around 1 psi) was blown through the aerosolization chamber until all $5 \mu \mathrm{L}$ of the sample was on the sensor. For aqueous samples, to ensure the dropped sample would remain within the sensing area, a PMDS well was created by punching a $5 \mathrm{~mm}$ diameter hole into PDMS. A glass slide would then be placed over to enclose the sample. This setup used pre-existing IDEs that were not optimized for this application, however, it was sufficient for model building and characterization of the materials, as well as proving the concept of the sensor. This was done for concentrations of $1 \times 10^{4} \mathrm{pg} / \mathrm{mL}$ to $1 \times 10^{9} \mathrm{pg} / \mathrm{mL}$ as it would be simpler to test the functionality of the sensor at very high concentration and would also be used to detect the saturation limit of the sensor. The setup is shown in Figure 20. 


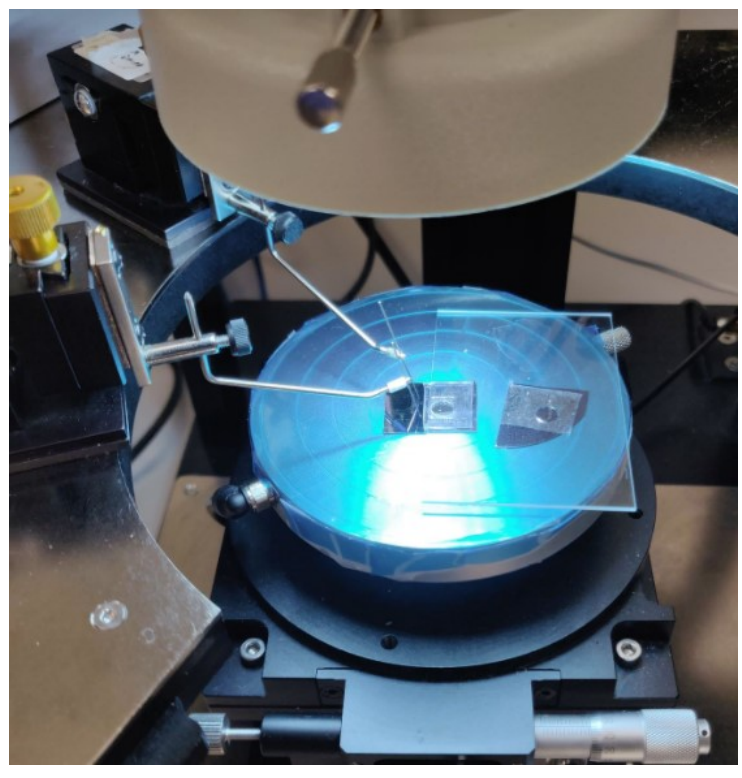

Figure 20 - Probe Station Setup

After the IDE optimization was complete, the test setup was modified to use the spiked aerosolization method. The red tube in Figure 21 is the pressurized air intake going into the PDMS aerosolization chamber, and a small test tube with a small hole on the bottom was used to hold the sample (died in red in Figure 21). The diameter of the hole of the test tube was small enough that no sample would flow out but large enough that small amounts could flow in the presence of pressurized air. The impedance analyzer probes were connected to other probes in order to contact the circuit. 


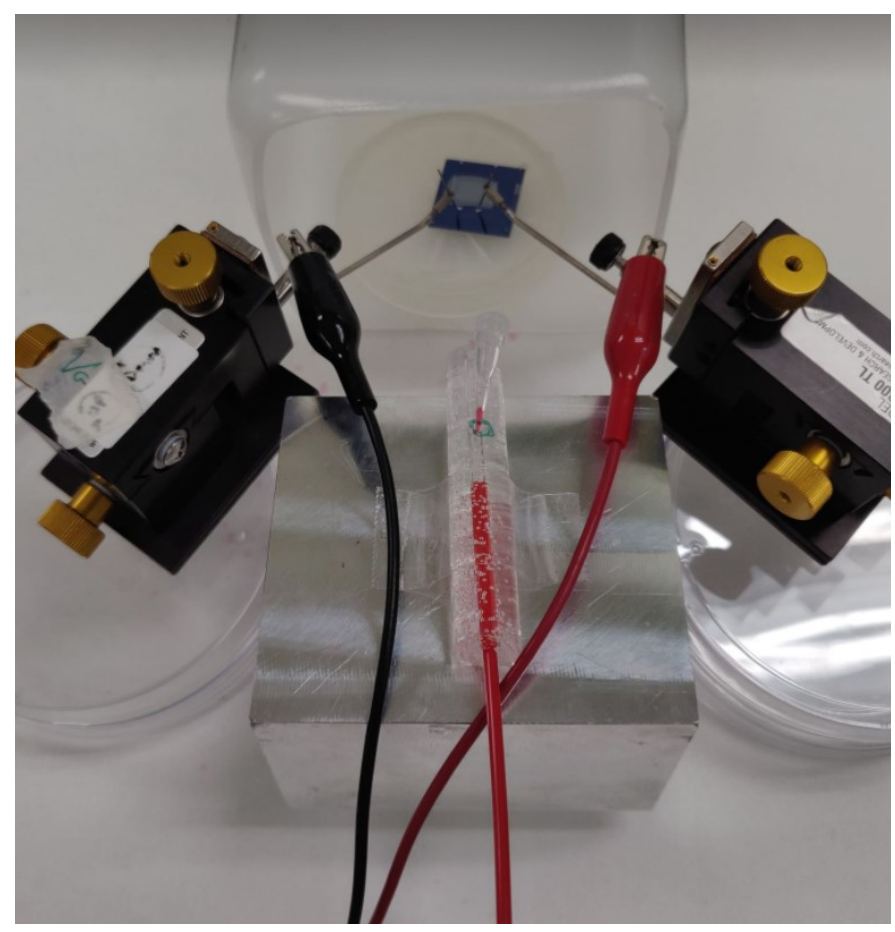

Figure 21 - Aerosolized Sample Setup

\subsection{Parameter Extraction}

Once all the measured data was exported to an excel spreadsheet, the data was then reformatted using a excel visual basic script to first convert the impedance phase and magnitude to the real and imaginary components of the impedance using equations 4.2 and 4.3. The script would also generate the Nyquist plots for all measurements for rapid comparison and analysis. The rest of the data analysis was performed using MATLAB which would import the real and imagery components of the impedance.

$$
\begin{aligned}
& Z^{\prime}=|Z| \cos (\theta) \\
& Z^{\prime \prime}=|Z| \sin (\theta)
\end{aligned}
$$


The Nyquist plot is a plot of the negative imaginary impedance against the real impedance. On a Nyquist plot, a resistor is a single point along the $\mathrm{x}$-axis since it has no imaginary component and a fixed real component. A capacitor is a line along the y-axis since it has no real component, and the imaginary component will change depending on the frequency. For the 4-element equivalent circuit model, the Nyquist plot has the shape of a semi-circle with a tail on the end. The frequency shifts from low to high across the $\mathrm{x}-$ axis of the graph. The tail is the low frequency component of the impedance, which is the double layer capacitance. The angle of the tail relates to the ideality factor of the constant phase element used to represents the double layer capacitance, an $\alpha$ of 1 (ideal capacitor) would mean the angle of the tail would be $90^{\circ}$, going straight up. An $\alpha$ of 0.5 would mean a tail angle of $45^{\circ}$. Since the relationship between the tail angle and $\alpha$ is $\alpha=\frac{\text { tail angle }}{90^{\circ}}$, the ideality factor can be easily extracted. Since the impedance is dominated by the double layer capacitance in the tail since it is the low frequency component, using equation 3.1 at low frequency will give the value of the double layer capacitance. Due to the presence of noise, the average of a few data points at low frequency is used.

The two resistors and the capacitor form a semicircle, which can used to determine the rest of the parameters. As shown in Figure 22, which is representative of the typical Nyquist plot from the measured data, the $\mathrm{x}$-intercepts will give $\mathrm{R}_{\mathrm{C}}$ and $\mathrm{R}_{\mathrm{C}}+\mathrm{R}_{\mathrm{S}}$ while the frequency at the peak of the semi-circle is required to calculate $C_{G}[71]$.

$$
f_{\text {peak }}=\frac{1}{2 \pi C_{G} R_{S}}
$$




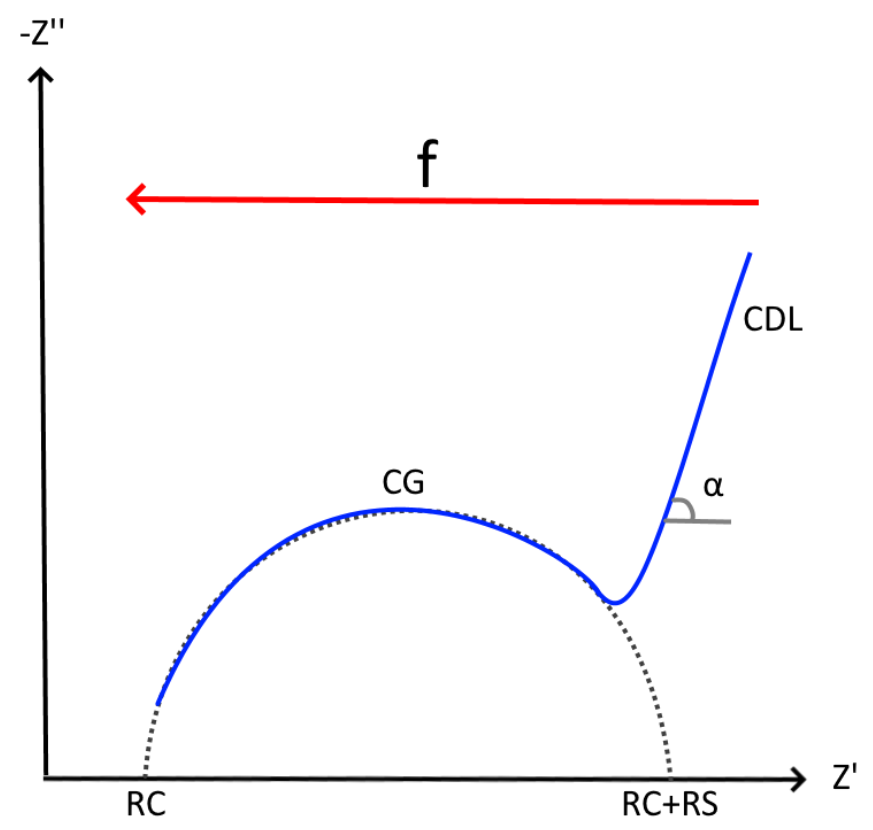

Figure 22 - Representative Nyquist plot of impedance

Once the data is exported to MATLAB, the script first attempts to estimate the quality of the input data by quickly evaluating all the data points comparing it to Figure 22. First, the script looks for a corner point that indicates the point between the tail and the semicircle, and then makes sure that there is enough of a semi-circle present to calculate its radius and intercepts (i.e., after the corner, the data must reach a peak and start to decrease). Then, the program checks if there is any data beyond the end the semicircle which can be ignored. At frequencies much higher than high corner frequency, the model may become inaccurate, as additional elements whose impedance only becomes significant may appear. If the criteria for an ideal impedance measurement have been met, the script will then do the parameter extraction. If no clear corner between the semicircle and the tail is detected, the data is scanned for an 'unclear corner,' as in the tail 
can still be distinguished by the semicircle is not clear, which can occur when the two corner frequencies of the sensor have shifted too close together such as in Figure 23.

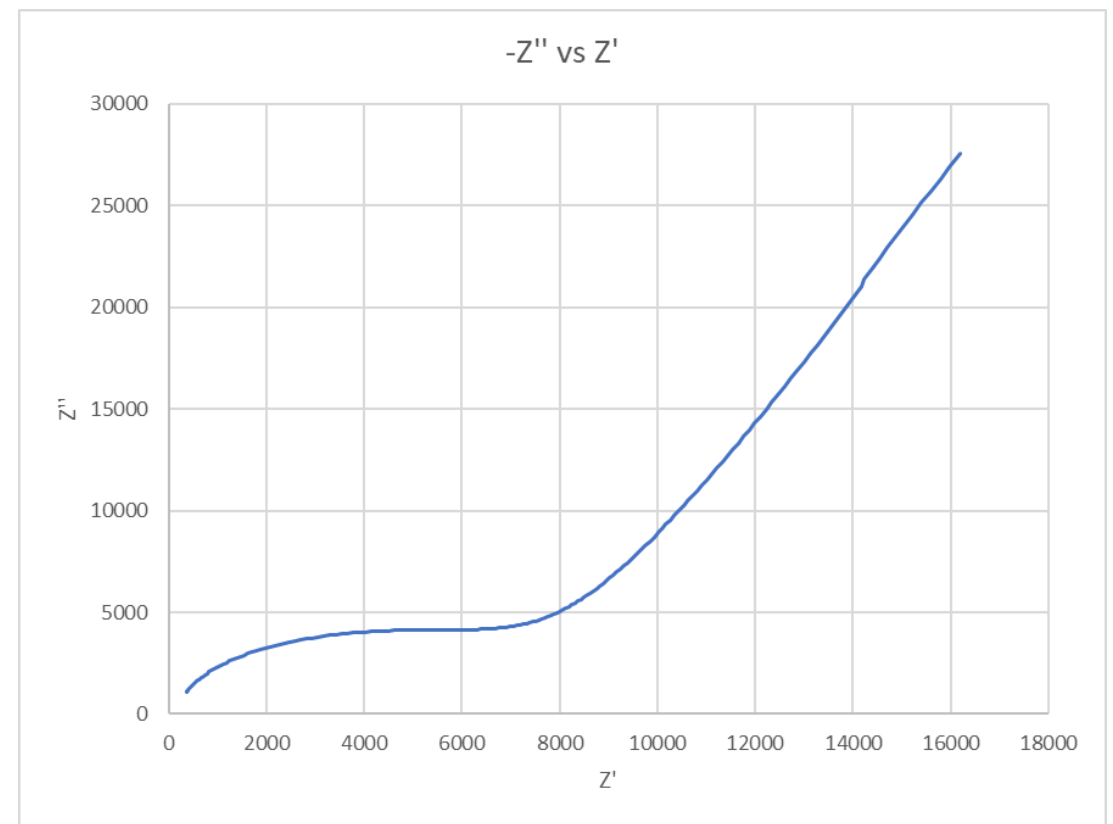

Figure 23 - Measured impedance example of no clear corner

While there is no clear corner between the two parts of the Nyquist plot, there is a plateau than can be detected if the shift in corner frequencies is not large. With a $100 \mathrm{kHz}$ scan, if the two corner frequencies are less than an order of magnitude apart, there may not be enough distinction to determine when the three impedance regions start. The issue with no clear peak of the semi-circle is that the frequency at the peak is required to be able to calculate the model parameters, and they can only be estimated in these cases. If the plateau is detected, it is considered to be the peak of the semicircle. If this assumption is close to actual value, the model can still extract the parameters with high accuracy. This is verified by comparing the measured impedance data to the model impedance data. As there is no clear point where the semi-circle ends the tail begins, only the first few points of the tail are used to estimate the double layer capacitance ideality. If no plateau is detected, the 
analysis is still attempted as a reasonably close approximation is still achievable, however this will likely fail as the corner frequencies are unknown. Finally, any high frequency data after the semi-circle can be assumed to noise and is removed from the data set.

With the data properly segmented, the slope of the tail is calculated using a linear fit from the data points in the tail. Equation 3.2 is then used over half the data points in the tail then averaged to estimate $\mathrm{C}_{\mathrm{DL}}$. Since the geometry capacitance is essentially ideal, the semicircle data is then fitted to a circular equation using the MATLAB circlefit () function. As expected, the center point has a y value of $\sim 0$ for all measurements. $\mathrm{R}_{\mathrm{C}}$ is acquired by subtracting the radius from the $\mathrm{x}$ center point and $\mathrm{R}_{\mathrm{S}}$ by adding the radius to the $\mathrm{x}$ center point then subtracting the value of $R_{C}$. The datapoint closest to the calculated circle peak is used with equation 4.4 to obtain the value of $\mathrm{C}_{\mathrm{G}}$. If the data cannot be fit to a circle, the data is assumed to be invalid.

Theoretically, only the few calculations outline in this section are required to extract the model parameters, however there are several factors that affect the extraction, which led to expanding the algorithm to attempt to improve the accuracy of the results. Firstly, outlier data points or a disproportionate spread of data points can lead to a skewed calculation of the semi-circle properties. Secondly, the peak data point that is used in the calculation of the geometry capacitance is only the highest measured peak, and the exact peak and its frequency may vary by a large amount for non-ideal measurements. Finally, the angle of tail will start to vary is it approaches the corner point, and if too many of those data points are included the ideality factor of the CPE may need to be adjusted. For the measurements that are "ideal" under the parameters outlined above, the optimization 
process is not required, however for non-ideal measurements the optimization process was shown to improve the accuracy of the model results by up to $25 \%$.

After the first pass the model parameters are input into equation 3.4 to produce a measured vs theoretical Nyquist and impedance plots. The accuracy of the model is determined by analyzing the percentage error between the theoretical and measured impedance magnitude vs frequency and impedance phase vs frequency plots. If the average percentage error is too large, the model is then refit. The first check is to recheck the values of $R_{C}$ and $R_{S}$, if in the Nyquist plots the corner and end points do not match up, $R_{C}$ and $R_{S}$ are increased or decreased by the same difference between the measured and calculated end/corner points. If only one side of the semicircle is known, only the closest data point is used. A change in $\mathrm{R}_{\mathrm{S}}$ means that $\mathrm{C}_{\mathrm{G}}$ will need to be recalculated, and the frequency at the peak is then estimated as the average frequency using the closest data point on each side of the peak. The double layer capacitance is then evaluated by comparing the length and angle of tail. If the angle or length has too large of a difference, the corresponding parameter is then modified by the difference between the angles and the value of $\mathrm{C}_{\mathrm{DL}}$ is recalculated. This is repeated a maximum of 10 times (selected arbitrarily to keep the modeling from taking too long) or until the percentage error has fallen to an acceptable value. 


\section{Chapter 5: Experimental Results and Discussion}

\subsection{Physical and Chemical Characterization of the MIP sensing Layer}

The surface morphology analysis of the stamps and sensor were performed on an Atomic Force Microscope (AFM) to confirm that the molecule was stamped onto the PDMS substrate and to observe the effect of concentration on packing density. Figure 24 shows the surface of the of the stamps with different concentrations of 8 -isoporstane. The 8-isoprostane remains on the stamp surface due to PDMS being highly hydrophobic. It is clear that stamps 3 and $4(0.25 \mathrm{mg} / \mathrm{mL}$ and $0.125 \mathrm{mg} / \mathrm{mL})$ do not have enough bound 8isoprostane to be used in a high sensitivity sensor. The stamp templated density was tested to determine the density where there was a high concentration of template without it being so saturated that it cannot form proper connections to the polymer during imprinting. From the imaging for stamps 1 and 2, the bound 8-isoprostane is spread out in small 4-5 nm groups with some large $\sim 20 \mathrm{~nm}$ clumps. The large $20 \mathrm{~nm}$ groups will form high concentration binding areas leading to areas of large dielectric changes, while the smaller binding areas will help ensure the sensed molecules is spread out over the sensing area.
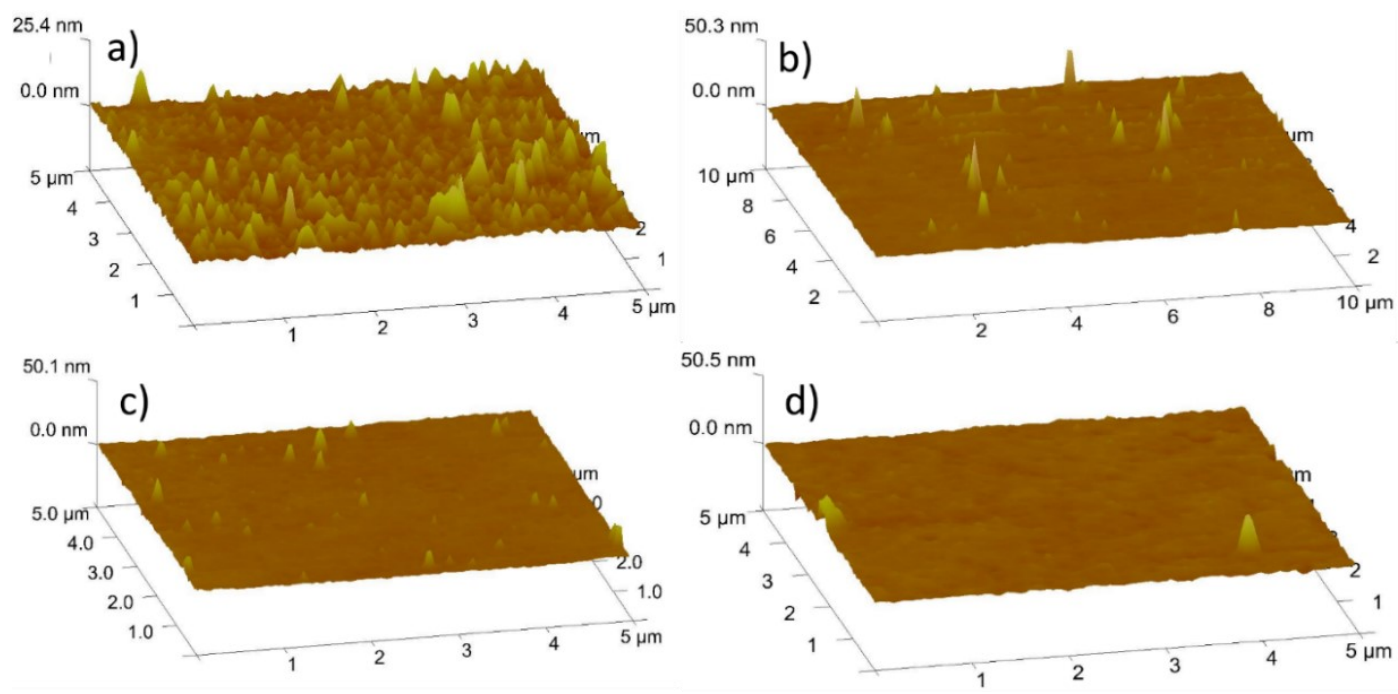

Figure 24 - Comparison of Stamps 1-4 (a-d) using AFM 
The surface roughens is shown in Figure 25 along with a picture of the stamps, including a stamp (stamp 0) with no template to be used for the non-imprinted sensors. While both stamps 1 and 2 were initially used create sensors to compare their results, stamp 1 was finally chosen for all subsequent sensors due to having higher sensitivity due to having a higher concertation of the template. Figure 34 in the experimental results demonstrates the difference in performance between to two sensors.

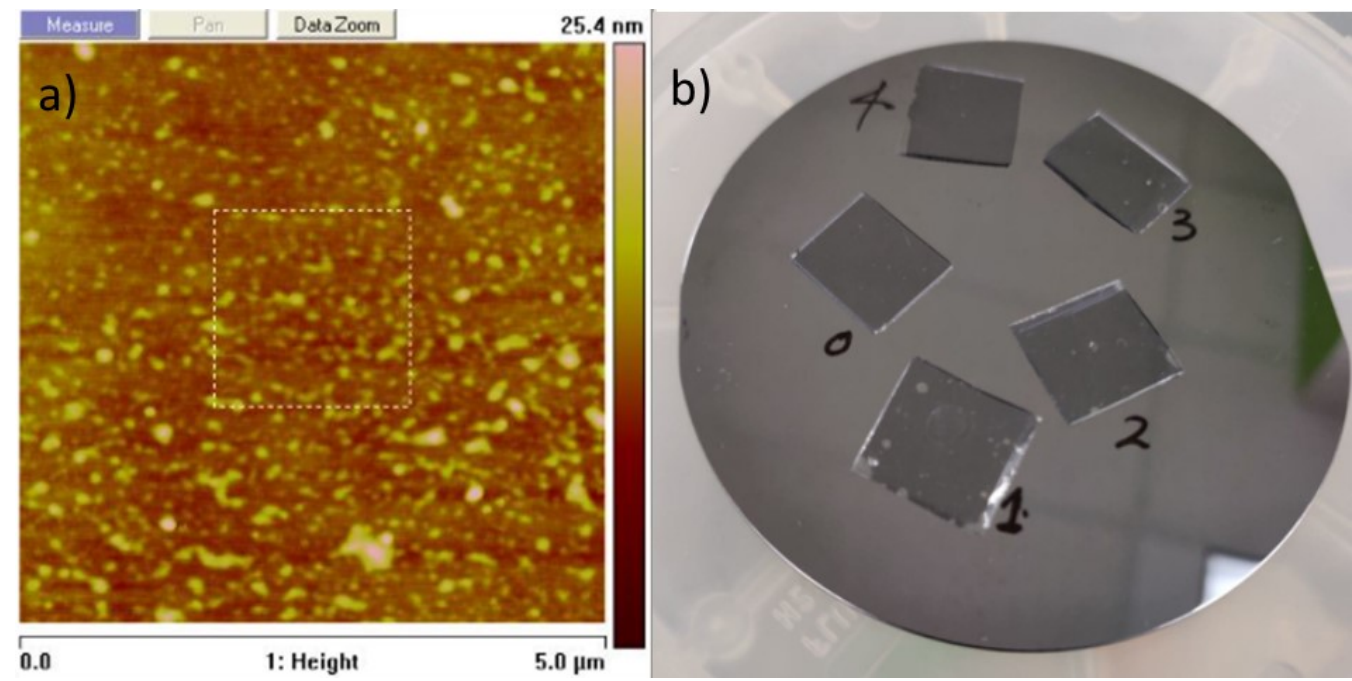

Figure 25 - (a) AFM of Stamp 1 surface roughness (b) Image of Stamps 0-4

One of the many benefits of the surface imprinted MIPs sensors is the reusability of the stamps, which is due to the fact the templates remain on the stamp after use. After using the stamps $>10$ times, the surface of stamp 4 was reanalyzed with the AFM, shown in Figure 26, demonstration minimal decrease in packing density. Since there are no markings on the stamp the area that was scanned was different, however the large $20 \mathrm{~nm}$ spikes are still present, however the density of the smaller particles has decreased slightly. 

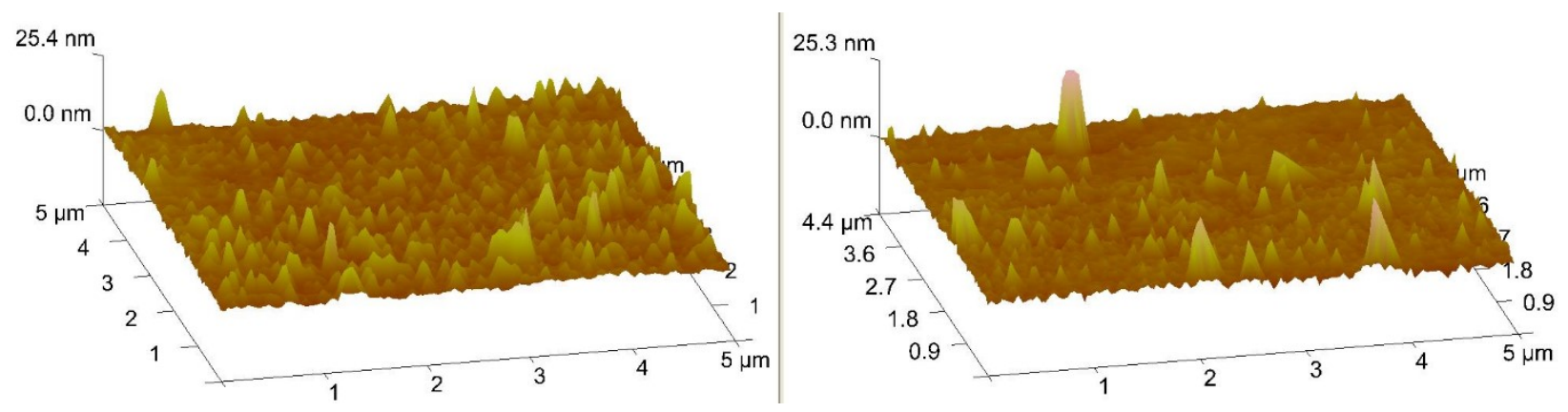

Figure 26 - Stamp Reusability, AFM image of Stamp 4 after creating the stamp and after 12 uses

As outlined in section 4.2, 4 different IDEs were prepared for testing. Although all 4 had similar corner low corner frequencies (high corner frequency is independent of the IDE), the sensitivity of sensors A and B were higher due to the larger electrode-coating interface area. Sensors A and B demonstrated the same sensitivity as they have the same area, however sensor A has a larger absolute change in capacitance due to having a smaller cell constant, making sensor A the preferable sensor for this application. The comparative nominal impedance of the four sensors is demonstrated in Figure 31, and the comparative capacitance change at $100 \mathrm{pg} / \mathrm{mL}$ between the four sensors is demonstrated in Figure 32 . Sensor $\mathrm{A}$ also has the largest $\mathrm{C}_{\mathrm{G}, \text { nom }}$ which means it is the least susceptible to parasitic capacitance.

The cross section of the sensor after the imprinting process was taken using SEM imaging shown in Figure 27. The passivation of the sensor surface is shown by the $2 \mu \mathrm{m}$ silicon dioxide layer over the substrate. The passivation process ensures no unwanted interaction between the polymer sensing layer and the substrate, as well as protect against oxidation due to the substrate reacting with air. The imaging also confirms the thickness of PVA-SbQ polymer layer to be approximately $250 \mathrm{~nm}$ from the imaging from multiple sensors. 

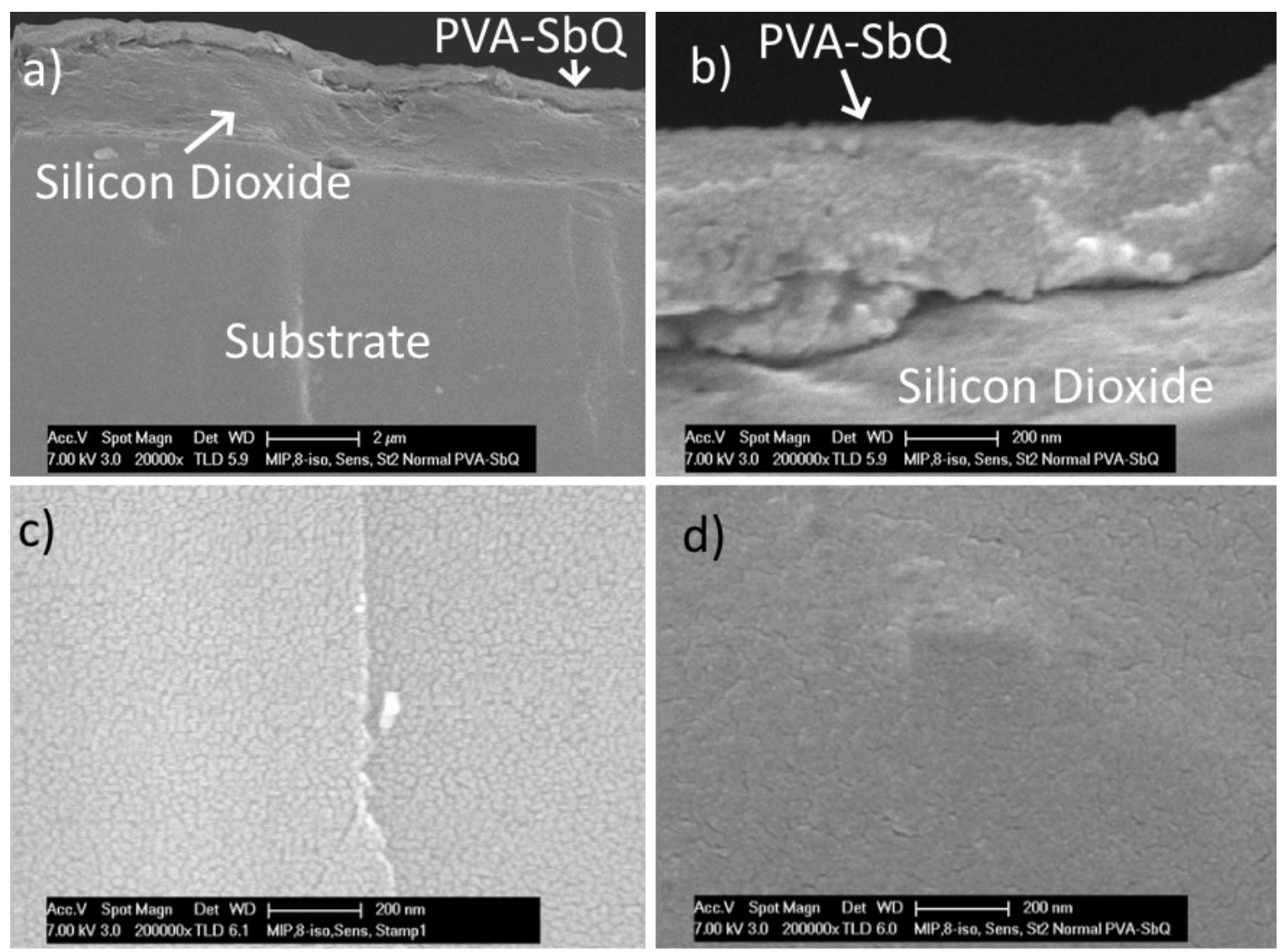

Figure 27 - (a) Cross section of sensor (b) Close up of polymer layer (c) Stamp 1 surface (d) Stamp 2 surface

The surface of the imprinted polymer was also examined through SEM imaging for sensors created using stamps 1 and 2. As shown by stamp AFM images, sensors that have been made using stamp 1 have a better spread of imprinted sites along its surface, while the surface for stamp 2 is mostly consistent of the binding sites created by the large concentration areas of 8 -isoprotane. The sensing mechanism can be seen in these images, as the imprinted areas should easily trap any 8-isoprotane molecules that reach the surface, while other molecules are much less likely to connect to the imprints and will remain just on the surface. 
Figure 28 shows that chemical characterization was performed on a Fourier transform infrared spectrophotometer (FTIR) showing the degree of acetylation in the PVA at 1725 $\mathrm{cm}^{-1}$. The $2946 \mathrm{~cm}^{-1}$ wavenumber shows $\mathrm{C}-\mathrm{H}$ stretch, $1246 \mathrm{~cm}^{-1}$ is due to $\mathrm{C}=\mathrm{C}$ bonding, while the absorbance peak at $3315 \mathrm{~cm}^{-1}$ is due to hydrogen bonding. The FTIR response shows no change in the peak locations between the partially polymerized PVA-SbQ, the imprinted polymerized PVA-SbQ, and the imprinted polymerized PVA-SbQ after exposure to $5 \mu \mathrm{L}$ of $100 \mathrm{pg} / \mathrm{mL}$ of 8 -isoprostane. Since the PVA-SbQ solution is already mostly polymerized, there is essentially no change in the absorbance after polymerization, especially with the very low concentration of the cross-linker. There is no shift in the absorbance peaks after exposure to the target molecule, indicating that the sensor functionalizing to the target molecule in the imprinted nanocavities is of purely steric nature. This was expected due to the generic preparation materials and methods for the presented sensor. Although no chemical interaction is present, there is a noticeable decrease in magnitude of the absorbance peak especially at the $3315 \mathrm{~cm}^{-1}$ wavenumber, indicating some possible changes in the hydrogen bonding, which was previously determined to be how the 8-isoprostane interacts with the nanocavities. 


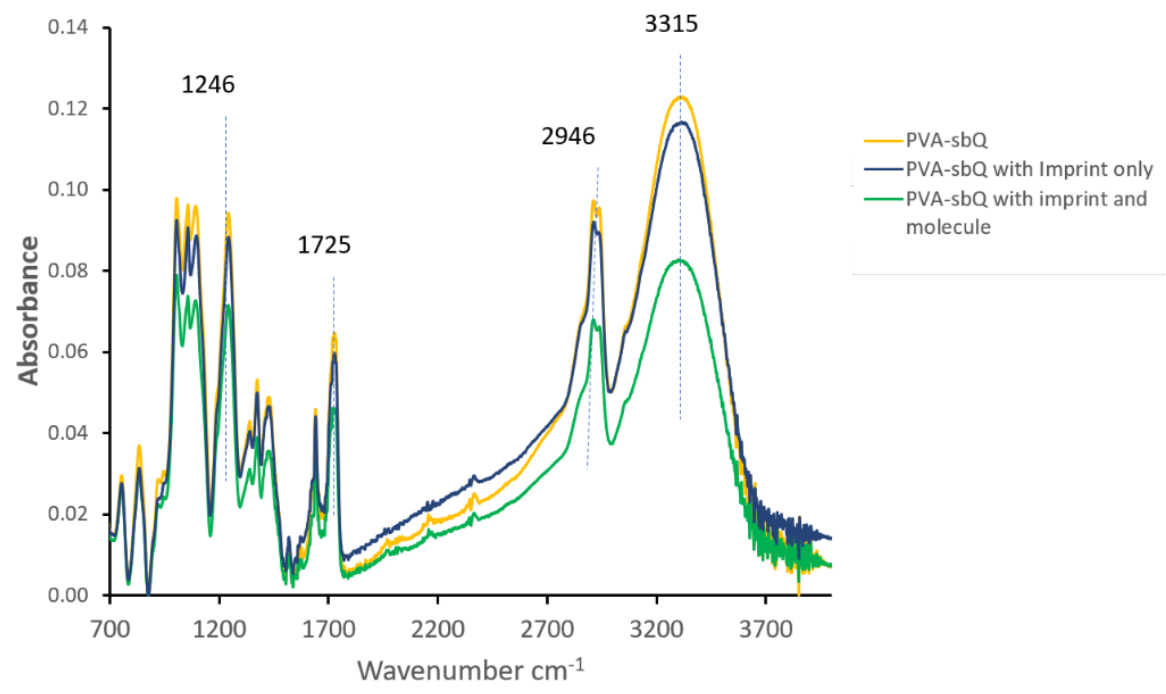

Figure 28 - FTIR Analysis

\subsection{Model Results}

The efficacy of the equivalent circuit model is analyzed in this section by comparing the impedance plots generated using the model parameters to the measured data. First the model results are analyzed when the measurement was ideal, and then the non-ideal measurements are explored to determine how much non-ideality is acceptable. The ideal measurement is a measurement where all three impedance regions are distinct, while for the non-ideal measurement the middle region has been shortened and is no longer clearly distinct. The model is then used to analyze the four IDEs outlined in Table 2 to select which IDE should be used moving forward.

\subsubsection{Model Matching}

From an ideal measurement two variations of the model were applied to the same data set, with a difference in the approach towards the double layer capacitance. The first model would attempt to improve the matching in the angle of the tail between the model and the data to obtain an accurate ideality factor, while the second model would prioritize 
the capacitance value of the double layer constant phase element. As shown by the Nyquist plot in Figure 29, the model 1 approach could match the angle of the tail, but this would lead to the double layer capacitance magnitude being underestimated. The model 2 approach would sacrifice the matching of the tail angle but would improve the impedance matching across the frequency sweep. The effects of the two methods of approach are further demonstrated in the impedance magnitude and phase plots. The method two approach gives an extremely high accuracy model of the impedance magnitude, with an average percentage error of $2.5 \%$ from a sample of 15 "ideal" measurements. The impedance phases error using method 1 matches at low frequency and high frequency; however, it cannot match the measured data phase in the middle frequency section. The method 2 approach has better accuracy in the middle frequency section but deviates further in the low frequency section. Due to the $a$ constant of the CPE affecting both magnitude and phase, the phase cannot be corrected without also affecting the magnitude.

The conclusion from this analysis, which was repeated for 15 different ideal

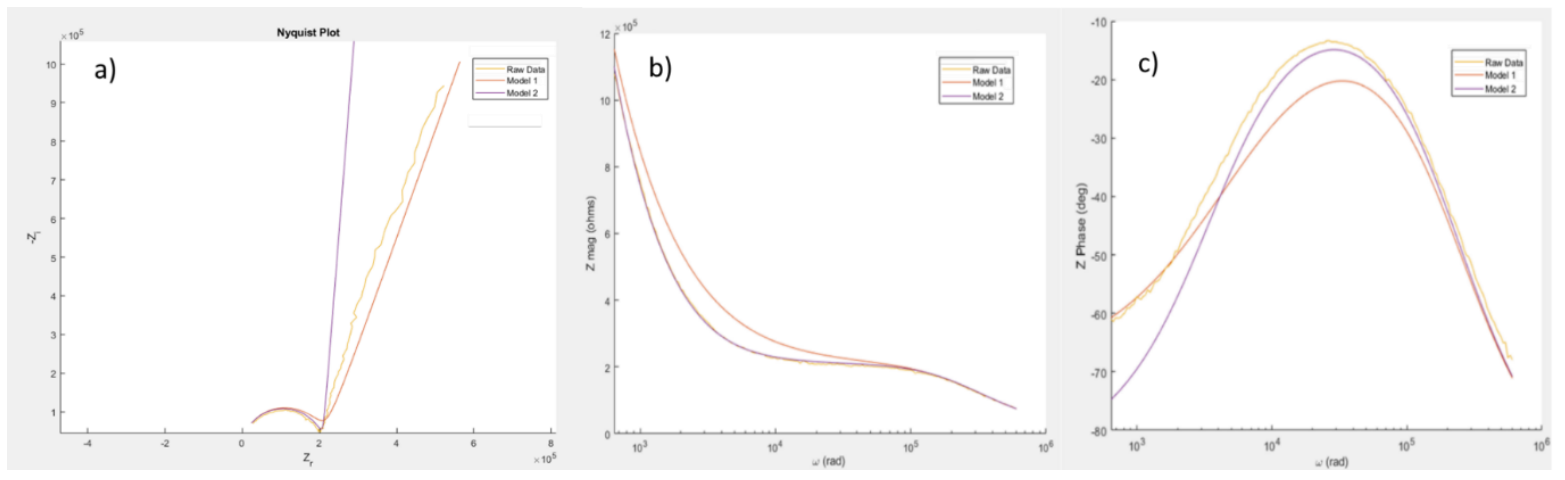

Figure 29 - Ideal Impedance Model vs Measurement with two approaches (a) Nyquist Plot (b) Impedance Magnitude Plot (c) Impedance Phase Plot

measurements with the same results, is that the model can achieve high matching to the measured data, but that the constant phase element for the double layer capacitor does not completely explain the low frequency characteristics of the sensor. It has been shown that this issue only occurs in the phase but not the magnitude, which may be due to external 
noise, error in the calibration at low frequency, or there are additional elements contributing to the low frequency phase of the impedance.

Both methods calculate the same resistances and geometry capacitance, and both cannot completely match the impedance phase, but the method 2 approach can match the impedance magnitude almost identically while the method 1 approach has a large error at low frequency. Therefore, the method 2 approach was utilized for all subsequent analyses.

Due the frequency shift mentioned in section 3.3 changing the corner frequencies, it is possible for the measured data to become non-ideal such as in Figure 23. The same model was used, which was able to demonstrate that it is still feasible to process these measurements, however it would have up to an additional $8 \%$ percentage error for the impedance magnitude. The results from one such non-ideal measurement is shown in Figure 30. The impedance magnitude is still able to retain relatively high precision, however as it attempts to create the three distinctive impedance regions where there is none, it results in an average percentage error below $10 \%$, as established from a sample of 10 measurements. The phase plot shows a much faster degradation in quality, depending on how good the data is. Although this is less than ideal, this model was only required during the initial test phase, as the optimized IDEs demonstrated very low frequency shift. 

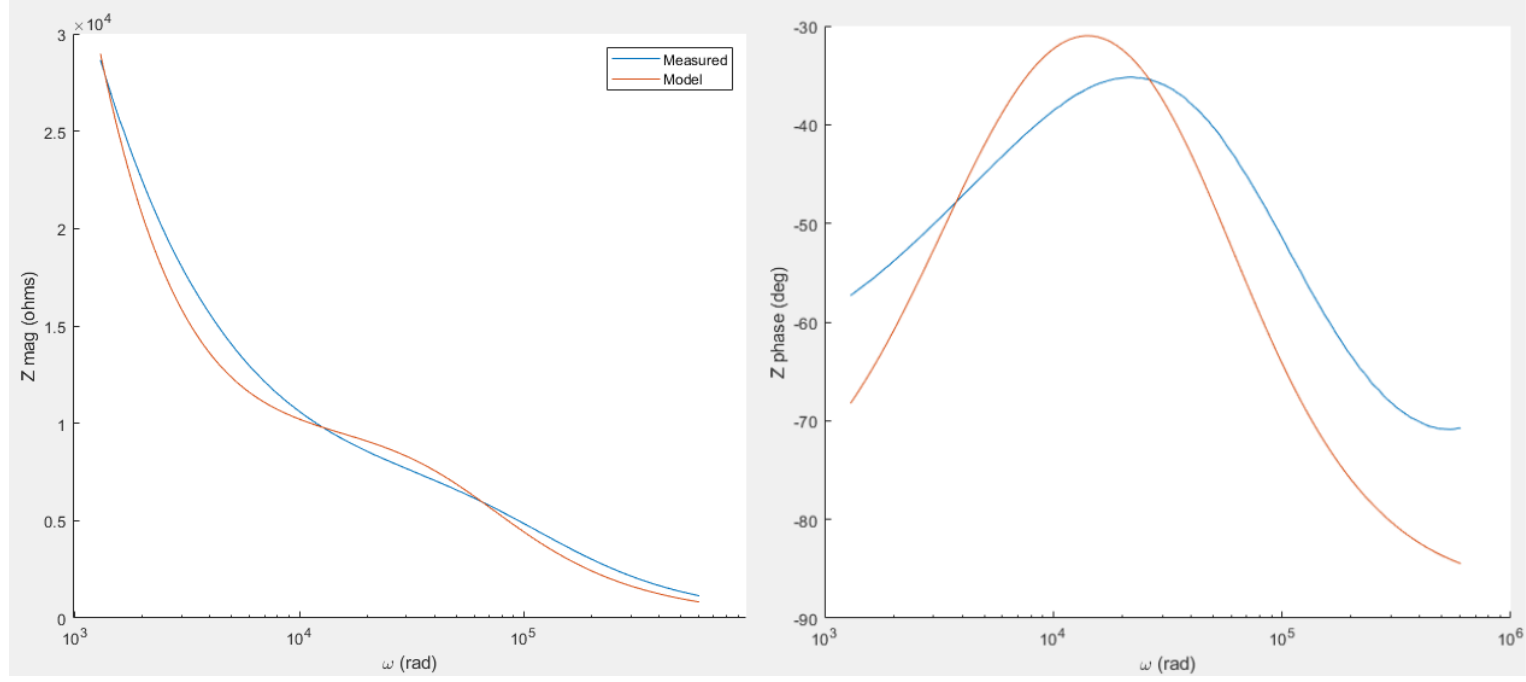

Figure 30 - Non ideal Impedance Model vs Measurement Magnitude and Phase

\subsubsection{IDE Comparison}

This section demonstrates the difference in the functionality between the four IDEs outline earlier. From a set of 4 measurements per sensors, the impedance models without samples are shown in Figure 31, followed by a comparison of their sensitivity at a concentration of $100 \mathrm{pg} / \mathrm{mL}$ in Figure 32. The extracted models match the theory; sensors A and B have the same electrode surface area, so their double layer capacitances are the same (same for sensors $\mathrm{C}$ and $\mathrm{D}$ ) while sensor $\mathrm{A}$ has a higher $\mathrm{C}_{\mathrm{G}}$ (as previously theorized) and a lower $\mathrm{R}_{\mathrm{S}}$ due to having a smaller cell constant. From equation 3.12, the change in geometry capacitance is proportional to the change in dielectric and inversely proportional to the cell constant, so the lower cell constant of sensor A means that it has a larger change in capacitance compared to the rest, which will make low concentrations of samples easier to detect. Sensors A and B have larger sensitivities due to the larger electrode area, while sensor A has a smaller standard deviation than sensor B due to having more consistent modeling with the larger capacitance changes. 


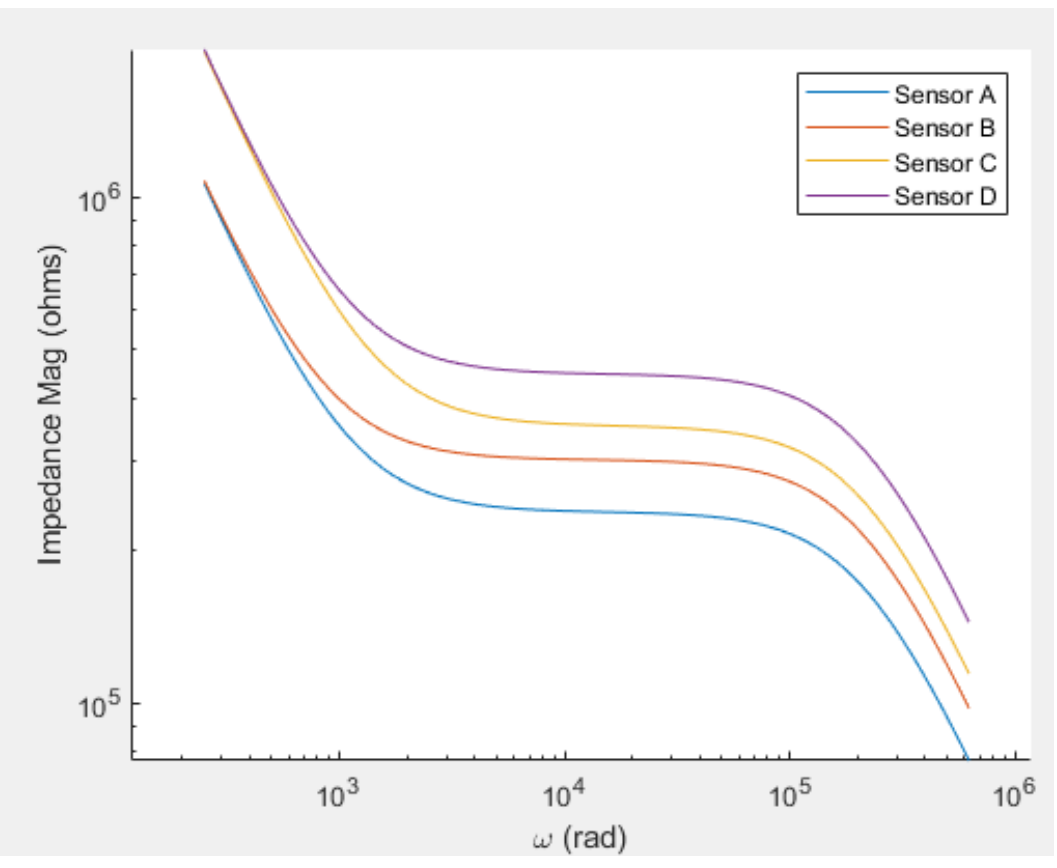

Figure 31 - IDE models ( $n=4$ per sensor)

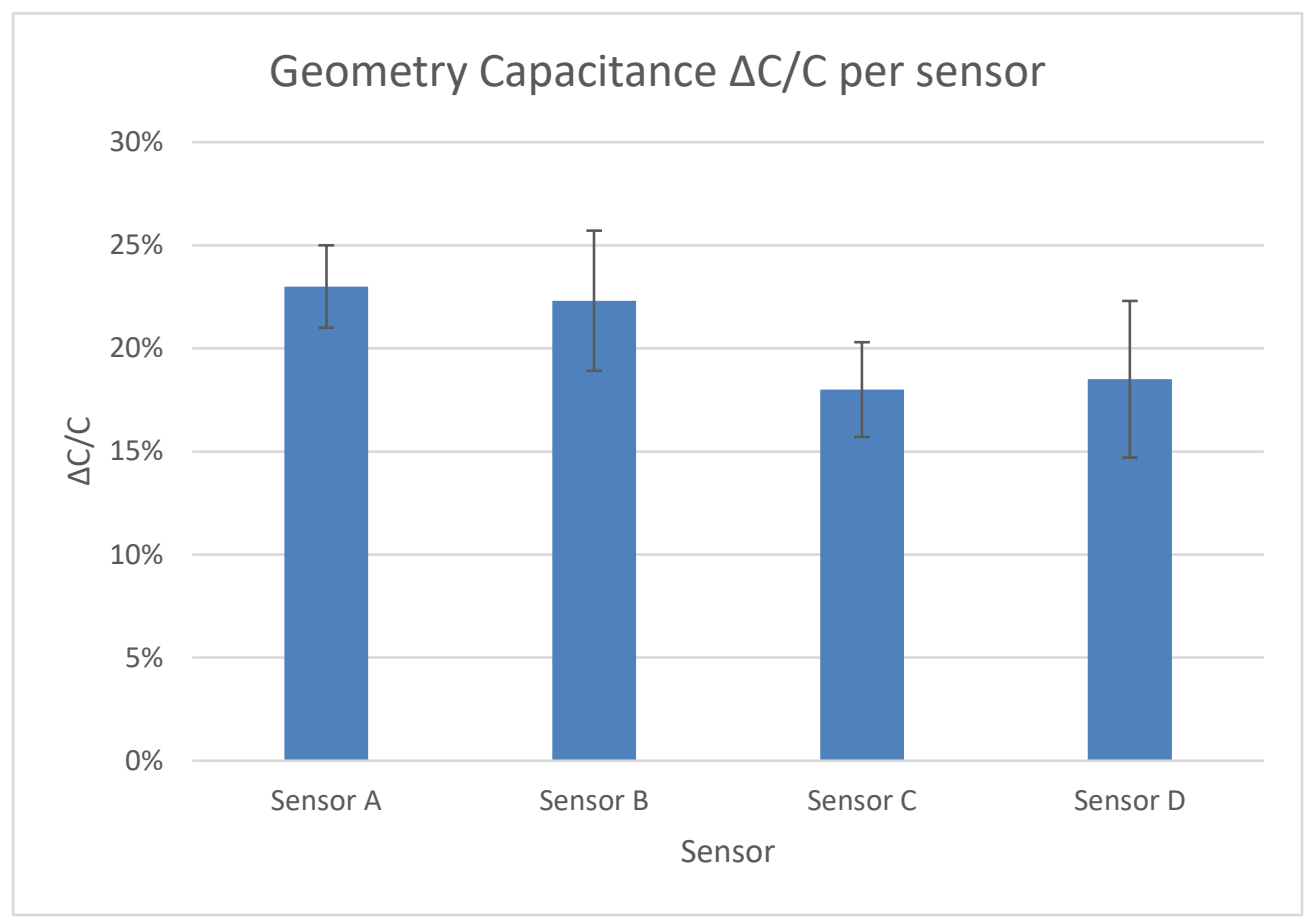

Figure 32 - Comparison of Sensor Sensitivity at $100 \mathrm{pg} / \mathrm{mL}$ ( $\mathrm{n}=6$ per sensor) 


\subsection{Sensor Performance in Aqueous and Aerosolized Sample Testing}

\subsubsection{Aqueous Sample Results}

The initial experiments were performed with the samples in an aqueous solution at high concentrations as a proof of concept using concentrations higher than in exhaled breath. The MIPs coatings were built on un-optimized IDEs and required larger $1 \mathrm{MHz}$ frequency sweeps. After using initial measurements to characterize the model, the characteristics of the sensor were determined using concentrations from $1 \times 10^{4} \mathrm{pg} / \mathrm{mL}$ to $1 \times 10^{9} \mathrm{pg} / \mathrm{mL}$. First, the time response is measured to determine when the sensor readout will stabilize. Figure 33 demonstrates a standard measurement of an unoptimized sensor parameters after a sample of 8-isoprostane has been dropped. From Figure 33, the geometry capacitance and the double layer capacitance are analyzed over a course of 4 minutes, with the $\mathrm{C}_{\mathrm{G}}$ parameter showing a sharp increase in capacitance after the sample is applied, followed by the measurement stabling at around 30 seconds, followed by a constant drift. It is believed that this drift is due to water being absorbed into the hydrogel, as the aerosolized solution tests showed much less drift with time. The large increase in capacitance immediately after the sample was applied is believed to be due to a large, localized concentration of the 8-isoprsotane, before it has had time to spread out over the sensing surface. The double layer capacitance appears to stabilize after 30 seconds and stays roughly constant afterwards. Due to the previously mentioned issues with the model, the double layer capacitance parameter extraction is less accurate, and the value is less constant. However, as it is not the transconductance parameter, this inaccuracy is considered to be acceptable as the increase of double layer capacitance due to surface 
interactions is still detectable. The rest of the measurements in this section were all taken 60 seconds after the sample was exposed to ensure a fair comparison between tests.

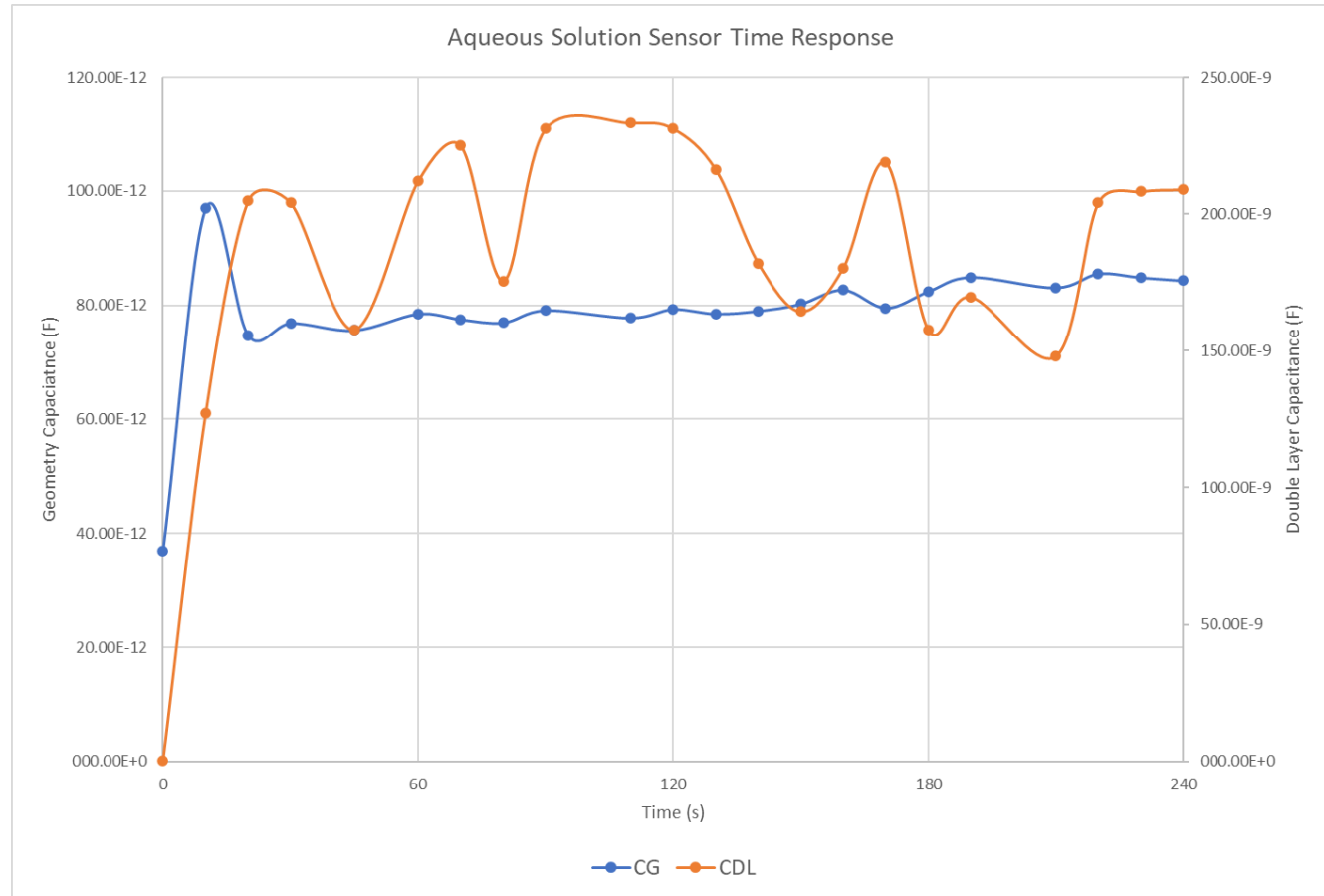

Figure 33 - Sensor Response Time for Aqueous Samples

Knowing the response time of the sensor, the sensitivity of the sensors prepared using stamps 1 and 2 were then compared in Figure 34 to detect the sensitivity and saturation limits for both. As expected, the sensitivity of the sensors developed with stamp 1 have almost twice the sensitivity and a higher saturation limit as it has double the concentration of 8 -isoprostane on the stamp. The sensor 1 stamp saturates around 0.1 $\mathrm{mg} / \mathrm{mL}$ of 8 -iso with a volume of $5 \mu \mathrm{L}$. This concentration is an order of magnitude below the concentration on stamp 1 , which was $1 \mathrm{mg} / \mathrm{mL}$. The saturation limit pf the stamp 2 sensor appears to occur between $0.1 \mathrm{mg} / \mathrm{mL}$ and $0.01 \mathrm{mg} / \mathrm{mL}$, which is consistent with the results of stamp 1 since the concentration of stamp 2 was $0.5 \mathrm{mg} / \mathrm{mL}$. Since these concentrations are much larger than the physiologically relevant range, both stamps are 
usable for the sensor, however since the stamp 1 sensors have a higher sensitivity, all further measurements were taken with sensors using stamp 1.

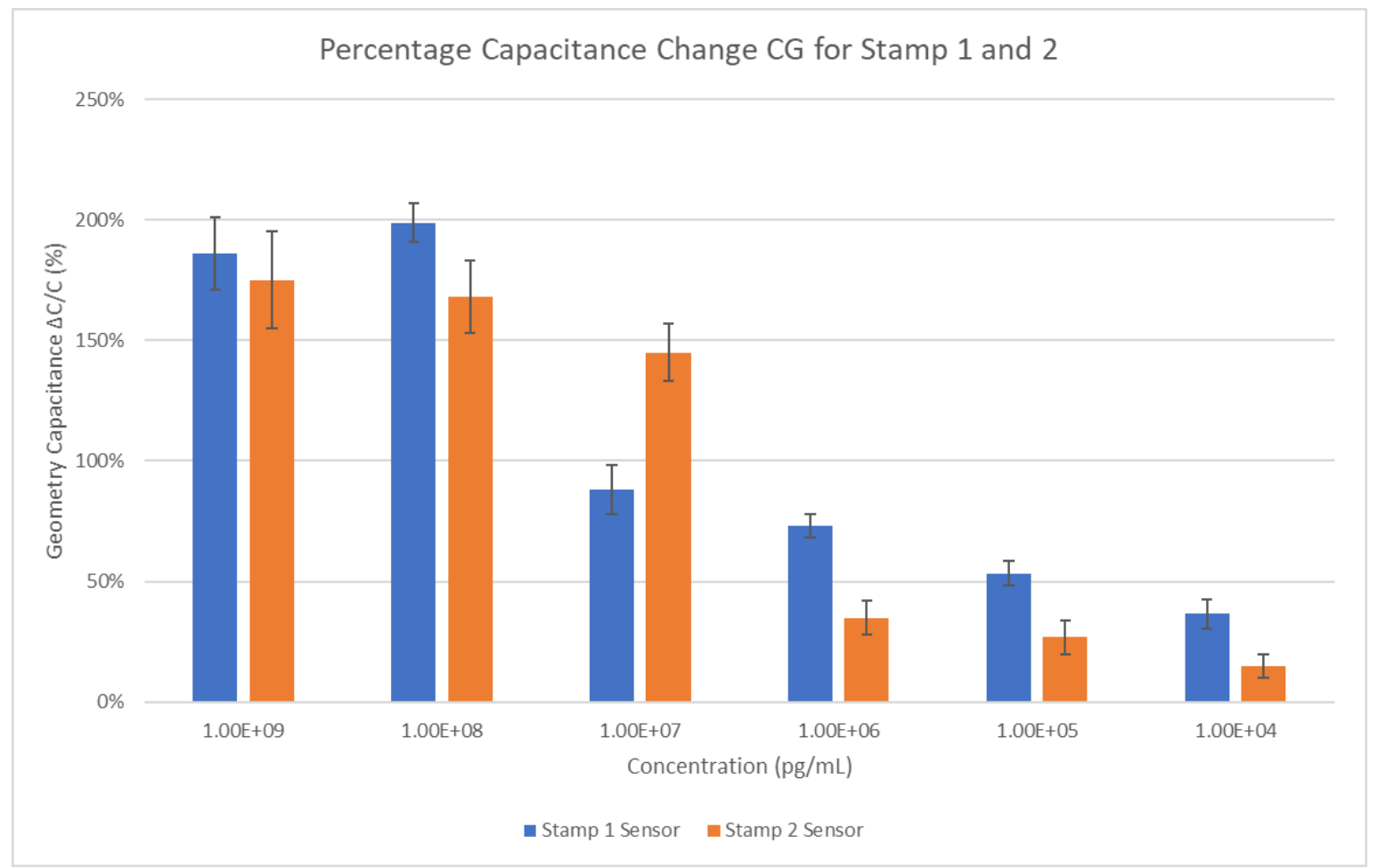

Figure 34 - Stamp 1 and 2 Sensor Comparison ( $n=7$ per sensor)

\subsubsection{Cross-sensitivity Validation of the MIP-EIS Sensor}

The following step was to determine the cross-sensitivity of the sensors towards the other similar molecules: cortisol, cortisone, and progesterone. The same amount of all the samples were applied to the sensor, and then the impedance response was modeled. To avoid cluttering the graph in Figure 35 the error bars for the other molecules are not shown, however the standard deviation for the majority of the measurements is within $10 \%$ of the mean. As shown in Figure 35, the sensor demonstrates high selectivity against those molecules except for cortisol. The nature of the cross-sensitivity towards cortisol is still unknown, both cortisol and cortisone have -OH functional groups in the same locations so 
it would be expected that they have similar cross-sensitivities, however no such crosssensitivity occurs with cortisol. Cortisol has a hydroxide group that is not present in cortisone, which may be the cause of the cross-sensitivity. This high cross-sensitivity only occurs in the high concentration regions, and in the aerosolized concentration the selectivity of the sensor is shown to be improved.

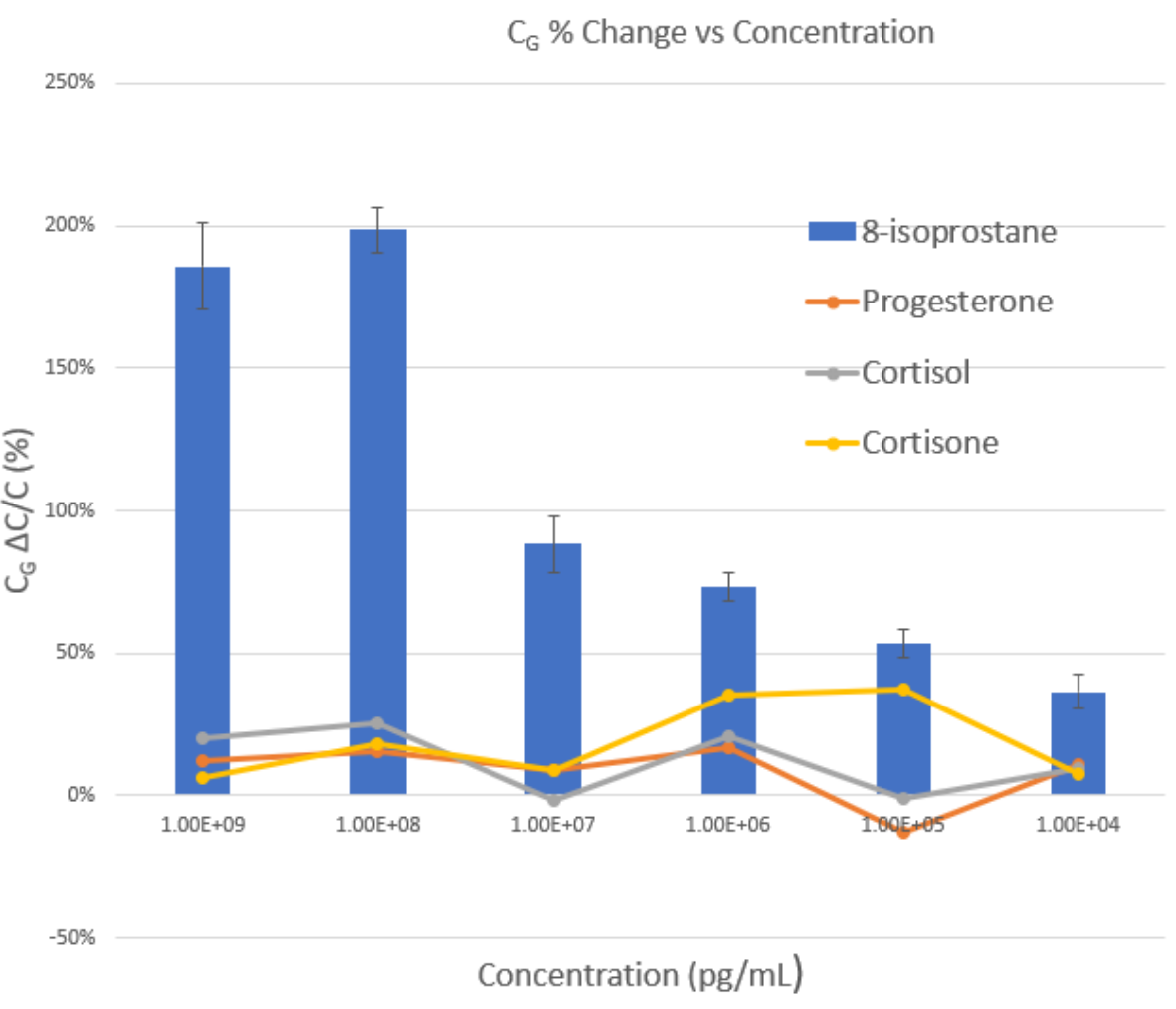

Figure 35 - Aqueous Solution Selectivity ( $n=7$ per sensor)

\subsubsection{Aerosolized Sample Testing}

The aerosolization microchannel was used to create spiked aerosol samples with 8isoprostane levels varied between 100 and $1 \mathrm{pg} / \mathrm{mL}$, mimicking the concentration range found in the exhaled breath condensate. A lower frequency sweep was used to lower the measurement time and to make it feasible for chip-based impedance measurement which is limited to $100 \mathrm{kHz}$. The response time was found to have increased to around $60 \mathrm{~s}$ as 
shown in Figure 36, which represents an average sample measurement with a sample of $100 \mathrm{pg} / \mathrm{mL}$ applied. A sharp increase in the geometry capacitance occurs again as soon as the sample is applied, but the sensor response becomes much more consistent, likely due the samples being better distributed along the surface due to the aerosolization method. Due the optimized sensors, the double layer capacitance measurement is much more consistent than before. The measurements in Figure 37, which demonstrate the comparative response towards the different samples and concentrations, were all taken 60 seconds after the sample was applied. With the low concentrations, it is important to wait for the measurement to stabilize to ensure a proper reading. The sensor response again shows some cross-sensitivity, especially towards cortisone. At $100 \mathrm{pg} / \mathrm{mL}$, there is almost a $50 \%$ sensitivity towards cortisone, but by analyzing double layer capacitance as well such as in Figure 38, the selected model can reliably distinguish between the two assuming the sample volume is known. 


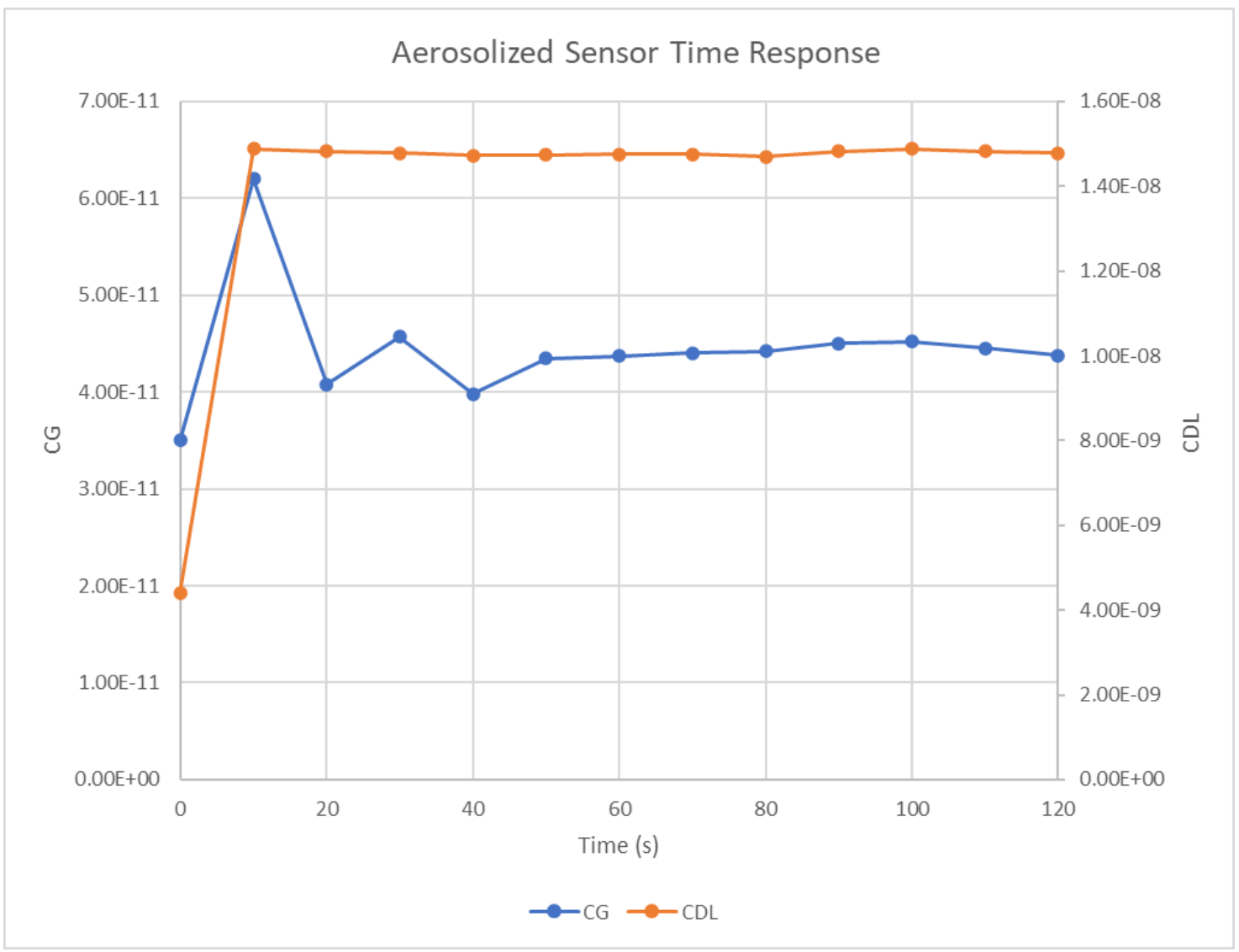

Figure 36 - Aerosolizes Sensor Time response

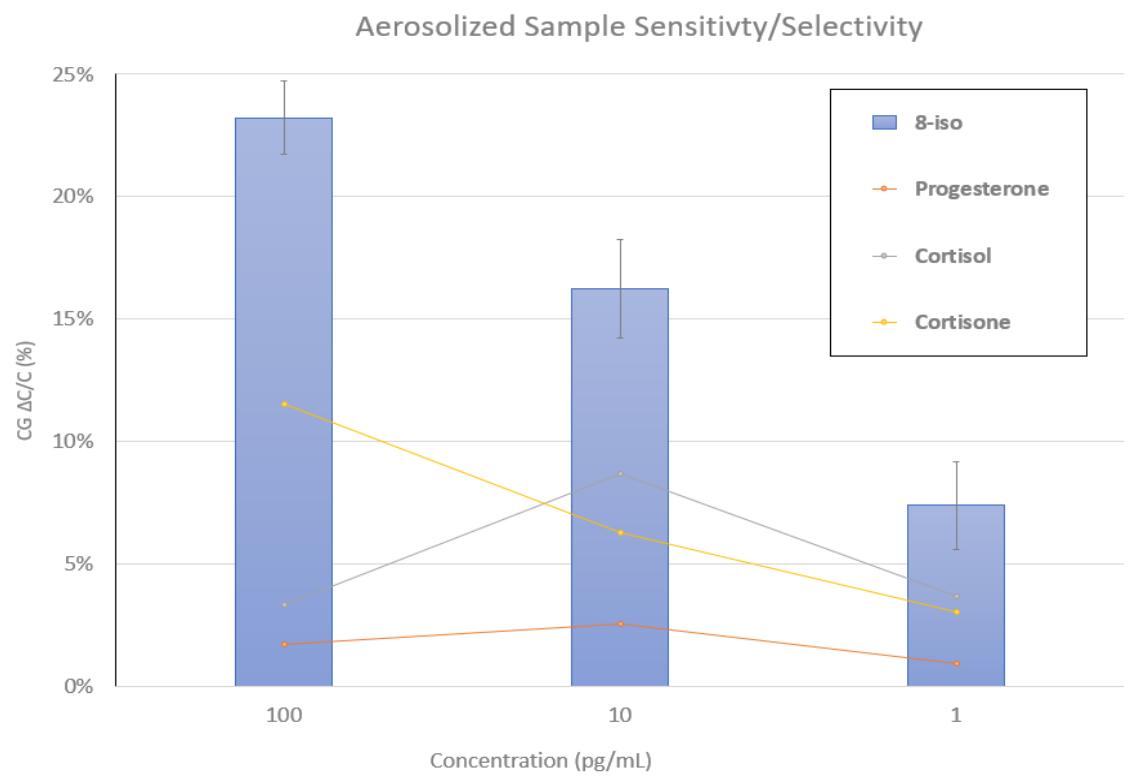

Figure 37 - Aerosolized Sensor CG Response ( $n=6$ per sensor) 
The larger increase in the double layer capacitance shown in Figure 38 is used to separate a low concentration of cortisone versus a low concentration of 8 -isoprotane. The limit of detection of this sensor has been shown to be down to $1 \mathrm{pg} / \mathrm{mL}$ and is able to detect concentrations up to $0.1 \mathrm{mg} / \mathrm{mL}$. The integration of the sensor with the PCB is in its final stages, with the board completed and the board prepared to test once the sensor is bonded onto the PCB.

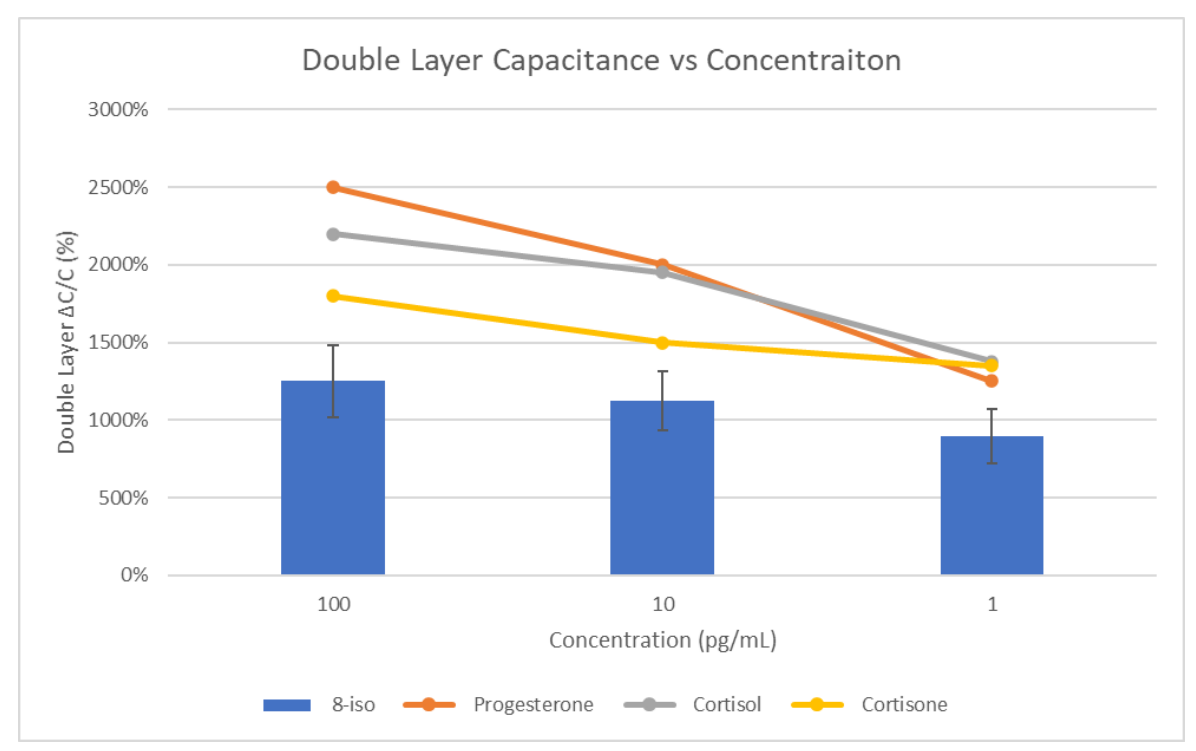

Figure 38 - Aerosolized Sensor CDL Response ( $n=6$ per sensor)

\subsection{Packing and Integration}

Once the sensor proven to work, a circuit board was designed to incorporate the sensor, the impedance measurement, the microfluidic channels, and the model analysis. The design was also expanded to include a completely multiplexed system, able to analyze up to four EIS sensors and 11 organic electrolyte-gated FETs (OEGFET). The addition of the OEGFET sensor was a collaboration to demonstrate the integration of multiple low-cost small footprint technologies [83]. With the sensors and readout systems integrated into one package, the signal noise can be reduced, and the readout time greatly sped up. The impedance and OEGFET analysis can all be done locally on the board and 
exports the calculated ECM model parameters and OEGFET parameters directly to a PC. Both sensors utilize a custom package, with the substrate directly bonded to the PCB, the connections wire bonded to bonding pads on the $\mathrm{PCB}$, and microfluidic channels built directly on the sensors. Figure 39 shows the developed board with a wire bonded MIPsEIS chip and the aerosolization chamber.

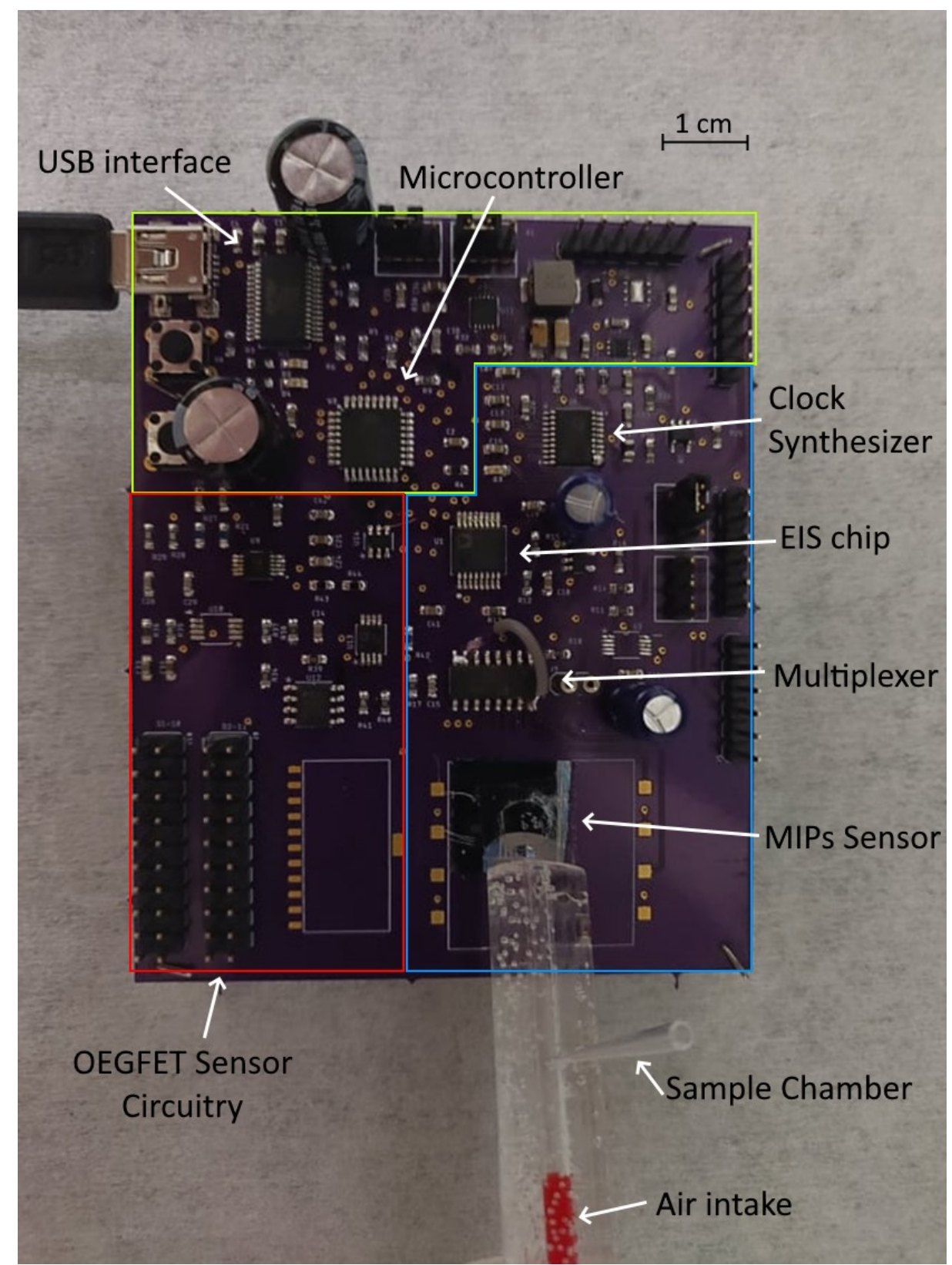

Figure 39 - Integrated PCB-Sensor Assembly 
The board was designed to require minimum current, requiring approximately 10 $\mathrm{mA}$ for the control and power section of the board, and a total of $20 \mathrm{~mA}$ to run all the sensors. The device is USB powered, allowing it to be controlled via any computer or laptop, making it very portable. Figure 40 shows a sample measurement taken with the integrated board, demonstrating that the three impedance regions are obtainable with this method and the model can be easily applied.

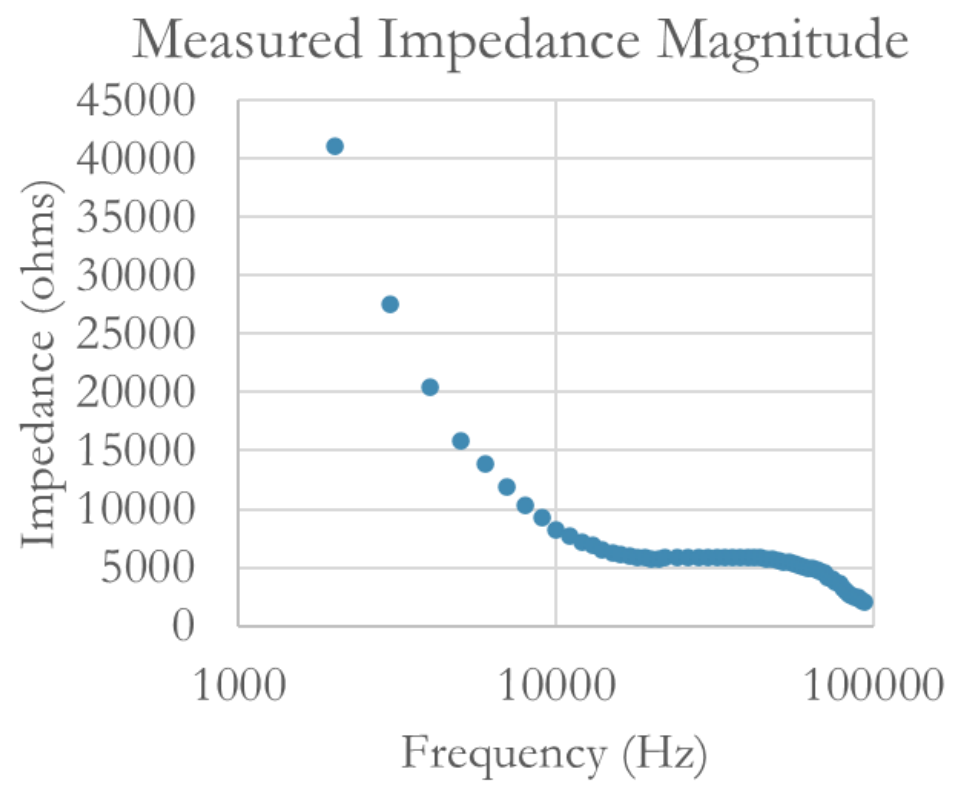

Figure 40 - Sample EIS measurement from integrated board

The ATMEGA328 8-bit microcontroller [84] was selected as the board controller as it commonly found in open-source Arduino based projects. The bootloader can be flashed using the SPI interface and then the board can be reprogrammed utilizing the USB interface. In order to interface the ATMEGA328 to a computer USB, the FT232RL [85] USB to serial UART interface chip is used. The board can be powered from either the USB $5 \mathrm{~V}$ supply or an external $5 \mathrm{~V}$ supply, which is selecting using a jumper. The various components on the board require a clean ripple-free $5 \mathrm{~V}$ signal, and the $5 \mathrm{~V}$ signal from the 
USB interface is too noisy to use directly. Furthermore, the OEGFET test system requires a $0-6 \mathrm{~V}$ sweep with only a $5 \mathrm{~V}$ input. The voltage input is therefore connected to the ADP1614 step-up DC-to-DC switching converter to convert the $5 \mathrm{~V}$ input to a $6 \mathrm{~V}$ signal with a settling time of $500 \mathrm{~ms}$ [86]. The ADP1614 has excellent noise filtering and with carefully selected external components produces a $6 \mathrm{~V}$ signal will less than $10 \mathrm{mV}$ ripple from a noisy input. The $6 \mathrm{~V}$ signal is then passed through the ADP7118 linear regulator to convert the voltage back to $5 \mathrm{~V}$ to be used to power the circuitry on the board [87]. Digital temperature and humidity sensors are located on the board to monitor ambient conditions and detect voltage drift.

The AD5933 network analyzer was selected to perform the impedance analysis and can return the real and imaginary components of an unknown impedance [88]. The AD5933 applies a $1.98 \mathrm{~V}$ peak-to-peak signal with a $1.48 \mathrm{~V}$ offset and has a 2 gain stages on the return signal. This signal can measure resistance values from $10 \mathrm{M} \Omega$ down to $1 \mathrm{k} \Omega$, however some sensors may require have impedances below $1 \mathrm{k} \Omega$. In order to decrease the impedance range that can be measured, the applied signal can be attenuated by changing two resistors as the signal is passed through an amplifier. Since the presented sensor has a very high coating resistance, no attenuation, however for other low impedance applications setting these resistors to $4 \mathrm{k}$ and $20 \mathrm{k}$ will attenuate the signal to $400 \mathrm{mV}$ peak-to-peak signal. The offset is set to $2.5 \mathrm{~V}$ in order to have it at mid-voltage; the offset is acceptable as it is common-mode and will not create a charge on the sensor surface. With 4 possible sensors, as well as 3 known passive components and one external component for calibration, the 74HC4051D is used as an 8:1 analog bi-directional multiplexer/demultiplexer (MUX/DEMUX) is used as a DEMUX to select to what device the excitation signal is sent 
to [89]. The known value passives are used to perform calibration, or an external device can be connected of any value for the calibration. The first gain stage of the return signal is set by a resistor, which can either be one of two set values, or can be set from $320 \Omega$ to $100 \mathrm{k} \Omega$ using the AD5248 digital potentiometer [90]. The second gain stage is set internally on the AD5933 by modifying the gain registers. The measurement across the $100 \mathrm{kHz}$ frequency spectrum needs to be done in stages, as different clock frequencies are required for different frequency ranges. The AD9834 direct digital synthesis (DDS) chip is used as variable clock generator, able to generate clocks down to $1 \mathrm{kHz}$ for low frequency measurements and up to $4 \mathrm{MHz}$ for high frequency measurements [91]. The four impedance sensors can be configured a variety of ways: the optimal configuration for the MIPs exhaled breath sensor is to have one imprinted sensor, one non-imprinted sensor, a thin film capacitive humidity sensor, and a reference sensor for the humidity sensor. The combination of the imprinted and non-imprinted sensor provides a fully differential sensor to improve sensor response, while the differential humidity sensor improves sample sensing and is used to determine the amount of sample on the sensor.

The OEGFET measurement requires the extraction of the IV characteristics curves of the PMOS devices. The devices required a $6 \mathrm{~V}$ sweep and have a maximum current of $500 \mu \mathrm{A}$. To sweep VGS and VDS, the source is set to a constant $6 \mathrm{~V}$ and VG and VD are controlled via a digital to analog converter (DAC). The AD5663 DAC can provide a 0 to $5 \mathrm{~V}$ output [92], which is then amplified by a factor of 1.2 to provide a 0 to $6 \mathrm{~V}$ output range. Between the PMOS drain and the DAC drain output is a 20-ohm current sensing resistor. The current sensing resistor is monitored by the AD8210 current shunt monitor, which outputs a voltage proportional to sensed current [93]. This voltage is then amplified 
and passed through the AD7680 [94] analog to digital converter, which is then sent to the microcontroller to analyze. In most cases, the current sensing resistor should be in the range of $0.5 \Omega$ to $1 \Omega$, however due to the very small current range it was required to increased to $20 \Omega$ to ensure a large enough voltage swing. Due the relatively large current sensing resistor, the voltage on the drain will be slightly different than the drain voltage generated by the DAC. However, since the current will be known the drain voltage on the sensor is simply

$$
V_{D, P M O S}=V_{D, D A C}-I_{S E N S E} R_{S E N S E}
$$

The OEGFET sensor array consists of 11 series PMOS devices with a common gate (shown in Figure 45 in the appendix). In order to select which PMOS in the array is to be measured, the source and drain are selected using low resistance jumpers. Due to the low current of the OEGFETs and the $6 \mathrm{~V}$ voltage range, digital analog multiplexers could not be used; to ensure a multiplexer does not interfere with the current measurements the on resistance of the multiplexer must be significantly smaller than the current sensing resistor. While there are analog multiplexers with on resistances well below $1 \mathrm{ohm}$, they are designed for low voltage use, and will not work with the required 6-volt range. The analog multiplexers designed to handle the required voltage range have on resistances with the range of, or larger than, the current sensing resistor, and therefore cannot be used. The manual jumpers are there for use, which have negligible resistance. 


\section{Chapter 6: Conclusion}

This report has explored the use of MIPs technology for exhaled breath condensate by simulating the condensate with spiked aerosolized solutions. After the materials were selected by finding a high dielectric, photo-cross linkable, non-conductive polymer that can form hydrogen bonds with the target, a low-cost rapid-production method was implemented to produce the sensors. Using a soft-lithography technique, a highly reusable PDMS stamp with the template was developed, which was used to create a $250 \mathrm{~nm}$ thick molecularly imprinted PVA-SbQ film. A critical oxidate stress biomarker, 8-isoprostane, was used to prove importance of the sensor as it has been demonstrated to be useful in the detection on interstitial lung diseases. The proven sensor shows the "generic" MIPs approach is viable and can be expanded towards other targets. Other molecules with -OH functional groups can theoretically be used with PVA-SbQ to develop a MIPs-EIS sensor targeted to those molecules.

The importance of the IDE structure and the coating properties towards the impedance of the sensor were analyzed using numerical methods, then applied to optimize the IDE dimensions. The various IDEs were then tested to confirm they conformed with the theory. An analysis of the stamp and IDE surfaces with different imaging techniques was performed to correlate the sensor surface properties to they sensor performance. It was shown through FTIR that an increase in hydrogen bonds is present when the sample is present, indicating that the molecules haves formed a weak physical connection the polymer surface. Sensors with various IDEs, stamp template densities, and sample concentrations were studied using an equivalent circuit model to fully understand how the 
sensor works and how it depends on the various parameters. A custom algorithm was implemented to analyze the impedance data and translate it to the 4-element circuit model. The effectiveness of the model was also analysed, showing that the impedance magnitude can be matched with extremely high precision with some discrepancy between the model and the data phase due to the implementation of the constant phase element,

The results, summarized in Figure 41, demonstrate the wide sensing range of the sensor and breaks down the two stages of testing. The aerosolized testing shows a limit of detection down to $1 \mathrm{pg} / \mathrm{mL}$ and a logarithmic relationship between the change in capacitance and the 8-isoprostane concentration. The complete model data was used to determine the relationship between the change in impedance and the concentration. Compared to an antibody based electroimpedance spectroscopy 8-isoprostane sensor, this work demonstrated an improved limit of detection of $1 \mathrm{pg} / \mathrm{mL}$ compared to the reported 12 $\mathrm{pg} / \mathrm{mL}$ [95]. The antibody-based sensors have also demonstrated improved selectivity over our approach due to the generic material approach. A custom PCB was then developed to fully integrate the sensor alongside a PMOS OEGFET.

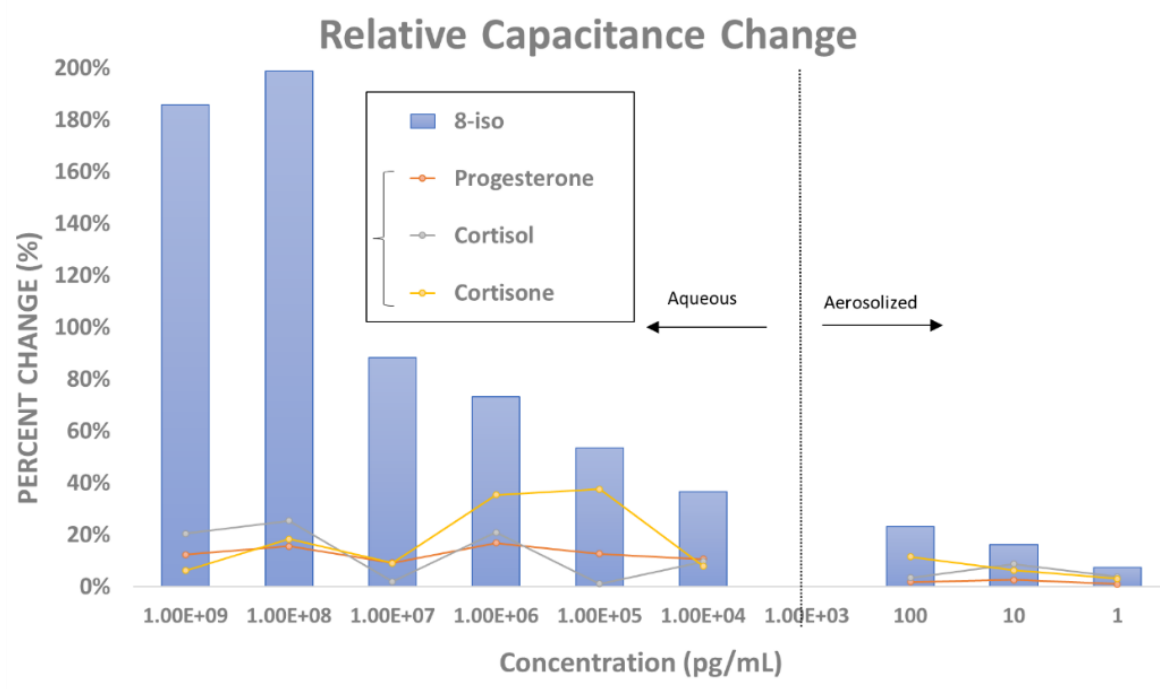

Figure 41 - Summary of Results 


\section{Chapter 7: Future Work}

This work has shown a low-cost, non-invasive, point-of-care sensor that can simplify the testing of 8 -isoprostance in exhaled breath. With proper integration, the molecularly imprinted polymer-based sensor can achieve a small footprint sensor with improved sensitivity/selectivity, longer shelf life, and automated modeling. The device currently uses a passivated silicon substrate, and it can be synthesized in-house, at low cost. PVA is known for having excellent mechanical properties, and by using the equations in the optimization section, the device could be remade to utilize a flexible substrate. The flexible substrate would be ideal as it be made cheaply and with more environmentally friendly materials, while also expanding the use of the sensor as it no longer needs to remain on a flat surface. The understanding of the sensor can also used to reduce the sensing area so further reduce the footprint of the sensor.

The sample collection method needs to be implemented to have a complete exhaled breath condensate sensor, which could be implemented in a similar fashion as a breathalyser. The addition of soft microfluidics would allow for the sample/reagents to be moved from collection reservoirs to the sensor, then removed and the alcoholic wash buffer could be added, and finally removed from the sensor. The addition of a microfluidic channel would also improve the control of the amount of sample on the sensor.

The application of the sensor should be further analyzed for multi-analyte samples to improve the understanding of the sensor in those applications. To improve the sensing ability of the sensor, a non-imprinted sensor should be used alongside the imprinted sensor. Since exhaled breath condensate contains a wide variety of different molecules which would all be present of the sensor surface on the same time, the differential measurement 
between the imprinted and non-imprinted will be modeled to ensure the sensor can still detect the target.

The model can be updated to function with the differential multi-analyte setup. Although the sensor is minimally affected by temperature variation, the addition of the temperature sensor can be used to minimize sensor drift during operation across a broad range of temperatures. The integration of humidity sensors next to the samples can be used to determine the volume of aerosolized sample present to further improve the signal analysis. The integrated packaging should perform the data analysis locally and output the final detected concentration to a pc or a mobile device. The data can be safely stored and used by health care professionals for continuous health monitoring.

Finally the generic MIPs production method can be used to study its feasibility towards other targets, such as Prostaglandin $\mathrm{F}_{2 \alpha}\left(\mathrm{PGF}_{2 \alpha}\right)$ which has been shown to be an another biomarker for oxidative stress, and the ratio between 8 -iso and $\mathrm{PGF}_{2 \alpha}$ is believed to provide a more precise measurement for oxidative stress [96]. 


\section{Appendices}

\section{Appendix A : Impedance Equation Proof}

First, the coating resistance and the double layer capacitance are in series and have impedance $Z_{1}$.

$$
Z_{1}=R_{S}+\frac{1}{(j \omega)^{\alpha} C_{D L}}
$$

$Z_{2}$ is the parallel combination of $Z_{1}$ and the geometry capacitance impedance. This equation is then expanded.

$$
\begin{gathered}
Z_{2}=\left(j \omega C_{G}+\frac{1}{R_{S}+\frac{1}{(j \omega)^{\alpha} C_{D L}}}\right)^{-1} \\
Z_{2}=\left(j \omega C_{G}+\frac{(j \omega)^{\alpha} C_{D L}}{(j \omega)^{\alpha} C_{D L} R_{S}+1}\right)^{-1}=\frac{(j \omega)^{\alpha} C_{D L} R_{S}+1}{(j \omega)^{\alpha} C_{D L}+j \omega C_{G}+(j \omega)^{\alpha+1} R_{S} C_{G} C_{D L}}
\end{gathered}
$$

The total impedance is then $Z_{2}$ plus $R_{C}$. First $\alpha$ is set to 1 , then the complex part of the impedance is multiplied by its conjugate. Then similar terms are grouped and separated into the real and imaginary components.

$$
\begin{gathered}
Z=R_{C}+Z_{2} \\
Z=R_{C}+\frac{j \omega C_{D L} R_{S}+1}{j \omega\left(C_{G}+C_{D L}\right)-\omega^{2} C_{G} C_{D L}} \quad \alpha=1 \\
Z=R_{C}+\frac{j \omega C_{D L} R_{S}+1}{-\omega^{2} C_{G} C_{D L}+j \omega\left(C_{G}+C_{D L}\right)} \frac{-\omega^{2} C_{G} C_{D L}-j \omega\left(C_{G}+C_{D L}\right)}{-\omega^{2} C_{G} C_{D L}-j \omega\left(C_{G}+C_{D L}\right)}
\end{gathered}
$$




$$
\begin{gathered}
Z=R_{C}+\frac{\left[\omega^{2} R_{S} C_{D L}\left(C_{D L}+C_{G}\right)-\omega^{2} R_{S} C_{D L} C_{G}\right]-j\left[\omega\left(C_{D L}+C_{G}\right)+\omega^{3} R_{S}^{2} C_{D L}^{2} C_{G}\right]}{\omega^{2}\left(\omega R_{S} C_{G} C_{D L}\right)^{2}+\omega^{2}\left(C_{G}+C_{D L}\right)^{2}} \\
Z=R_{C}+\frac{R_{S} C_{D L}^{2}}{\left(C_{G}+C_{D L}\right)^{2}+\left(\omega R_{S} C_{G} C_{D L}\right)^{2}}-j \frac{C_{G}+C_{D L}+C_{G}\left(\omega R_{S} C_{D L}\right)^{2}}{\omega\left[\left(C_{G}+C_{D L}\right)^{2}+\left(\omega R_{S} C_{G} C_{D L}\right)^{2}\right]}
\end{gathered}
$$

\section{Appendix B : Additional Board Design}

Figure 42 and Figure 43show the analog front end for the AD5933 network analyzer chip. The design philosophy while creating the design was to create a system that would work for a range wide range of impedances, not just the sensor developed for this project. First, in the figure below the gain is set by R16/R13, which can be used to attenuate the output signal of the AD5933 for low impedance measurements. The signal output has a 2.3 $\mathrm{V}$ DC voltage, and the ac signal is applied on top, so the common mode voltage is $2.3 \mathrm{~V}$ plus the ac signal amplitude. For a gain of 1 , the common mode voltage is $3.3 \mathrm{~V}$, while a gain of 0.2 (minimum recommended gain) will set the common mode to $2.5 \mathrm{~V}$. Any 0605 footprint device can be soldered onto the board for the on-board calibration, shown on the schematic with a $1 \mathrm{k} \Omega$ resistor (replaced with a $100 \mathrm{k} \Omega$ resistor on the board), $10 \mathrm{k} \Omega$ resistor, and $1 \mathrm{nF}$ capacitor. The last DEMUX output is connected to an external connecter to be used as an external calibration. The other use of the amplifier is to ensure a constant low output impedance. The AD5933 has an internal $100 \mathrm{kHz}$ low pass filter on the input, so no additional signal filtering was required. The clock generator in Figure 44 has two outputs, the non-inverted and inverted outputs. R22 is used to set the output current to 3 $\mathrm{mA}$, and the output resistor is set to $200 \mathrm{ohms}$ to set the output voltage swing to $600 \mathrm{mV}$. The output voltage is AC bypassed and the DC offset is set to mid-rail. The amplifier is 
used to set the output impedance to $50 \mathrm{ohms}$, and a $4.5 \mathrm{MHz}$ low pass filter is used to reduce jitter on the output. This signal is then fed back to a comparator in the chip, which converts the sinusoidal waveform to a digital clock of the same frequency.
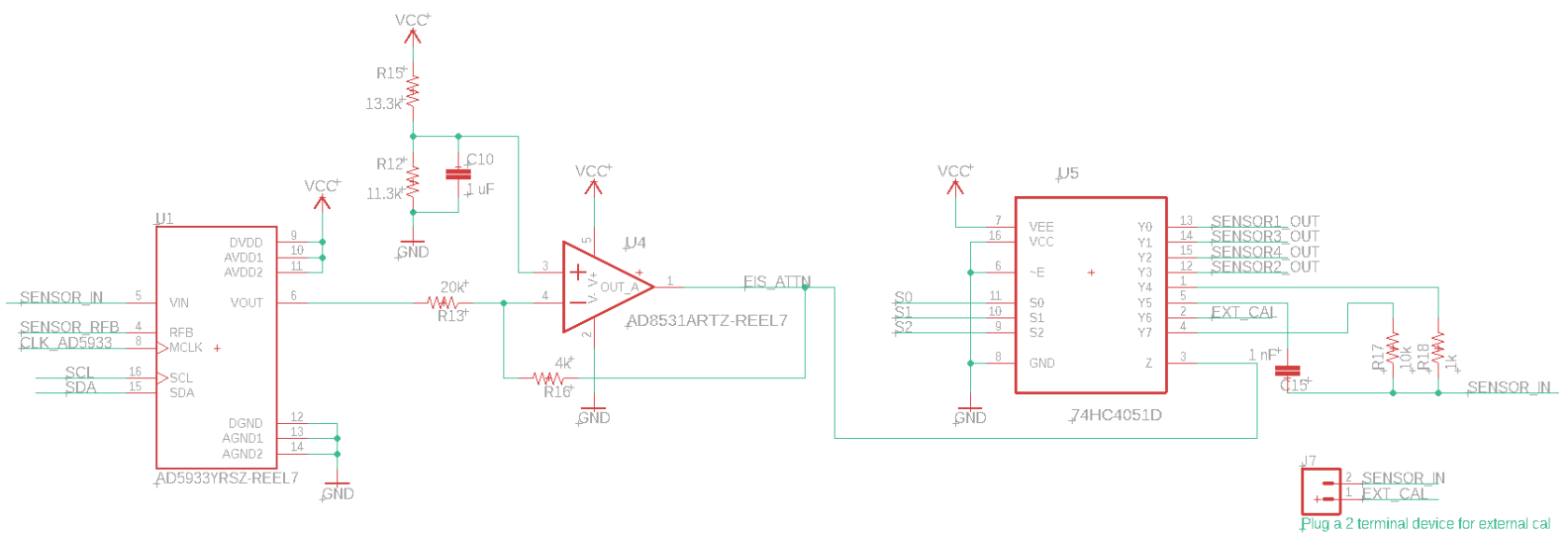

Figure 42 - EIS analog front end

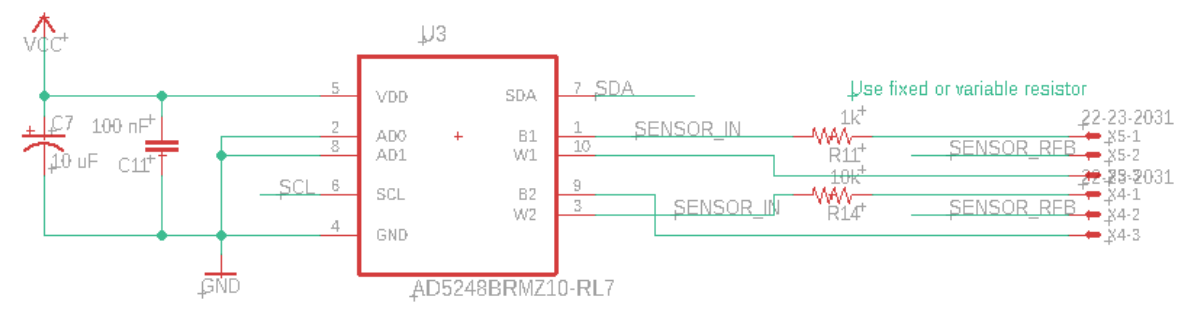

Figure 43 - EIS First Stage Gain 


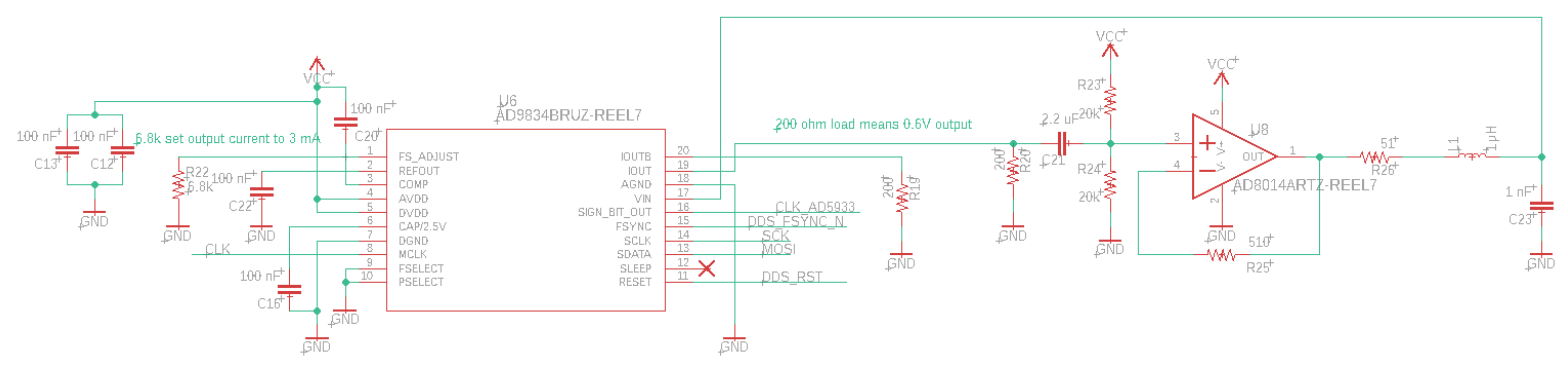

Figure 44 - Clock Generator

The DAC output is connected to a second order low pass $\mathrm{RC}$ filter set to $100 \mathrm{kHz}$, as any high frequencies components when a voltage step occurs should be filtered out. This signal passes to a non-inverting amplifier with has a gain of $1+\mathrm{R} 2 / \mathrm{R} 1$, and using standard $1 \%$ resistor values, setting R2 to $6.8 \mathrm{k} \Omega$ and $\mathrm{R} 1$ to $33 \mathrm{k} \Omega$ will provide a gain of $1.2 \mathrm{~V} / \mathrm{V}$. To improve the common mode rejection ratio of the current sensor, an additional 20 -ohm resistor is added to balance the inputs. The signal is then amplified and filtered using a 100 $\mathrm{kHz}$ low pass filter. 


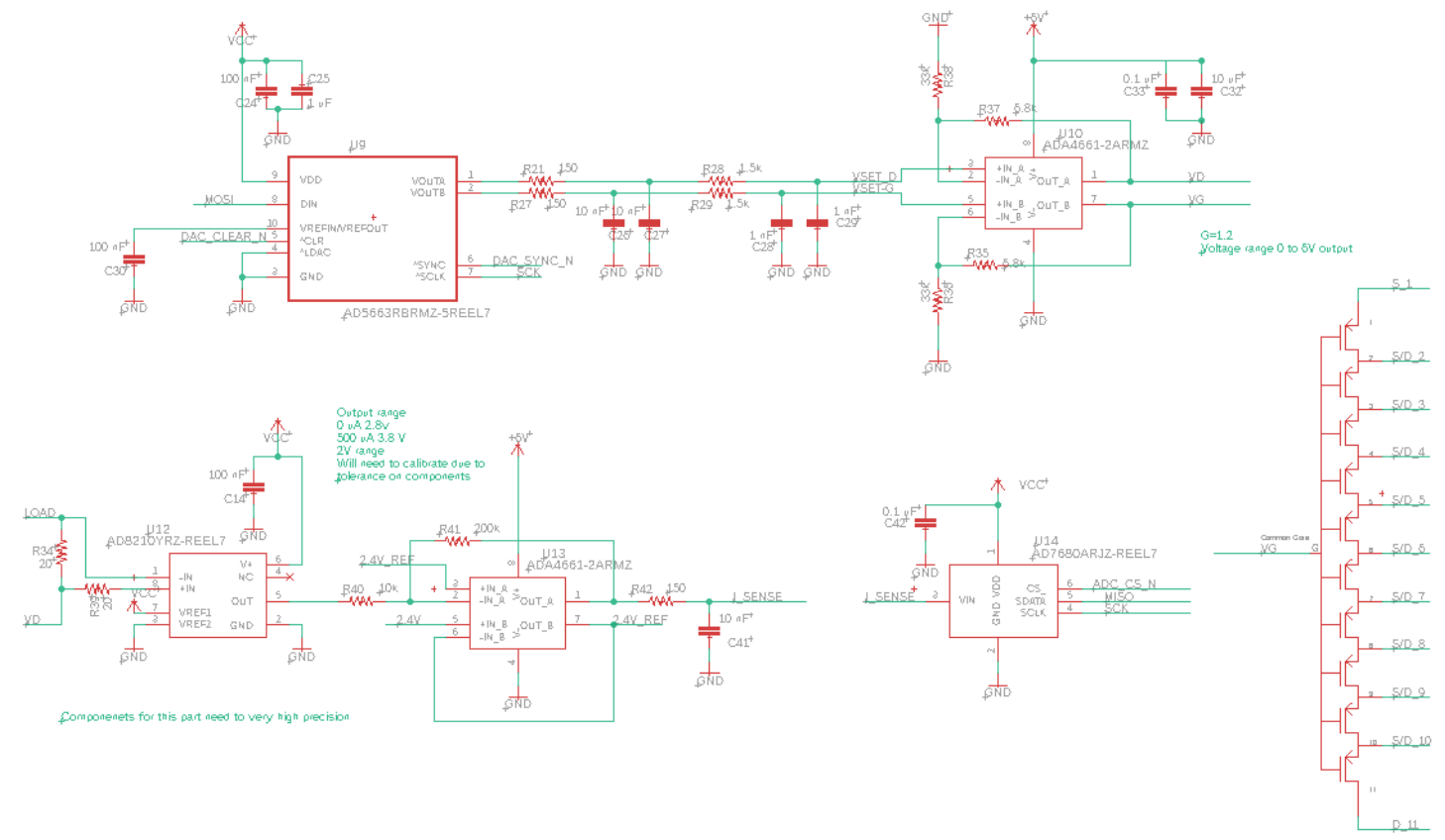

Figure 45 - PMOS Sensor Readout

In order to remain a portable low footprint device, the PCB size was set to $2.9 \times 3.5$ inches. Due to large number of components and the limitation on size, high-density manual routing was required to fit all the necessary components on the board. To help keep costs low a two-layer PCB was used, with 8 mil traces used for most signals and 10 mil traces used for all power and clock signals. Both layers have ground pours to reduce resistance to ground and help reduce noise. Another copper pour is used to connect all the four IDE return signals together to ensure they have equal resistance, and the same calibration can be used for all the IDEs. The two sensors were placed close together to keep microfluidic channel inputs and outputs on the same side of the board. The two sensors are packaged directly to the board by using epoxy to bond the substrate directly to the PCB. The microfluidic channels should already be bonded onto the sensors. The sensors are then wire bonded to the bonding pads on the $\mathrm{PCB}$, and epoxy is used to 
encapsulate the sensors. The PDMS microchannel used should be able to withstand the wire bonding temperatures of approximately $80^{\circ} \mathrm{C}$.

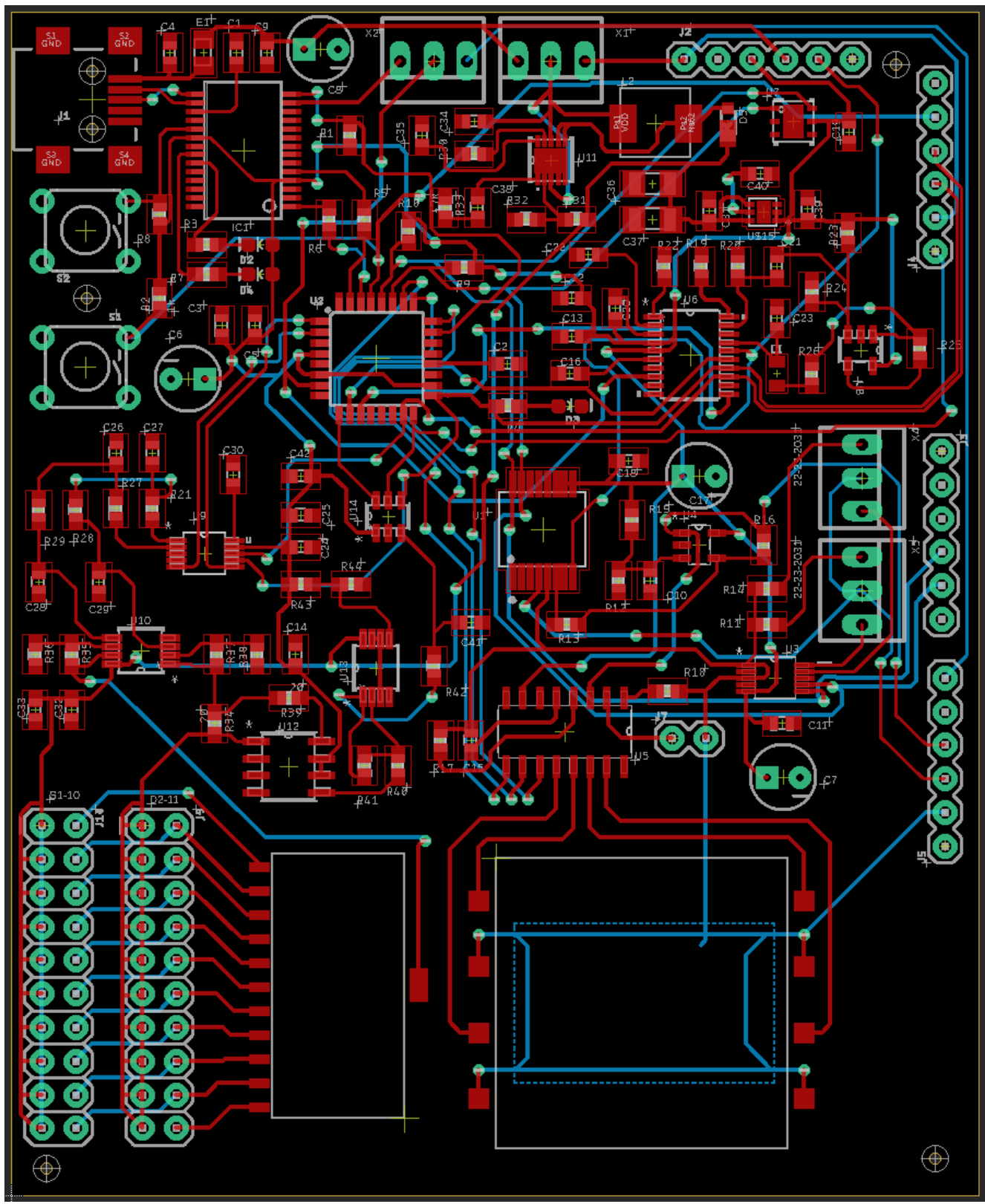

Figure 46 - Board Layout (without copper pours) 


\section{Bibliography}

[1] J. Hunt, "Exhaled breath condensate: an overview," Immunology and allergy clinics of North America, vol. 27, p. 587-596, 2007.

[2] P. Montuschi, C. T. Giovanni, P. Paredi, P. Pantelidis, R. M. Du Bois, S. A. Kharitonov and P. J. Barnes, "8-Isoprostane as a biomarker of oxidative stress in interstitial lung diseases," American journal of respiratory and critical care medicine, vol. 158, p. 1524-1527, 1998.

[3] I.-D. Kim, S.-J. Choi, S.-J. Kim and J.-S. Jang, "Exhaled breath sensors," Smart Sensors for Health and Environment Monitoring, p. 19-49, 2015.

[4] S. El-Akaad, M. A. Mohamed, N. S. Abdelwahab, E. A. Abdelaleem, S. De Saeger and N. Beloglazova, "Capacitive sensor based on molecularly imprinted polymers for detection of the insecticide imidacloprid in water," Scientific reports, vol. 10, p. 1-10, 2020.

[5] J. J. BelBruno, "Molecularly imprinted polymers," Chemical reviews, vol. 119, p. 94-119, 2018.

[6] O. Brüggemann, K. Haupt, L. Ye, E. Yilmaz and K. Mosbach, "New configurations and applications of molecularly imprinted polymers," Journal of Chromatography A, vol. 889, p. 15-24, 2000.

[7] H. Huzein Fahmi, S. Nurul Maisyarah, A. Yeon, M. Shakaff, W. Yufridin, H. Uda, Z. Ammar, A. G. Supri, M. Noor and P. Ahmad, "Highly selective molecular 
imprinted polymer (MIP) based sensor array using interdigitated electrode (IDE) platform for detection of mango ripeness," 2013.

[8] A.-M. Poller, E. Spieker, P. A. Lieberzeit and C. Preininger, "Surface imprints: Advantageous application of ready2use materials for bacterial quartz-crystal microbalance sensors," ACS applied materials \& interfaces, vol. 9, p. 1129-1135, 2017.

[9] M. V. Polyakov, "Adsorption properties and structure of silica gel," Zhur Fiz Khim, vol. 2, p. 799-805, 1931.

[10] L. Pauling, "Tailor-made compounds predicted by Pauling," Chemical \& Engineering News, 1949.

[11] F. H. Dickey, "The preparation of specific adsorbents," Proceedings of the National Academy of Sciences of the United States of America, vol. 35, p. 227, 1949.

[12] E. I. Klabunovskii, L. M. Volkova and A. E. Agronomov, "A new method of preparation of stereospecific silica gels," Izv. Akad. Nauk SSSR, Ser. Khim, vol. 2101, 1961.

[13] G. Wulff, "Molecular imprinting," Annals of the New York Academy of Sciences, vol. 434, p. 327-333, 1984.

[14] J. W. Lowdon, H. Diliën, P. Singla, M. Peeters, T. J. Cleij, B. van Grinsven and K. Eersels, "MIPs for commercial application in low-cost sensors and assays-An overview of the current status quo," Sensors and Actuators B: Chemical, p. 128973, 2020. 
[15] S. Akgönüllü, H. Yavuz and A. Denizli, "SPR nanosensor based on molecularly imprinted polymer film with gold nanoparticles for sensitive detection of aflatoxin B1," Talanta, vol. 219, p. 121219, 2020.

[16] Y. Liu, T. Shen, L. Hu, H. Gong, C. Chen, X. Chen and C. Cai, "Development of a thermosensitive molecularly imprinted polymer resonance light scattering sensor for rapid and highly selective detection of hepatitis A virus in vitro," Sensors and Actuators B: Chemical, vol. 253, p. 1188-1193, 2017.

[17] S. K. Jha and K. Hayashi, "Polyacrylic acid polymer and aldehydes template molecule based MIPs coated QCM sensors for detection of pattern aldehydes in body odor," Sensors and Actuators B: Chemical, vol. 206, p. 471-487, 2015.

[18] A. Tretjakov, V. Syritski, J. Reut, R. Boroznjak and A. Opik, "Molecularly imprinted polymer film interfaced with Surface Acoustic Wave technology as a sensing platform for label-free protein detection," Analytica chimica acta, vol. 902, pp. 182--188, 2016.

[19] J. S. Lee, J. Oh, S. G. Kim and J. Jang, "Highly Sensitive and Selective FieldEffect-Transistor NonEnzyme Dopamine Sensors Based on Pt/Conducting Polymer Hybrid Nanoparticles," Small, vol. 11, p. 2399-2406, 2015.

[20] F. Canfarotta, J. Czulak, A. Guerreiro, A. G. Cruz, S. Piletsky, G. E. Bergdahl, M. Hedström and B. Mattiasson, "A novel capacitive sensor based on molecularly imprinted nanoparticles as recognition elements," Biosensors and Bioelectronics, vol. 120, p. 108-114, 2018. 
[21] P. Singh, "SPR biosensors: historical perspectives and current challenges," Sensors and actuators B: Chemical, vol. 229, p. 110-130, 2016.

[22] P. K. Jain, X. Huang, I. H. El-Sayed and M. A. El-Sayed, "Review of some interesting surface plasmon resonance-enhanced properties of noble metal nanoparticles and their applications to biosystems," Plasmonics, vol. 2, p. 107$118,2007$.

[23] A. Abbas, M. J. Linman and Q. Cheng, "New trends in instrumental design for surface plasmon resonance-based biosensors," Biosensors and Bioelectronics, vol. 26, p. 1815-1824, 2011.

[24] M. Lotierzo, O. Y. F. Henry, S. Piletsky, I. Tothill, D. Cullen, M. Kania, B. Hock and A. P. F. Turner, "Surface plasmon resonance sensor for domoic acid based on grafted imprinted polymer," Biosensors and Bioelectronics, vol. 20, p. 145-152, 2004.

[25] J. Ling, C. Z. Huang, Y. F. Li, Y. F. Long and Q. G. Liao, "Recent developments of the resonance light scattering technique: technical evolution, new probes and applications," Applied Spectroscopy Reviews, vol. 42, p. 177-201, 2007.

[26] C. Z. Huang and Y. F. Li, "Resonance light scattering technique used for biochemical and pharmaceutical analysis," Analytica chimica acta, vol. 500, p. 105-117, 2003.

[27] F. Zhang, L. Luo, H. Gong, C. Chen and C. Cai, "A magnetic molecularly imprinted optical chemical sensor for specific recognition of trace quantities of virus," RSC advances, vol. 8, p. 32262-32268, 2018. 
[28] Y. Zhang, J. Zhang and Q. Liu, "Gas sensors based on molecular imprinting technology," Sensors, vol. 17, p. 1567, 2017.

[29] A. Alassi, M. Benammar and D. Brett, "Quartz crystal microbalance electronic interfacing systems: A review," Sensors, vol. 17, p. 2799, 2017.

[30] G. Sauerbrey, "Verwendung von Schwingquarzen zur Wagung dunner Schichten und zur Mikrowagung," Zeitschrift fur physik, vol. 155, no. 2, pp. 206--222, 1959.

[31] S. Emir Diltemiz, R. Keçili, A. Ersöz and R. Say, "Molecular imprinting technology in quartz crystal microbalance (QCM) sensors," Sensors, vol. 17, p. 454, 2017.

[32] M. Matsuguchi and T. Uno, "Molecular imprinting strategy for solvent molecules and its application for QCM-based VOC vapor sensing," Sensors and Actuators B: Chemical, vol. 113, p. 94-99, 2006.

[33] G. Mustafa and P. A. Lieberzeit, "Molecularly imprinted polymer-Ag $2 \mathrm{~S}$ nanoparticle composites for sensing volatile organics," RSC Advances, vol. 4, p. 12723-12728, 2014.

[34] K. Länge, "Bulk and surface acoustic wave sensor arrays for multi-analyte detection: A review," Sensors, vol. 19, p. 5382, 2019.

[35] K. Länge, B. E. Rapp and M. Rapp, "Surface acoustic wave biosensors: a review," Analytical and bioanalytical chemistry, vol. 391, p. 1509-1519, 2008.

[36] K. Kabir, S. J. Ippolito, Y. M. Sabri, C. J. Harrison, G. Matthews and S. K. Bhargava, "A comparison of Surface Acoustic Wave (SAW) and Quartz Crystal 
Microbalance (QCM) based sensors for portable, online mercury vapour sensing," Chemeca 2014: Processing excellence; Powering our future, p. 1402, 2014.

[37] A. Kidakova, R. Boroznjak, J. Reut, A. Öpik, M. Saarma and V. Syritski, "Molecularly imprinted polymer-based SAW sensor for label-free detection of cerebral dopamine neurotrophic factor protein," Sensors and Actuators B: Chemical, vol. 308, p. 127708, 2020.

[38] E. Hedborg, F. Winquist, I. Lundström, L. I. Andersson and K. Mosbach, "Some studies of molecularly-imprinted polymer membranes in combination with fieldeffect devices," Sensors and actuators A: Physical, vol. 37, p. 796-799, 1993.

[39] X. Hu and W. Yang, "Planar capacitive sensors-designs and applications," Sensor Review, 2010.

[40] E. De Rycke, O. Leman, P. Dubruel, M. Hedström, M. Völker, N. Beloglazova and S. De Saeger, "Novel multiplex capacitive sensor based on molecularly imprinted polymers: A promising tool for tracing specific amphetamine synthesis markers in sewage water," Biosensors and Bioelectronics, vol. 178, p. 113006, 2021.

[41] R. D. Crapnell, A. Hudson, C. W. Foster, K. Eersels, B. v. Grinsven, T. J. Cleij, C. E. Banks and M. Peeters, "Recent advances in electrosynthesized molecularly imprinted polymer sensing platforms for bioanalyte detection," Sensors, vol. 19, p. $1204,2019$.

[42] P. Montuschi, J. V. Collins, G. Ciabattoni, N. Lazzeri, M. Corradi, S. A. Kharitonov and P. J. Barnes, "Exhaled 8-isoprostane as an in vivo biomarker of 
lung oxidative stress in patients with COPD and healthy smokers," American journal of respiratory and critical care medicine, vol. 162, p. 1175-1177, 2000.

[43] P. Montuschi, P. J. Barnes and G. Ciabattoni, "Measurement of 8-isoprostane in exhaled breath condensate," in Advanced Protocols in Oxidative Stress II, Springer, 2010, p. 73-84.

[44] R. Selvaraj, N. J. Vasa, S. M. Nagendra and B. Mizaikoff, "Advances in midinfrared spectroscopy-based sensing techniques for exhaled breath diagnostics," Molecules, vol. 25, p. 2227, 2020.

[45] S.-W. Chiu and K.-T. Tang, "Towards a chemiresistive sensor-integrated electronic nose: a review," Sensors, vol. 13, no. 10, pp. 14214-14247, 2013.

[46] F.-L. Meng, Z. Guo and X.-J. Huang, "Graphene-based hybrids for chemiresistive gas sensors," TrAC Trends in Analytical Chemistry, vol. 68, p. 37-47, 2015.

[47] I. Amor, B. Gamero, S. Bebe and R. Prakash, "Hydrothermally Fluorinated Graphene Oxide Chemiresistive Sensor for Detecting NH3 and Acetone under Atmospheric Conditions.," in BIODEVICES, 2021.

[48] S. Kim, K.-H. Lee, J.-Y. Lee, K.-K. Kim, Y.-H. Choa and J.-H. Lim, "SingleWalled Carbon Nanotube-Based Chemi-Capacitive Sensor for Hexane and Ammonia," Electronic Materials Letters, vol. 15, no. 6, pp. 712-719, 2019.

[49] A. Rico-Yuste and S. Carrasco, "Molecularly imprinted polymer-based hybrid materials for the development of optical sensors," Polymers, vol. 11, p. 1173, 2019. 
[50] N. W. Turner, C. W. Jeans, K. R. Brain, C. J. Allender, V. Hlady and D. W. Britt, "From 3D to 2D: a review of the molecular imprinting of proteins," Biotechnology progress, vol. 22, p. 1474-1489, 2006.

[51] D. Refaat, M. G. Aggour, A. A. Farghali, R. Mahajan, J. G. Wiklander, I. A. Nicholls and S. A. Piletsky, "Strategies for molecular imprinting and the evolution of MIP nanoparticles as plastic antibodies-Synthesis and applications," International journal of molecular sciences, vol. 20, p. 6304, 2019.

[52] Z. O. Uygun, H. D. E. Uygun, N. Ermiş and E. Canbay, "Molecularly imprinted sensors-New sensing technologies," Biosensors-Micro and Nanoscale Applications, p. 85-108, 2015.

[53] M. Menger, A. Yarman, J. Erdőssy, H. B. Yildiz, R. E. Gyurcsányi and F. W. Scheller, "MIPs and aptamers for recognition of proteins in biomimetic sensing," Biosensors, vol. 6, p. 35, 2016.

[54] T. Kobayashi, T. Fukaya, M. Abe and N. Fujii, "Phase inversion molecular imprinting by using template copolymers for high substrate recognition," Langmuir, vol. 18, p. 2866-2872, 2002.

[55] H. Lalo, C. Ayela, E. Dague, C. Vieu and K. Haupt, "Nanopatterning molecularly imprinted polymers by soft lithography: a hierarchical approach," Lab on a Chip, vol. 10, p. 1316-1318, 2010.

[56] K. Haupt, P. X. Medina Rangel and B. T. S. Bui, "Molecularly imprinted polymers: Antibody mimics for bioimaging and therapy," Chemical Reviews, vol. 120, p. 9554-9582, 2020. 
[57] K. Smolinska-Kempisty, A. Guerreiro, F. Canfarotta, C. Cáceres, M. J. Whitcombe and S. Piletsky, "A comparison of the performance of molecularly imprinted polymer nanoparticles for small molecule targets and antibodies in the ELISA format," Scientific reports, vol. 6, p. 1-7, 2016.

[58] S. N. Hashim, R. I. Boysen, L. J. Schwarz, B. Danylec and M. T. Hearn, "A comparison of covalent and non-covalent imprinting strategies for the synthesis of stigmasterol imprinted polymers," Journal of Chromatography A, vol. 1359, pp. $35-43,2014$.

[59] Poly(vinyl alcohol), N-methyl-4(4'-formylstyryl)pyridinium methosulfate acetal, Polysciences.

[60] 8-iso Prostaglandin F2 $\alpha$ (CAS 27415-26-5).

[61] E. Team, PDMS: a review on polydimethylsiloxane in microfluidics, Elvesys, 2021.

[62] J. Gu, J. Huang, G. Chen, L. Hou, J. Zhang, X. Zhang, X. Yang, L. Guan, X. Jiang and H. Liu, "Multifunctional Poly (vinyl alcohol) Nanocomposite Organohydrogel for Flexible Strain and Temperature Sensor," ACS Applied Materials \& Interfaces, vol. 12 , p. $40815-40827,2020$.

[63] I. A. Latif, H. M. Abdullah and M. H. Saleem, "Electrical and swelling study of different prepared hydrogel," Am J Polym Sci, vol. 6, p. 50-57, 2016.

[64] K. S. Chan, H. B. Senin and I. Naimah, "Structural and mechanical properties of polyvinyl alcohol (PVA) thin film," in AIP Conference Proceedings, 2009. 
[65] Z. Li, W. Xu, X. Wang, W. Jiang, X. Ma, F. Wang, C. Zhang and C. Ren, "Fabrication of PVA/PAAm IPN hydrogel with high adhesion and enhanced mechanical properties for body sensors and antibacterial activity," European Polymer Journal, vol. 146, p. 110253, 2021.

[66] Z. Li, D. Wang, H. Bai, S. Zhang, P. Ma and W. Dong, "Photo-Crosslinking Strategy Constructs Adhesive, Superabsorbent, and Tough PVA-Based Hydrogel through Controlling the Balance of Cohesion and Adhesion," Macromolecular Materials and Engineering, vol. 305, p. 1900623, 2020.

[67] K. Ichimura, S. Iwata, S. Mochizuki, M. Ohmi and D. Adachi, "Revisit to the photocrosslinking behavior of PVA-SbQ as a water-soluble photopolymer with anomalously low contents of quaterized stilbazol side chains," Journal of Polymer Science Part A: Polymer Chemistry, vol. 50, p. 4094-4102, 2012.

[68] S. Fujisawa, M. Yamamoto, D. Kashiwai, P. Azari, Y. Y. Khaw, S. N. Gan and S. Takahara, "Formation Behavior of Polyiodine Complex in Photocrosslinked Polyvinyl Alcohol Fiber Spun by Electrospinning Method," Journal of Photopolymer Science and Technology, vol. 31, no. 4, pp. 569-574, 2018.

[69] K. Raj M and S. Chakraborty, "PDMS microfluidics: A mini review," Journal of Applied Polymer Science, vol. 137, p. 48958, 2020.

[70] A. Victor, J. E. Ribeiro and F. F. Araújo, "Study of PDMS characterization and its applications in biomedicine: A review," Journal of Mechanical Engineering and Biomechanics, vol. 4, p. 1-9, 2019. 
[71] S. Rana, R. H. Page and C. J. McNeil, "Impedance spectra analysis to characterize interdigitated electrodes as electrochemical sensors," Electrochimica acta, vol. 56, p. 8559-8563, 2011.

[72] B.-Y. Chang and S.-M. Park, "Electrochemical impedance spectroscopy," Annual Review of Analytical Chemistry, vol. 3, p. 207-229, 2010.

[73] G. J. Brug, A. L. G. van den Eeden, M. Sluyters-Rehbach and J. H. Sluyters, "The analysis of electrode impedances complicated by the presence of a constant phase element," Journal of electroanalytical chemistry and interfacial electrochemistry, vol. 176, p. $275-295,1984$.

[74] A. Allagui, A. S. Elwakil and C. Psychalinos, "Decoupling the magnitude and phase in a constant phase element," Journal of Electroanalytical Chemistry, vol. 888, p. 115153, 2021.

[75] P. M. Biesheuvel, S. Porada and J. E. Dykstra, "The difference between Faradaic and non-Faradaic electrode processes," arXiv preprint arXiv:1809.02930, 2018.

[76] G. Instruments, "Basics of electrochemical impedance spectroscopy," G. Instruments, Complex impedance in Corrosion, p. 1-30, 2007.

[77] R. Nangia, N. K. Shukla and A. Sharma, "Preparation, structural and dielectric properties of solution grown polyvinyl alcohol (PVA) film," in IOP Conference Series: Materials Science and Engineering, 2017.

[78] Y. K. Yeow, Z. Abbas, K. Khalid and M. Z. A. Rahman, "Improved dielectric model for polyvinyl alcohol-water hydrogel at microwave frequencies," American Journal of Applied Sciences, vol. 7, p. 270, 2010. 
[79] M. Ibrahim, J. Claudel, D. Kourtiche and M. Nadi, "Geometric parameters optimization of planar interdigitated electrodes for bioimpedance spectroscopy," Journal of Electrical Bioimpedance, vol. 4, p. 13-22, 2013.

[80] W. Olthuis, W. Streekstra and P. Bergveld, "Theoretical and experimental determination of cell constants of planar-interdigitated electrolyte conductivity sensors," Sensors and Actuators B: Chemical, vol. 24, p. 252-256, 1995.

[81] J. Oberländer, Z. B. Jildeh, P. Kirchner, L. Wendeler, A. Bromm, H. Iken, P. Wagner, M. Keusgen and M. J. Schöning, "Study of interdigitated electrode arrays using experiments and finite element models for the evaluation of sterilization processes," Sensors, vol. 15, p. 26115-26127, 2015.

[82] M. Czerska, K. Mikolajewska, M. Zielinski, J. Gromadzinska and W. Wasowicz, "Today’s oxidative stress markers," 2015.

[83] R. Massey, R. Amache, S. Bebe and R. Prakash, "A Comprehensive Modelling Approach for Bio-EDLC systems," in 2020 IEEE Sensors, 2020.

[84] "megaAVR® Data Sheet," 2020.

[85] "FT232R USB UART IC," 2021.

[86] "650 kHz/1.3 MHz, 4 A, Step-Up, PWM, DC-to-DC Switching Converter," 2014.

[87] "20 V, 200 mA, Low Noise, CMOS LDO Linear Regulator," 2020.

[88] "1 MSPS, 12-Bit Impedance Converter, Network Analyzer," 2017.

[89] "8-channel analog multiplexer/demultiplexer," 2017.

[90] "Dual, 256-Position, I2C-Compatible Digital Potentiometers," 2016.

[91] "20 mW Power, 2.3 V to 5.5 V, 75 MHz Complete DDS," 2014. 
[92] "2.7 V to $5.5 \mathrm{~V}, 250 \mu \mathrm{A}$, Rail-to-Rail Output, Dual 16-Bit nanoDAC," 2016.

[93] "High-Voltage, Bidirectional Current Shunt Monitor," 2020.

[94] "3 mW, 100 kSPS, 16-Bit ADC in 6-Lead SOT-23," 2011.

[95] E. Sanchez-Tirado, A. Gonzalez-Cortes, M. Yudasaka, S. Iijima, F. Langa, P. Yanez-Sedeno and J. d Pingarron, "Electrochemical immunosensor for the determination of 8-isoprostane aging biomarker using carbon nanohorns-modified disposable electrodes," Journal of Electroanalytical Chemistry, vol. 793, pp. 197$202,2017$.

[96] T. J. van't Erve, F. B. Lih, M. B. Kadiiska, L. J. Deterding, T. E. Eling and R. P. Mason, "Reinterpreting the best biomarker of oxidative stress: The 8-isoPGF $2 \alpha /$ PGF $2 \alpha$ ratio distinguishes chemical from enzymatic lipid peroxidation," Free Radical Biology and Medicine, vol. 83, p. 245-251, 2015.

[97] N. Zhang, N. Zhang, Y. Xu, Z. Li, C. Yan, K. Mei, M. Ding, S. Ding, P. Guan, L. Qian and others, "Molecularly imprinted materials for selective biological recognition," Macromolecular rapid communications, vol. 40, p. 1900096, 2019.

[98] S. Zanconato, S. Carraro, M. Corradi, R. Alinovi, M. F. Pasquale, G. Piacentini, F. Zacchello and E. Baraldi, "Leukotrienes and 8-isoprostane in exhaled breath condensate of children with stable and unstable asthma," Journal of Allergy and Clinical Immunology, vol. 113, p. 257-263, 2004.

[99] L. Wang, "Metal-organic frameworks for QCM-based gas sensors: A review," Sensors and Actuators A: Physical, vol. 307, p. 111984, 2020. 
[100] F. Usman, J. O. Dennis, A. Y. Ahmed, F. Meriaudeau, O. B. Ayodele and A. A. S. Rabih, "A review of biosensors for non-invasive diabetes monitoring and screening in human exhaled breath," IEEE Access, vol. 7, p. 5963-5974, 2018.

[101] T. J. Sherbow, G. M. Kuhl, G. A. Lindquist, J. D. Levine, M. D. Pluth, D. W. Johnson and S. A. Fontenot, "Hydrosulfide-selective ChemFETs for aqueous H2S/HS- measurement," Sensing and Bio-Sensing Research, vol. 31, p. 100394, 2021.

[102] S. b. Granite, Fluorescence Measurements \& Instrumentation: Spectrofluorometer, 2020.

[103] S. Gaggiotti, F. Della Pelle, M. Mascini, A. Cichelli and D. Compagnone, "Peptides, DNA and MIPs in gas sensing. From the realization of the sensors to sample analysis," Sensors, vol. 20, p. 4433, 2020.

[104] A. Adumitrăchioaie, M. Tertiș, A. Cernat, R. Săndulescu and C. Cristea, "Electrochemical methods based on molecularly imprinted polymers for drug detection. A review," Int. J. Electrochem. Sci, vol. 13, p. 2556-2576, 2018. 